University of Louisville

ThinkIR: The University of Louisville's Institutional Repository

Electronic Theses and Dissertations

$12-2014$

\title{
Transient heat transfer between a magnetocaloric packed particle bed and stagnant interstitial fluid.
}

Michael G. Schroeder 1988-

University of Louisville

Follow this and additional works at: https://ir.library.louisville.edu/etd

Part of the Mechanical Engineering Commons

\section{Recommended Citation}

Schroeder, Michael G. 1988-, "Transient heat transfer between a magnetocaloric packed particle bed and stagnant interstitial fluid." (2014). Electronic Theses and Dissertations. Paper 1782.

https://doi.org/10.18297/etd/1782

This Master's Thesis is brought to you for free and open access by ThinkIR: The University of Louisville's Institutional Repository. It has been accepted for inclusion in Electronic Theses and Dissertations by an authorized administrator of ThinkIR: The University of Louisville's Institutional Repository. This title appears here courtesy of the author, who has retained all other copyrights. For more information, please contact thinkir@louisville.edu. 


\title{
TRANSIENT HEAT TRANSFER BETWEEN A MAGNETOCALORIC PACKED PARTICLE BED AND STAGNANT INTERSTITIAL FLUID
}

\author{
By \\ Michael G. Schroeder \\ B.S. University of Toledo
}

\begin{abstract}
A Thesis
Submitted to the Faculty of the

University of Louisville

J.B. Speed School of Engineering

in Partial Fulfillment of the Requirements

for the Degree of

Master of Science

Department of Mechanical Engineering

University of Louisville

Louisville, KY
\end{abstract}

December, 2014 

TRANSIENT HEAT TRANSFER BETWEEN A MAGNETOCALORIC PACKED PARTICLE BED AND STAGNANT INTERSTITIAL FLUID

\author{
By \\ Michael G. Schroeder \\ B.S. University of Toledo \\ A Thesis Approved On
}

November 25, 2014

by the Following Thesis Committee:

Ellen Brehob, Principal Advisor

Sam Park

R. Eric Berson 


\section{ACKNOWLEDGEMENTS}

I would like to express my thanks to my advisor, Ellen Brehob, for her continuous support throughout this project. You're insight and guidance have helped me grow as both a researcher and a writer. Additionally, I would like to thank Sam Park and R. Eric Berson for serving on my Thesis committee. You're questions and comments during the defense have been most valuable.

I would also like to thank those at GE who have been friends and mentors to me since I began working here. To my GE technical advisor, David Beers: You've been a tremendous mentor, allowing me to continuously improve myself as an engineer under your guidance. To the other GE Appliances magnetocaloric team members, Michael Benedict and Brian Schork, I thank you both sincerely for your daily support with creating and executing this project. 


\title{
ABSTRACT \\ TRANSIENT HEAT TRANSFER BETWEEN A MAGNETOCALORIC PACKED PARTICLE BED AND STAGNANT INTERSTITIAL FLUID
}

\author{
Michael G. Schroeder
}

November 25, 2014

In the field of magnetocaloric heat pumps, many system models have been made. What most of these models lack is heat transfer occurring while there is no fluid motion. It is the objective of this paper to analytically determine and experimentally verify heat transfer with no bulk fluid motion.

The addition of a pure dwell period to the typical magnetocaloric cycle was shown to have a positive performance impact in specific conditions. These conditions correspond to regenerators that are heat transfer rate limited in operation. The heat transfer mechanism for this particular case was shown to be pure conduction via a CFD study. Toroidal convective currents were present, but flow velocity was miniscule. Assuming pure conduction, an analytical model was created to calculate average heat transfer coefficient from geometric parameters and material properties. This heat transfer coefficient was rolled into an exponential coupled two mass model, which was validated via a time stepping model.

In order to experimentally validate the model, two experiments were performed. The first experiment yielded particle sphericity; which allows for an independent measurement of heat transfer area. The second experiment was performed in order to measure fluid temperature over time for various particle diameters, particle thermal conductivities, and fluid conductivities. The coupled two mass exponential model was fitted to the output of these tests to find $U A_{h x}$ (Heat transfer coefficient multiplied by heat transfer area). Heat transfer area was separately determined, allowing heat transfer coefficient $U$ to be separated from area and compared with the analytically determined values.

The analytical model required a scaling factor of 0.70 to be applied to calculate heat transfer rate, making the final correlation

$$
U=\frac{0.70}{\left(\frac{L_{C S}}{k_{S}}+\frac{L_{c f}}{k_{f}}\right)}=\frac{0.70}{\left(\frac{D_{p} \theta}{6 k_{S}}+\frac{D_{p} \varepsilon}{6(1-\varepsilon) k_{f}}\right)} .
$$


where $\theta$ is sphericity (0 to 1 ), $D_{p}$ is average particle diameter (Using sieve hole sizes used in sorting), $\varepsilon$ is void fraction in regenerator packing, $k_{s}$ is solid material conductivity, and $k_{f}$ is fluid material conductivity. 


\section{TABLE OF CONTENTS}

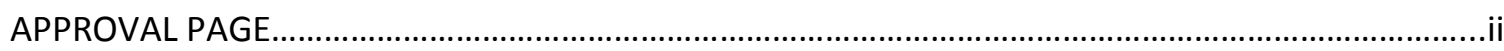

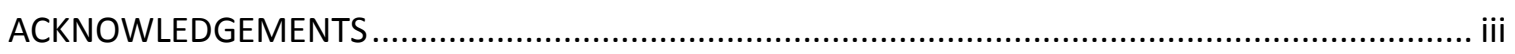

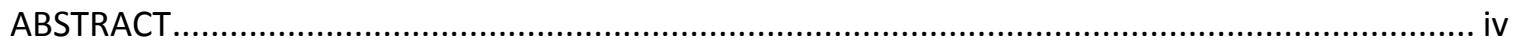

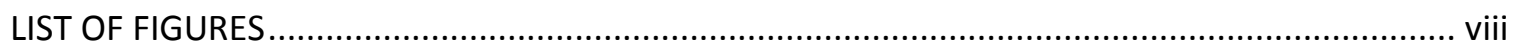

1 - INTRODUCTION TO HEAT PUMPS \& MAGNETOCALORIC TECHNOLOGY................................. 1

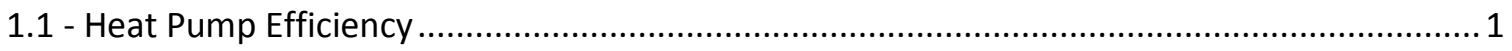

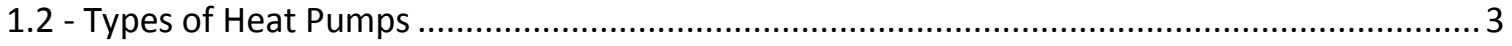

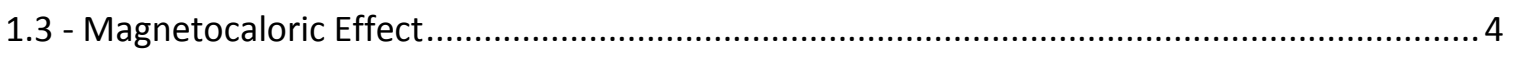

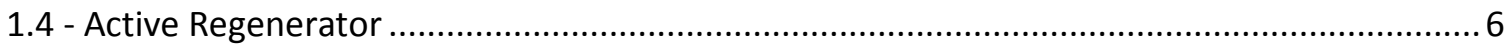

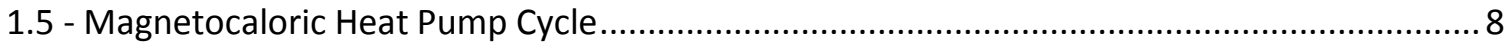

2 - PREVIOUS WORK RELATING TO HEAT TRANSFER DURING DWELL ...................................... 10

2.1 - Experimental Work on Magnetocaloric Devices.............................................................. 10

2.2 - Experimental Studies on Forced Convection .................................................................... 11

2.3 - Experimental Studies on Free Convection ........................................................................... 13

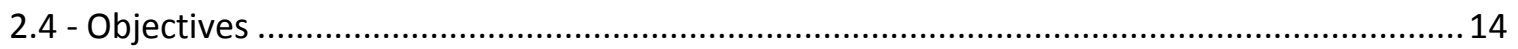

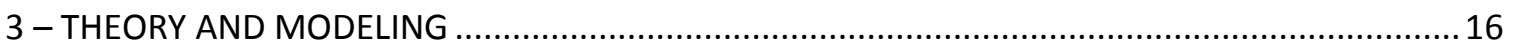

3.1 - Demonstrating the Usefulness of a Dwell Period ................................................................ 16

3.1.1 - Basic Two-Mass Time Stepping Model Setup .................................................................... 16

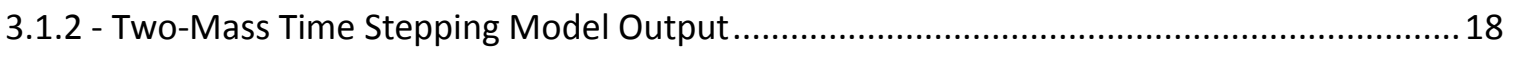

3.2 - CFD Study to Determine Dominant Heat Transfer Mechanism........................................... 21

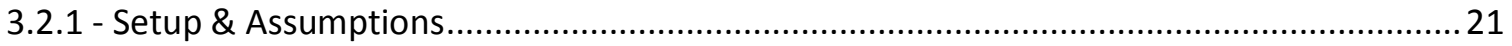

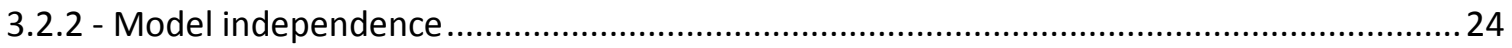

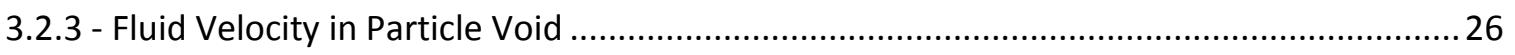

3.3 - Heat Transfer Model Development ….............................................................................. 29

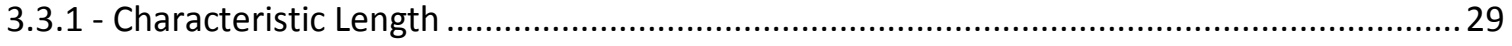

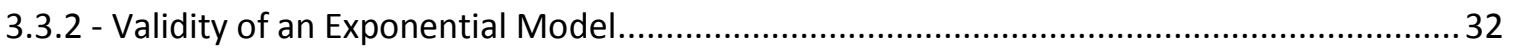


3.3.3 - Exponential Behavior of a Coupled Two Mass System

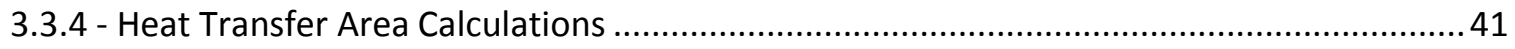

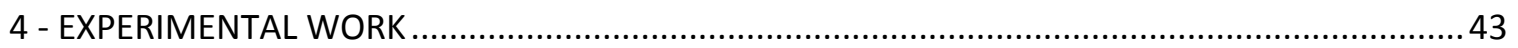

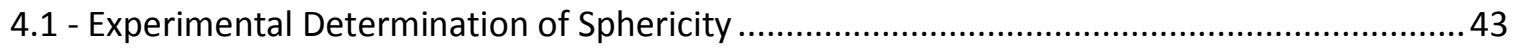

4.1.1 - Methodology for Determining Sphericity Experimentally ................................................ 43

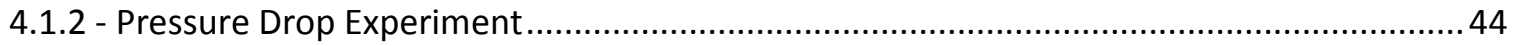

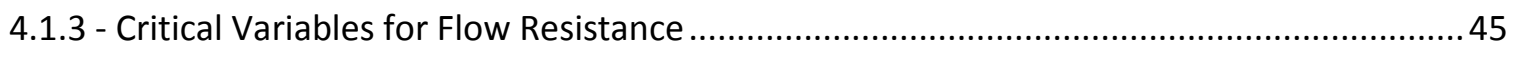

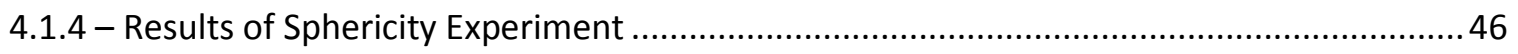

4.2 - Test Fixture Used for Heat Transfer Experiment …........................................................... 48

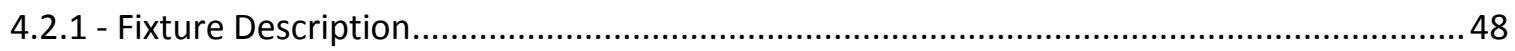

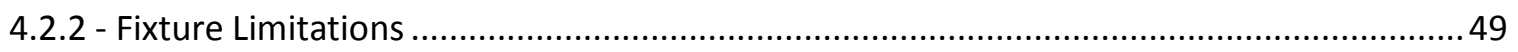

4.3 - Experiment to Determine Heat Transfer Rate during Dwell .................................................50

4.3.1 - Heat Transfer Design of Experiments ............................................................................. 50

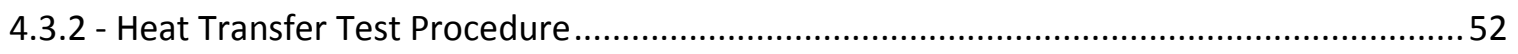

4.3.3 - Processing Raw Data to Yield Heat Transfer Coefficient ................................................55

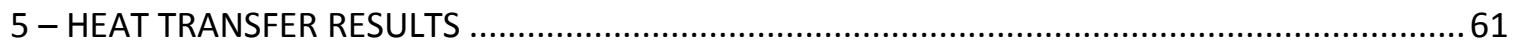

5.1 - Comparison between Measured and Predicted UA Values................................................61

5.2 - Creation of a Continuous Function Using a Flow Correlation.............................................. 62

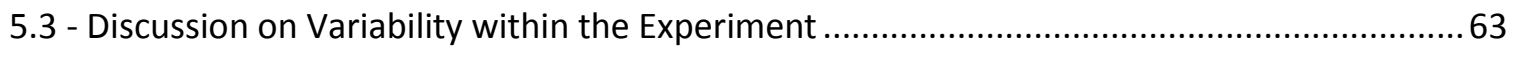

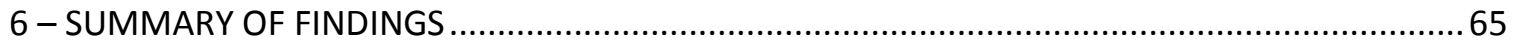

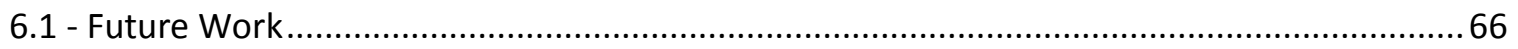

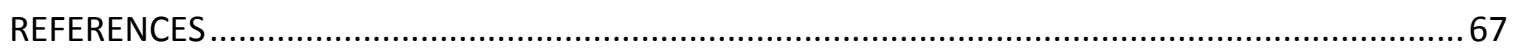

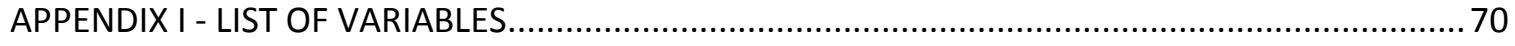

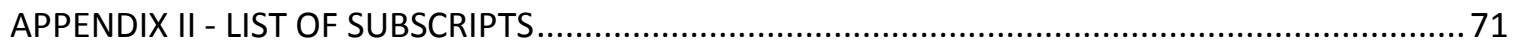

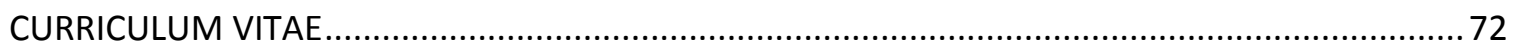




\section{LIST OF FIGURES}

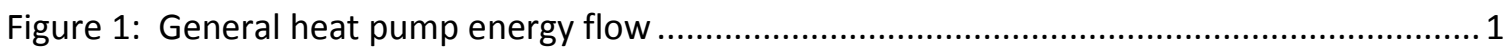

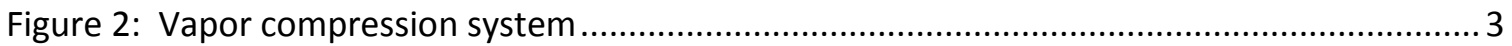

Figure 3: Magnetocaloric effect (Pecharsky and Gschneider, 1999, figure 1.1) ........................... 4

Figure 4: Isothermal entropy change (a) \& adiabatic temperature change (b) for Gd (Lee, 2004, figure 2)

Figure 5: Isothermal entropy change vs. temperature at 1.6 Tesla (Barcza et al, 2011, figure 6) .6 Figure 6: Photos of regenerators and particles ................................................................... 7

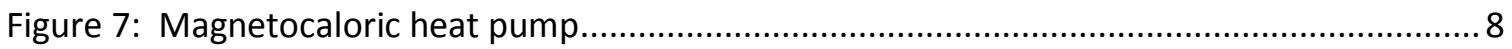

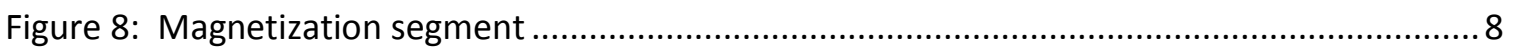

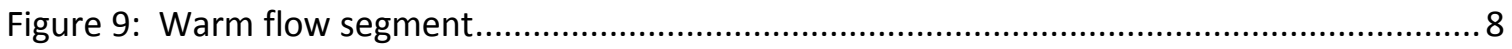

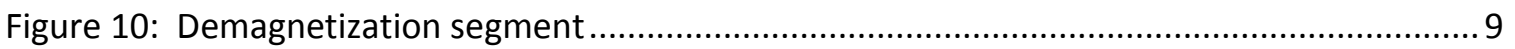

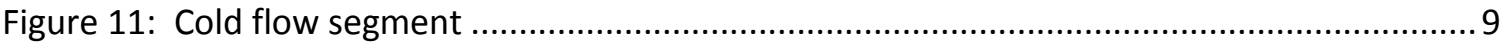

Figure 12: Experimental confidence ranges (Wakoa and Kagei, 1982, figure 3) .........................12

Figure 13: Half cycle state diagram using square-wave input ................................................... 16

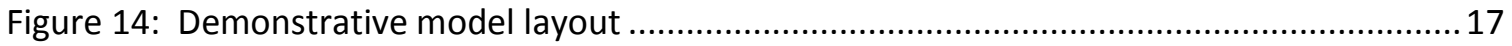

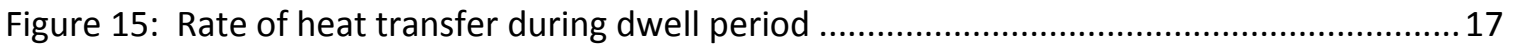

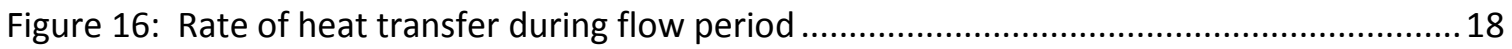

Figure 17: Outlet temperature profile with no dwell period..................................................... 18

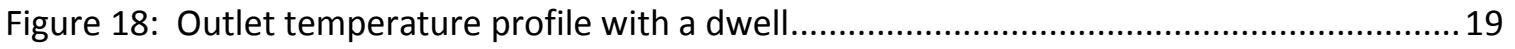

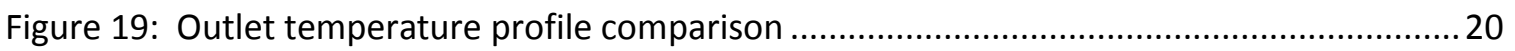

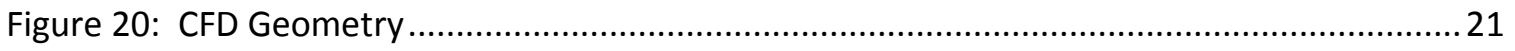

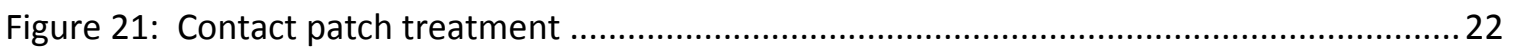

Figure 22: Cp vs. temperature curves for high and low magnetic states (Aliev et al., 2011) ....... 23

Figure 23: Model output vs. time step size for three particle diameters ..................................... 24

Figure 24: Model output vs. mesh size for three particle diameters ............................................ 25

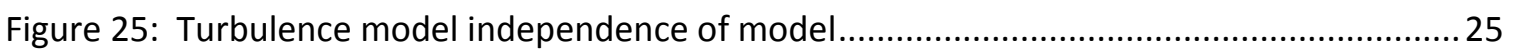

Figure 26: Average velocity vs. time for $300 \boldsymbol{\mu m}$ diameter particles ..........................................26

Figure 27: Average velocity vs. time for $900 \boldsymbol{\mu m}$ diameter particles ........................................27

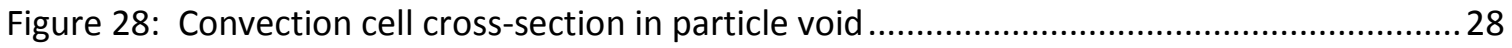

Figure 29: Particle diameter vs. Time to $1 \mathrm{~K}$ change in fluid temperature ...................................29

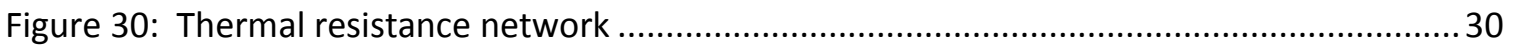

Figure 31: Spacial and temporal temperature distribution following a step change in MCM

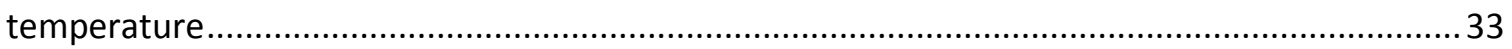

Figure 32: Exponential model of temperature change for two coupled masses...........................35 
Figure 33: Exponential model of temperature change for independent and coupled masses .... 36

Figure 34: Time constants for equivalent thermal and electrical networks...............................40

Figure 35: Exponential vs. time stepping model for fluid temperature change over time .......... 41

Figure 36: Diagram of pressure test apparatus .................................................................... 45

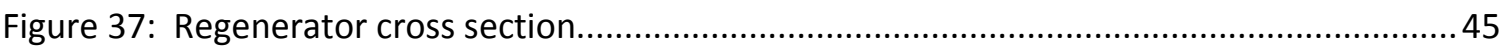

Figure 38: Regenerator pressure drop; predicted versus measured .......................................... 47

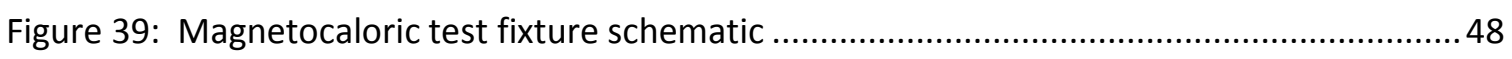

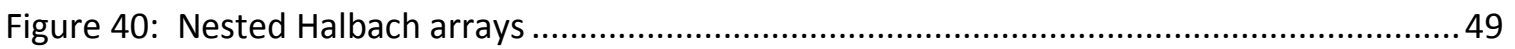

Figure 41: Tabulated working fluid properties ....................................................................... 51

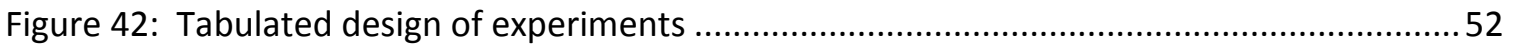

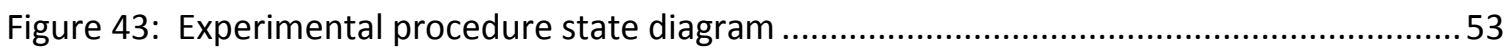

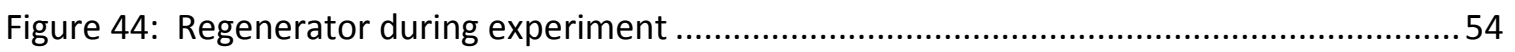

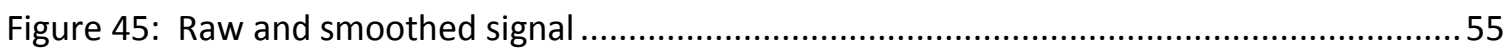

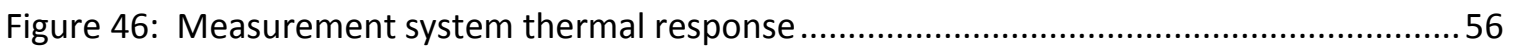

Figure 47: Finding fluid outlet temperature using system response .........................................57

Figure 48: Normalized fluid outlet temperature vs. test time ................................................... 58

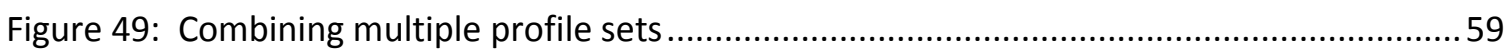

Figure 50: Average measured versus calculated specific UA values.........................................61 


\section{1 - INTRODUCTION TO HEAT PUMPS \& MAGNETOCALORIC TECHNOLOGY}

\section{1 - Heat Pump Efficiency}

A heat pump is a device which creates a temperature difference between a source and a sink. In doing so, thermal energy is moved from a low temperature to a high temperature. Historically, the main uses for the technology have been in cooling applications such as refrigeration and air conditioning. More recently, heat pumps have been used in heating applications as well. This is due to their improved heating efficiency over combustion and resistive heating. Water heaters and clothes dryers are among the newly reinvented heating applications. The flow of energy is typically like that shown in figure 1.

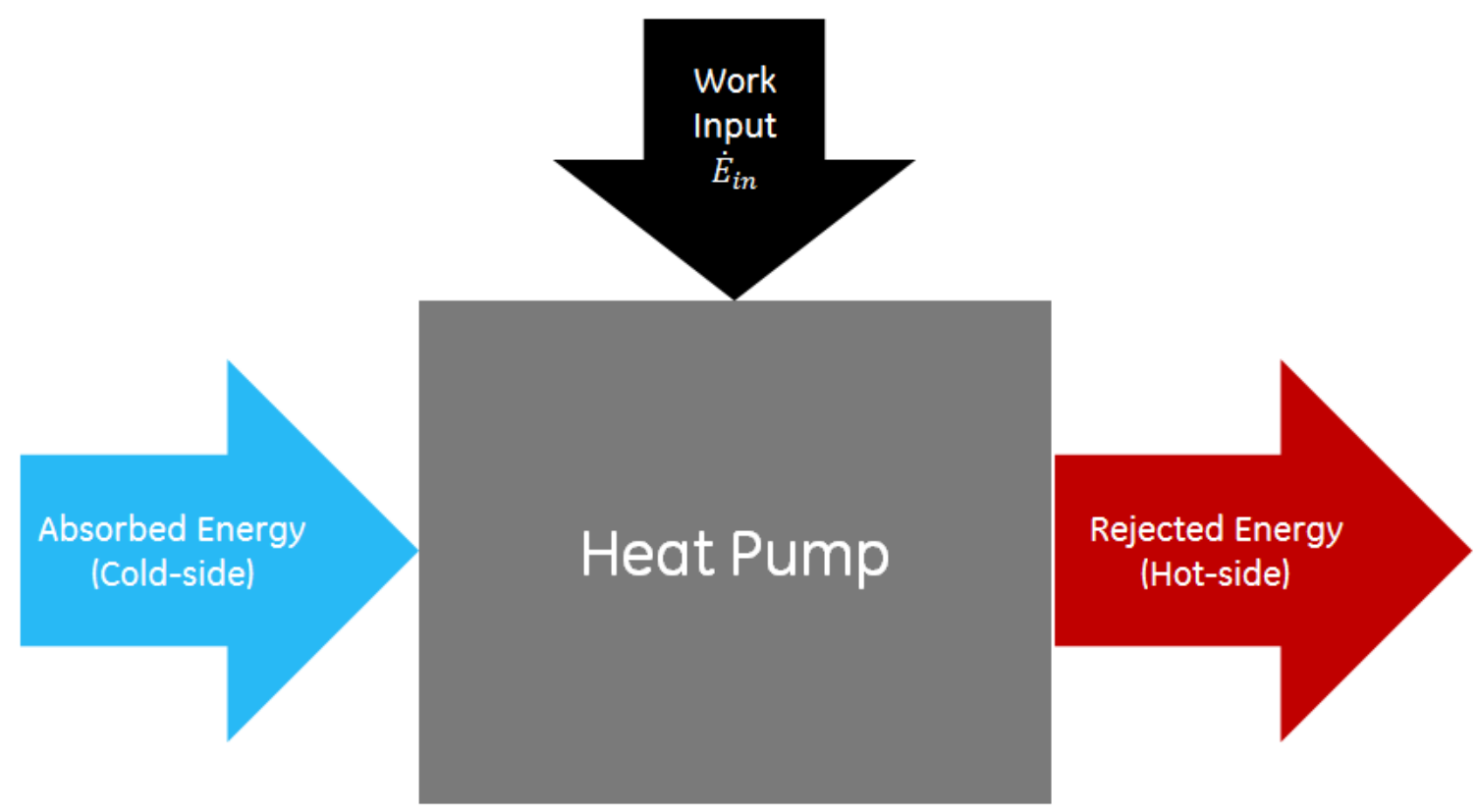

Figure 1: General heat pump energy flow

The terminology used to quantify machine efficiency is coefficient of performance, given by

$$
\text { COP }=\frac{\dot{E}_{\text {useful }}}{\dot{E}_{\text {in }}}
$$


where $\dot{E}_{\text {useful }}$ is useful energy flow (absorbed energy for cooling or rejected energy for heating) and $\dot{E}_{\text {in }}$ is energy that is put into the system to create the useful energy flow.

Energy rate out is generally desired heating or cooling capacity, and energy rate in is the work rate required to operate the device. As a trivial example, a resistive heating element water heater is capable of (and limited to) a COP of about one. This is because all electrical energy that is dissipated goes directly into heat. Conversely, a vapor compression water heater is capable of exceeding a COP of one. However, the issue with using COP as a comparative number for heat pumps is that temperature span is neglected. Carnot efficiency establishes the upper limit of COP based on span. Furthermore, it can be defined by span directly as

$$
C O P_{\text {max }}=\frac{T_{\text {cool }}}{T_{\text {hot }}-T_{\text {cool }}}
$$

for cooling, or

$$
\operatorname{COP}_{\max }=\frac{T_{\text {hot }}}{T_{\text {hot }}-T_{\text {cool }}}
$$

for heating.

where $T_{\text {cool }}$ is cold-side (or absorption) temperature and $T_{\text {hot }}$ is hot-side (or rejection) temperature.

The temperatures in either case are on an absolute scale, meaning that different applications cannot be compared to one another via COP without knowledge of span. For example, a household refrigerator generally rejects heat to an $80 \mathrm{~F}$ sink and absorbs heat at around $0 \mathrm{~F}$. $C O P_{\text {max }}$ for this is then about 5.75. In a room air conditioner heat is typically rejected at $100 \mathrm{~F}$ and absorbed at around $70 \mathrm{~F}$. $C O P_{\max }$ for this air conditioner is then over 17 . This suggests that defining heat pump efficiency as a percentage of Carnot efficiency is prudent as this term can be applied effectively to many various applications. 


\section{2 - Types of Heat Pumps}

The most common commercially used cycle is the vapor compression cycle, embodied in the figure 2 .

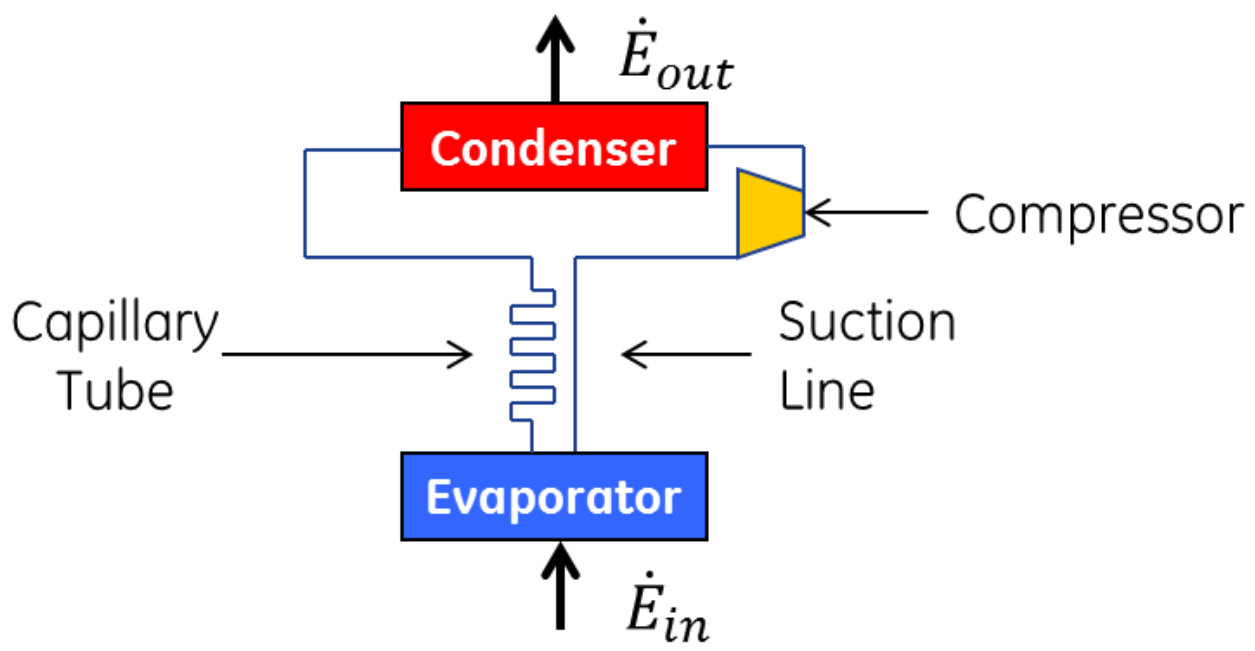

Figure 2: Vapor compression system

When a vapor is compressed adiabatically, temperature rises. This heat can then be rejected back to ambient and the compressed fluid can be decompressed. This causes the temperature to drop below ambient where it can be used to absorb heat from a load. A major issue with this technology is the working fluid that is used, which is generally hazardous to the environment, flammable, and tightly controlled. A version of the cycle has been proven capable of reaching $60 \%$ of Carnot efficiency (Pacific Northwest National Laboratory, 2010). In a household application the efficiency will be somewhat lower, due in large part to cost and scalability.

Other devices, such as Peltier coolers, can also create a temperature difference. This device uses a bimetal junction and an applied voltage to create a temperature difference, also called the thermoelectric effect. Thermoelectrics are not as commonly used as vapor compression, largely due to their low thermodynamic efficiency, which is only $10-15 \%$ of Carnot efficiency (Pacific Northwest National Laboratory, 2010).

Other more exotic heat pumps come in the form of thermoionic, thermotunneling, thermoelastic, and thermoacoustic technologies. Of these, thermoacoustics have proven the most successful, achieving around $20 \%$ of Carnot. The main interest in thermoacoustics is the theoretical efficiency limit, which approaches $100 \%$ of Carnot (Pacific Northwest National Laboratory, 2010).

Finally, another type of device uses the magnetocaloric effect to create a heat pump. A change in magnetic field creates a change in temperature which can be used to create a heat pump. This technology is expected to be capable of a $25-30 \%$ efficiency improvement over vapor compression when used in household refrigerators (Kuhn, 2010). This is equivalent to about 
$60 \%$ of Carnot efficiency (Pacific Northwest National Laboratory, 2010). When this figure is extended to include every household refrigerator, the energy savings are quite substantial. This technology is still in the prototype stage of development, but advances are being made quickly toward a commercially viable product.

\section{3 - Magnetocaloric Effect}

The magnetocaloric effect is the change in temperature of a material when it is exposed to or removed from a magnetic field. The effect is generally caused by the difference in entropy between a magnetically aligned and a magnetically random structure in magnetic materials. Pecharsky and Gschneidner (1999) created an excellent graphic depicting the transition.
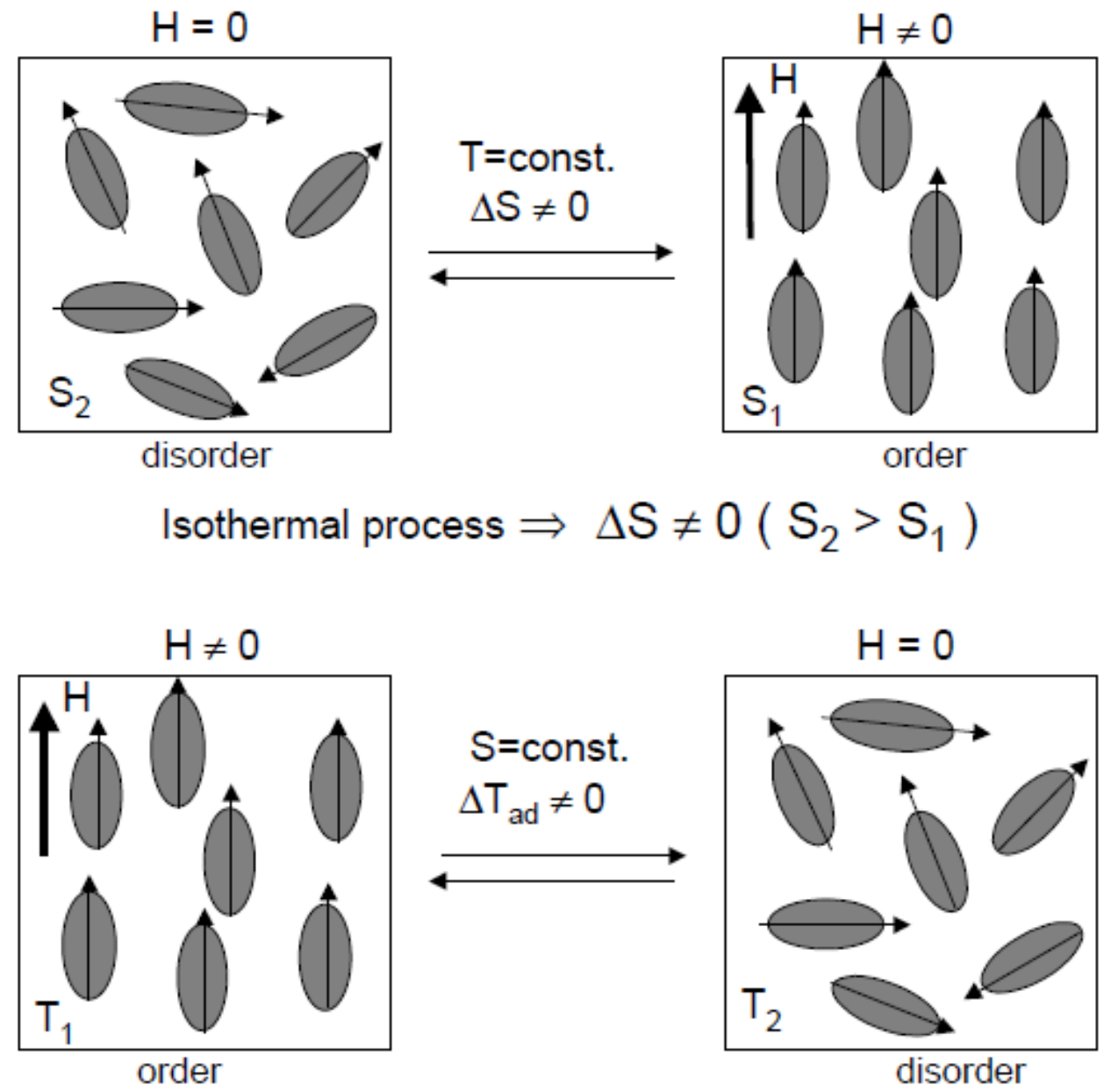

$$
\text { Adiabatic process } \Rightarrow \Delta \mathrm{T}_{\mathrm{ad}} \neq 0\left(\mathrm{~T}_{1}>\mathrm{T}_{2}\right)
$$

Figure 3: Magnetocaloric effect (Pecharsky and Gschneider, 1999, figure 1.1) 
Per figure 3, a magnetically aligned state has lower entropy level than a magnetically random state. If a material is forced into magnetic alignment with an external magnetic field in an adiabatic environment, the temperature will increase. Each particular magnetocaloric alloy is most active at what is known as Curie temperature. The magnetocaloric effect drops off as temperature deviates from this point. Materials are generally characterized by either adiabatic temperature rise or isothermal entropy change by magnetic field intensity and temperature. These relationships for Gadolinium are shown in figure 4.
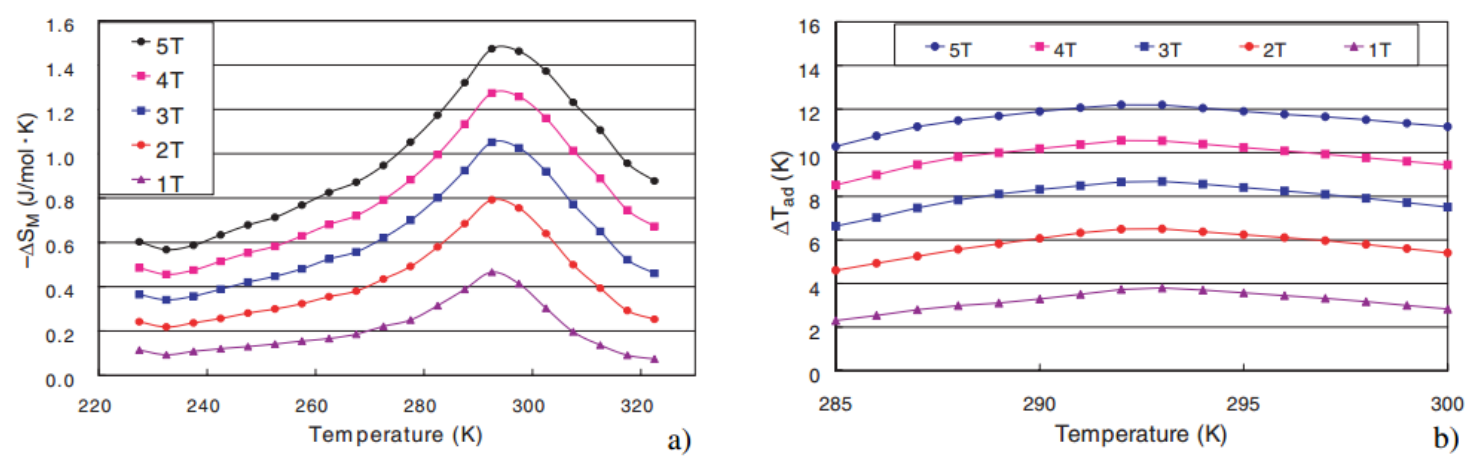

Figure 4: Isothermal entropy change (a) \& adiabatic temperature change (b) for Gd (Lee, 2004, figure 2)

Material curves vary by composition, but typically the maximum adiabatic temperature change does not exceed 3-4 $K$ at 1 Tesla. Because of the small temperature range of each material's effectiveness, specific materials must be developed which cover the entire desired span of any heat pump device. The different materials can be cascaded one after the other to create a larger span than any single material can achieve. An example of a possible five material cascade is shown in figure 5, of the composition $L a F e_{11.74-y} M n_{y} S i_{1.26} H_{1.53}$; where " $y$ " denotes changes in chemical make-up, shown in the subscripts of chemical formula. 


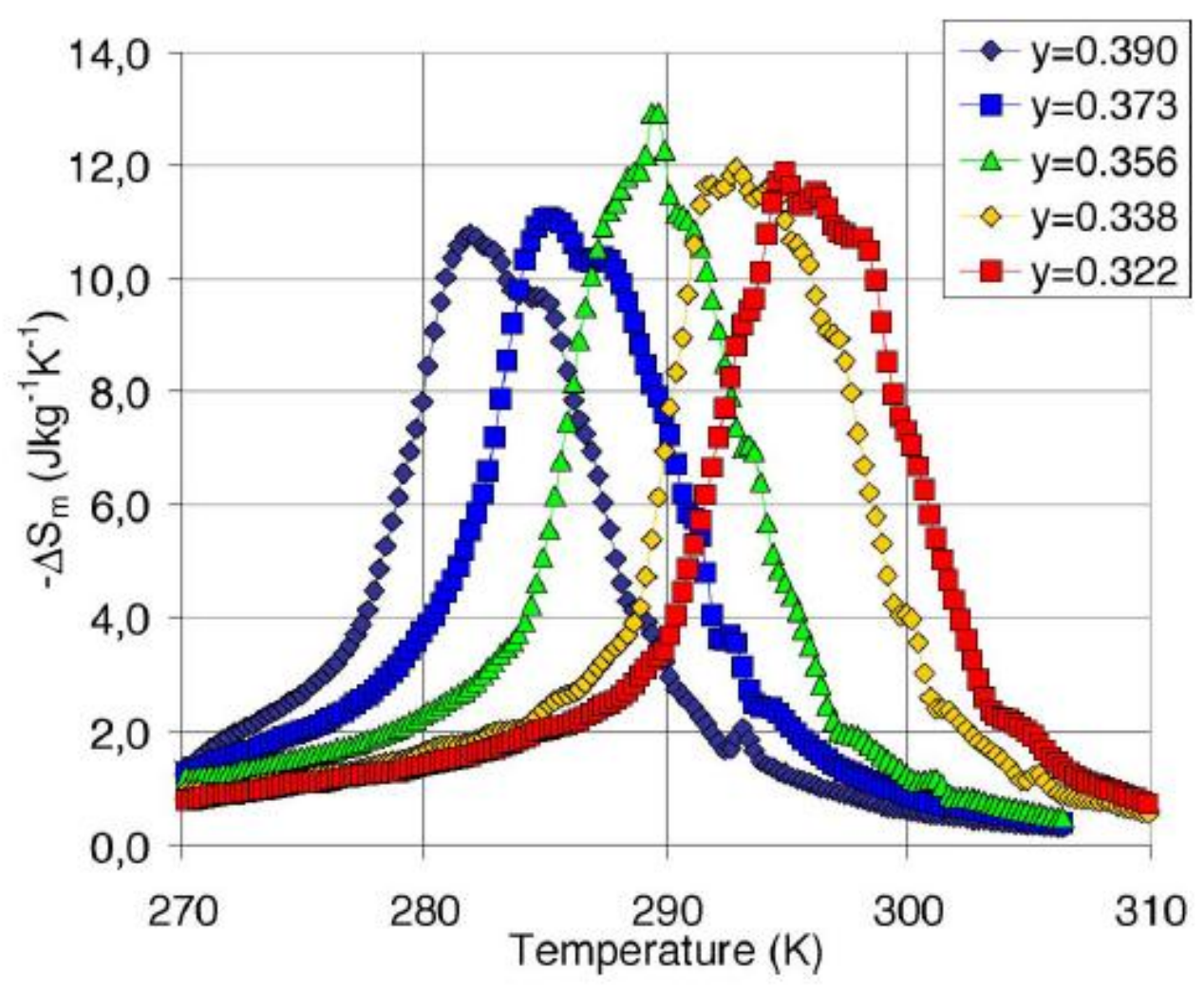

Figure 5: Isothermal entropy change vs. temperature at 1.6 Tesla (Barcza et al, 2011, figure 6)

Using this ladder of materials, each stage can operate near its Curie temperature. A temperature gradient will then span the regenerator during steady state operation. The number of stages required will be determined by the active span of each material, the total required span of the device, and the amount of overlap required between stages for stability.

\section{4 - Active Regenerator}

The regenerator is the heart of a magnetocaloric heat pump. It consists of an interface between solid magnetocaloric material and the working fluid. Because a packed bed can provide very high surface area per unit volume, it is a sensible choice for a regenerator to facilitate rapid heat transfer. Furthermore, irregular magnetocaloric material (MCM) particles are much simpler to create than spherical particles, so they are more widely used in prototypes. Pictures of two regenerators packed with MCM in clear containers are found in figure 6. 


\section{Spherical Material}
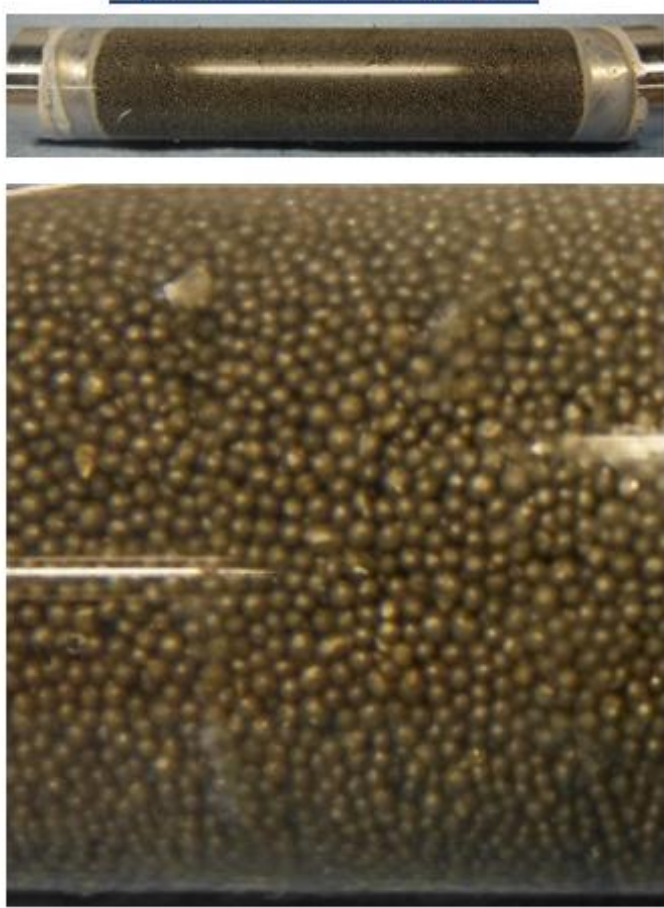

Pulverized Material
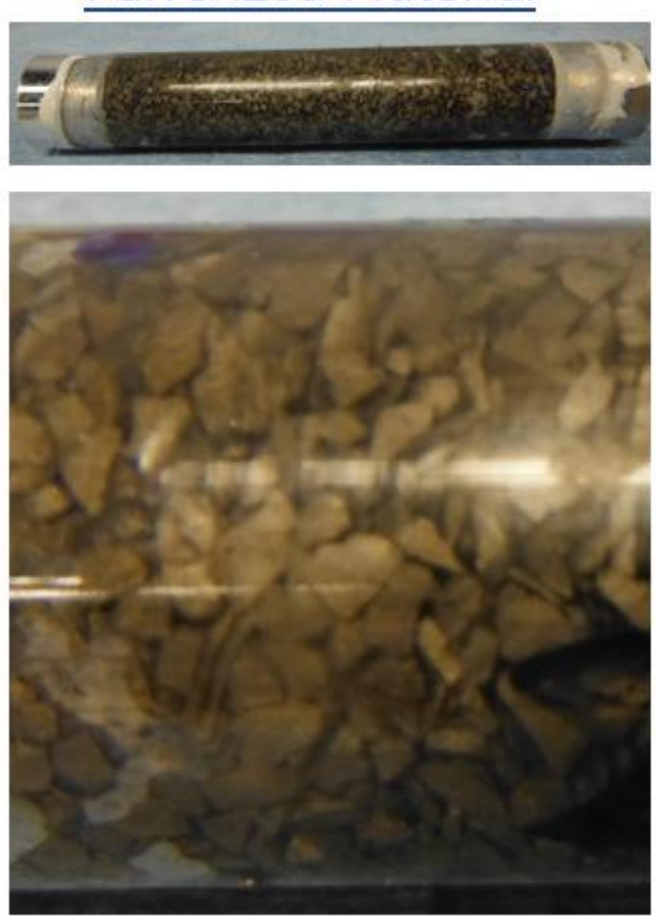

Figure 6: Photos of regenerators and particles

These regenerators are approximately four inches in length and are both packed beds of MCM particles. The particles in both cases are less than one $\mathrm{mm}$ in diameter. Although transparent, fluid occupies the spaces between particles in the photos. Two particle shapes are shown: from near-spherical (left) to crushed irregular material (right). In operation differences such as particle size and shape dramatically affect the operation of the machine. The particles on the right have a greater surface area and transfer heat at a much faster rate compared to the spherical particles on the left. Conversely, the spherical particle will have much less resistance to fluid motion.

This type of regenerator isn't to be confused with a passive regenerator as is found in a stirling heat engine, which simply stores heat cyclically. The terminology comes from the physical similarities of the two. Neglecting magnetic work input, they are both compact heat exchangers subjected to oscillating flow. The major difference is that and active regenerator both stores and generates heat cyclically. This makes an active regenerator a heat pump by definition, with magnetic work input, rejected heat output, and absorbed heat input. 


\section{5 - Magnetocaloric Heat Pump Cycle}

The embodiment of this magnetocaloric heat pump consists of a cold-side heat exchanger, a hot-side heat exchanger, an active regenerator, means for magnetizing and demagnetizing the regenerator, and means for oscillating the working fluid through the regenerator. Figure 7 shows one particular machine type operating at steady state. Flow is periodic, but an average temperature gradient exists as is shown; from cold (Blue) to hot (red).

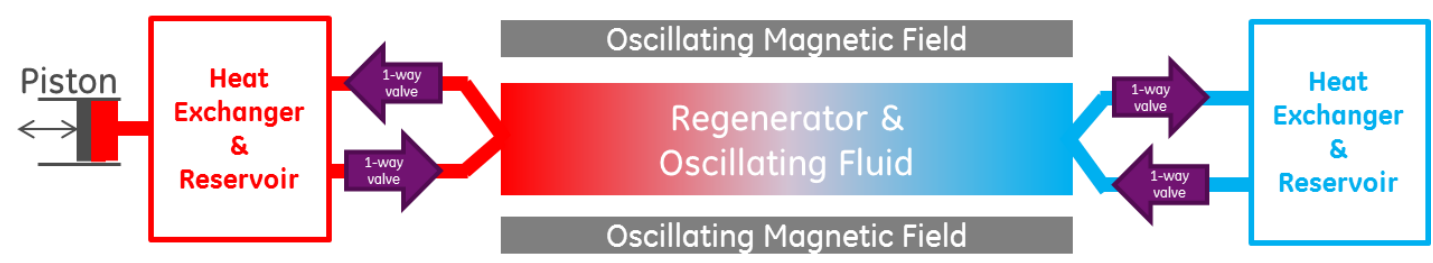

Figure 7: Magnetocaloric heat pump

One-way valves ensure that outgoing fluid must pass through a heat exchanger prior to returning the regenerator. Looking deeper, a typical cycle includes 4 segments.

1. Material is magnetized; MCM and working fluid temperatures rise to high level.

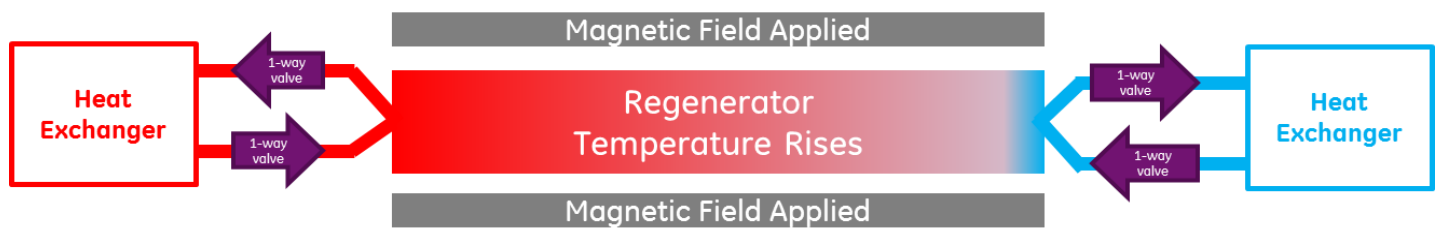

Figure 8: Magnetization segment

2. Some fluid is displaced. Ambient temperature fluid enters from the cold side, and heated fluid exits the hot-side. Additionally, the MCM is providing stored heat to the flowing fluid during this period.

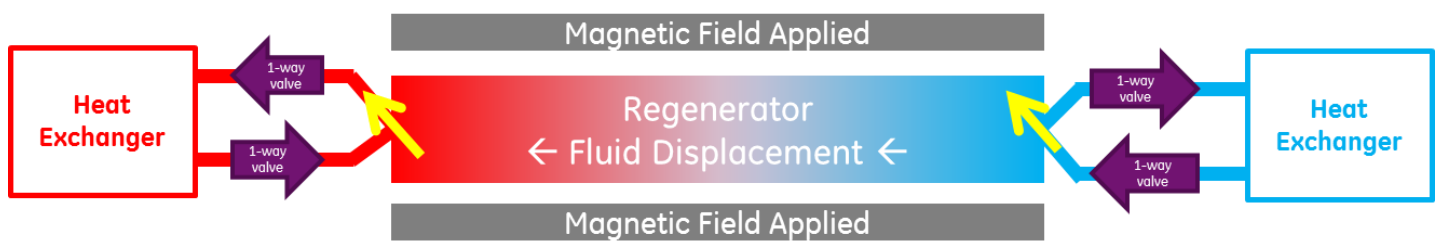

Figure 9: Warm flow segment

3. Material is demagnetized; MCM and working fluid temperatures fall to low level. 


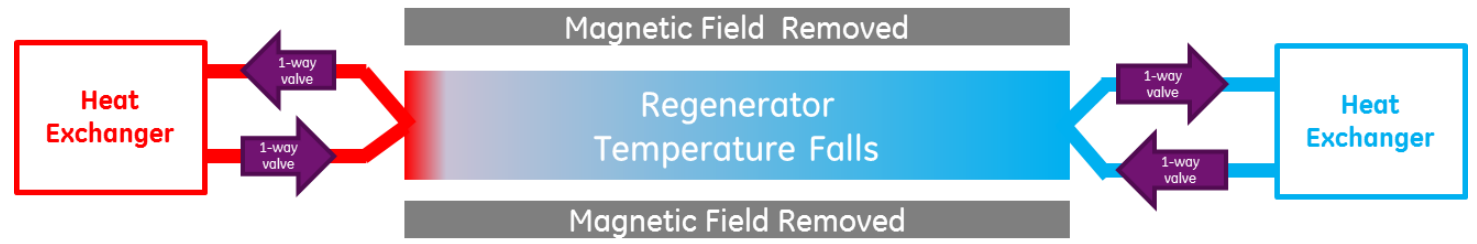

Figure 10: Demagnetization segment

4. Fluid is displaced in the opposite direction. Ambient temperature fluid enters from the hot side, and cooled fluid exits the cold side. Additionally, the MCM is receiving and storing heat from the flowing fluid during this period.

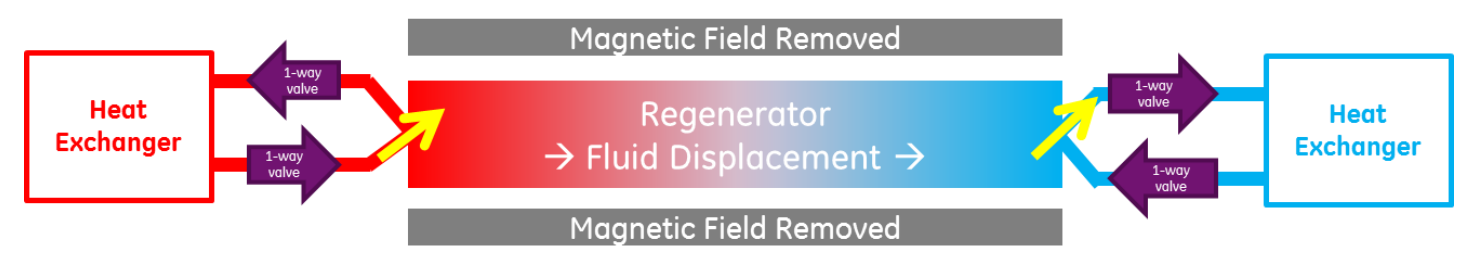

Figure 11: Cold flow segment

In summary, a cycle typically includes 4 segments:

1. Material is magnetized; material temperature rises to high level

2. Fluid passes through material, transferring heated fluid to hot-side. MCM loses heat.

3. Material is demagnetized; material temperature falls to low level

4. Fluid passes through material in opposite direction, transferring cooled fluid to cold-side. MCM receives heat.

In addition to these cycle segments, a dwell period can be inserted immediately after magnetization and demagnetization. This period of time can be used to allow the fluid and solid phases to exchange more thermal energy prior to the flow periods. 


\section{2 - PREVIOUS WORK RELATING TO HEAT TRANSFER DURING DWELL}

\section{1 - Experimental Work on Magnetocaloric Devices}

In order to design and optimize a magneto-caloric heat pump, the cycle must be modelled accurately. One method to achieve this is an experimental system optimization study. For instance, Gao et al. (2006) evaluated machine span and cooling load using different particle diameters and flow conditions. They determined performance trends as functions of these inputs, but did not go into any detail regarding the fundamental heat transfer relationships. Rowe and Tura (2006) operated another machine at different frequencies and cycle phase shifts to again determine trends in performance. As with many magnetocaloric experiments, the apparatus oscillated fluid with no dwell period. Similarly, Aprea et al. (2013) used a dimensionless approach to find performance trends. None of these experimental studies develop new insight into fundamental heat transfer.

Another method to optimize the system is to mathematically model each segment of the cycle. Petersen et al. (2008) built a two dimensional numerical model to optimize a fin-type regenerator system. This study placed little emphasis on heat transfer. A similar study was performed by Nielsen et al. (2009), with the main difference being the addition of heat leak. Models have also been created for packed bed-type regenerators. One such example is that of Bouchard et al. (2009), who built a CFD model using spherical geometry. They used the model in order to predict high level performance. The main concern for model quality in this case was matching MCM temperature response curves; no quantitative heat transfer studies were performed. A more general approach to the problem allows for irregular particles to be modeled instead of perfect shapes.

To take this a step farther, the transient element of time can potentially be simplified. Kays and London (1984) found heat transfer correlations with spherical particles and other porous media and extended their findings to include frequency-based average heat transfer. Within this approach "dwell time" is described as total occupation time of fluid within the material in a regenerative cycle. This definition differs from that used in this work which describes "dwell time" as time with no bulk fluid motion.

Within any of the time stepping models, several main components are needed to accurately portray a full cycle. Firstly, material temperature response curves are needed, which are generally available for magneto-caloric materials. Secondly, heat transfer correlations for flowing fluid within a packed particle bed are needed, which are again available due to previous work on similar steady state processes. Finally, heat transfer correlations for no bulk fluid 
movement are needed in a six segment cycle. This component is lacking and is the subject of this work.

\section{2 - Experimental Studies on Forced Convection}

Previous work on forced convection within packed beds can offer some insight to the heat transfer occurring during a dwell period. In some cases, the results from flowing fluid experimentation can be extended to a Reynolds number of zero. This depends entirely on the range of Reynolds numbers that have been studied.

For example, Denton et al. (1963) studied the transfer between a packed sphere bed and flowing fluid. They came up with the following relationship:

$$
\text { St } \operatorname{Pr}^{\frac{2}{3}}=0.23 \operatorname{Re}^{-0.3}
$$

where St is Stanton number, Pr is Prandtl number, and $R e$ is Reynolds number.

This works well for the flow components of the cycle given that the Reynolds number is high. The issue is that Stanton number and therefore heat transfer coefficient reduce to zero as Reynolds number and flow velocity reduces to zero. Clearly this is not accurate for stagnant fluid, as a temperature difference will always drive some form of heat transfer. In this case a limit value must be added to account for stagnant fluid conduction or convection. Additionally, this correlation is valid only for spherical particles.

Whitaker (1972) also performed a forced convection study on randomly packed spheres, finding the following relationship:

$$
N u=2+\left(0.4 \operatorname{Re}^{1 / 2}+0.06 \operatorname{Re}^{\frac{2}{3}}\right) \operatorname{Pr}^{0.4}
$$

This study included experimentation down to a Reynolds number of 3.5, and includes a nonzero solution to the zero velocity case. At zero velocity Nusselt number is limited to a minimum value of two. The issue is that this constant was not fitted to experimental data for a static fluid case, but instead added to enforce known realities. By definition, Nusselt number is

$$
N u=\frac{h D}{k} .
$$

where $\mathrm{h}$ is convection coefficient, $\mathrm{D}$ is particle diameter, and $\mathrm{k}$ is fluid thermal conductivity.

Solving for convection coefficient with $\mathrm{Nu}=2$ yields 


$$
h=\frac{2 k}{D}
$$

Looking at this as a pure conduction problem, the fluid conduction length is equal to D / 2 . Considering the small size of interstitial voids in a packed bed, this is a very conservative value. This constant value is seen again in a correlation by Kunii and Lievenspiel (1969),

$$
N u=2+1.8 \operatorname{Re}^{1 / 2} \operatorname{Pr}^{1 / 2},
$$

and again in a widely used correlation by Wakao et al (1979),

$$
N u=2+1.1 \operatorname{Re}^{0.6} \operatorname{Pr}^{1 / 3}
$$

Figure 12 depicts the experimental confidence ranges in an experiment by Wakao and Kagei (1982)

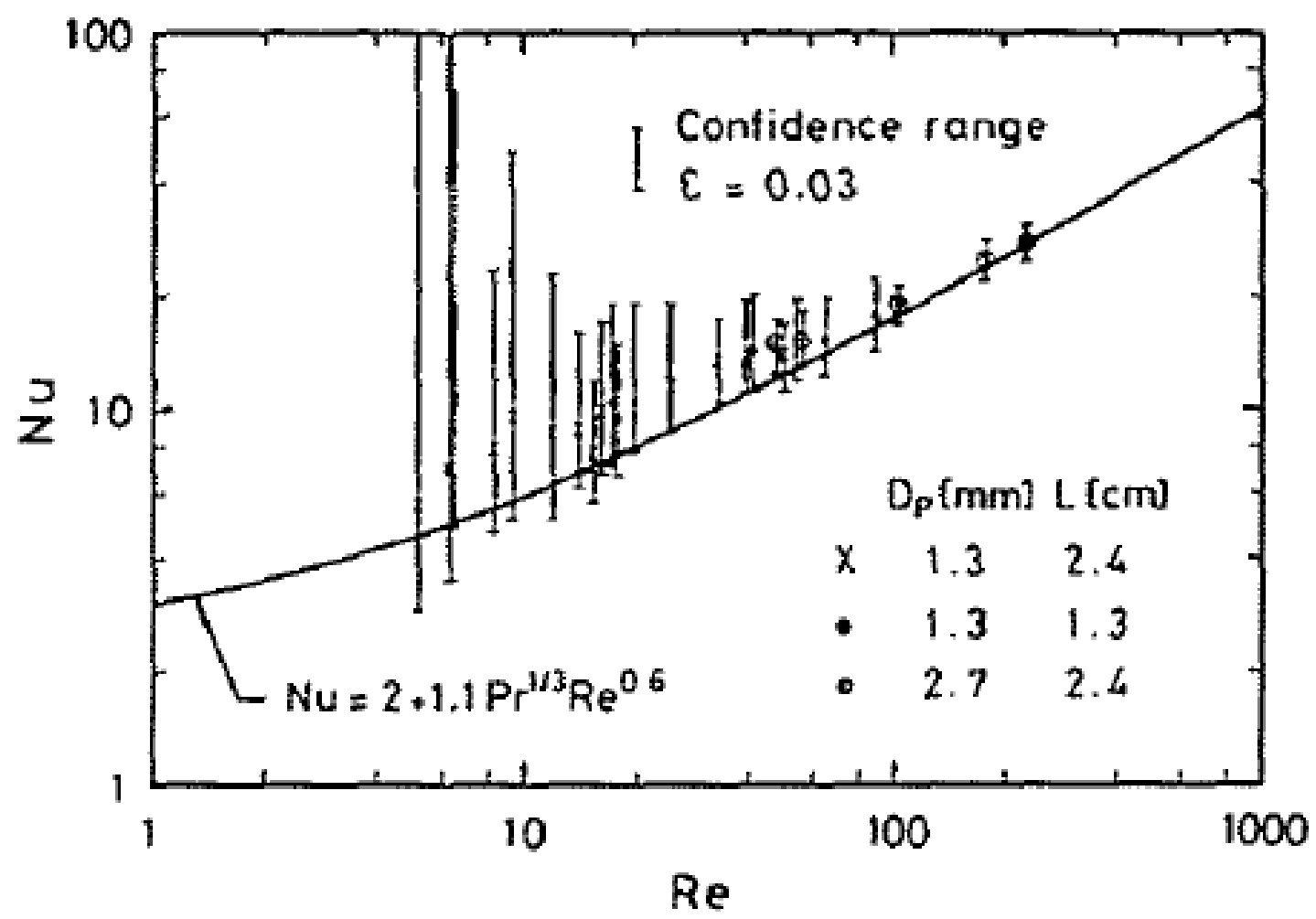

Figure 12: Experimental confidence ranges (Wakoa and Kagei, 1982, figure 3)

Notice the large confidence bounds as Reynolds approached zero. Additionally, following the centers of the confidence bounds toward $\mathrm{Re}=0$ suggests that the Nusselt number constant should have a value of about 10 . Some correlations do exist with larger constants than two. An 
analytical solution proposed by Gnielinski (1978) is one such example. When Reynolds number is set to zero, the correlation reduces to

$$
N u=2(1+1.5(1-\varepsilon))
$$

Setting void fraction to a value of 0.42 for a typical random particle pack yields $\mathrm{Nu}=3.8$. This comes from analytical work on spherical particles. The corresponding conduction length for this Nusselt number is D / 3.8. In close agreement with this result is a correlation by Gunn (1978). With a Reynolds number of zero, the Gunn correlation reduces to

$$
N u=7-10 \varepsilon+5 \varepsilon^{2} .
$$

Setting void fraction again to a value of 0.42 yields $\mathrm{Nu}=3.7$. This comes from extensive testing, and has been stated to be accurate down $R e=0.1$. The main issue with these studies is the experiment itself which depends on fluid flow to measure heat transfer rate. These provide a general prediction for heat transfer during a dwell period, but no true dwell experiment.

\section{3 - Experimental Studies on Free Convection}

Another heat transfer case with similarities to the dwell period heat transfer is free convection. As Rayleigh number approaches zero, a similar conduction limit will be reached. The interstitial voids between particles can be approximated as cavities, for which prior work has been done. Globe and Dropkin (1959) performed experiments on cavities heated from below, and created the following correlation:

$$
N u=0.069 \operatorname{Ra}^{\frac{1}{3}} \operatorname{Pr}^{0.074}
$$

As seen before in some forced convection studies, this function approaches zero as Rayleigh number approaches zero. This correlation is valid only when strong convection currents exist in steady state, when Rayleigh number is large. Since very small closely packed particles are the subject of the current work, Rayleigh number will be extremely low. However, as discussed in the context of forced convection, heat transfer will not cease for this low Rayleigh number case. This and other free convection cases share another issue when compared with the current work, and that is the method of heating. Many studies involve steady state heating from a single direction. In the current work, transient heating occurs from all directions.

Another analogue to the current work is natural convection on a spherical particle. Churchill (1977) developed a correlation for this case, 


$$
N u=2+\frac{.589 R a^{\frac{1}{4}}}{\left[1+\left(\frac{.469}{\operatorname{Pr}}\right)^{\frac{9}{16}}\right]^{\frac{4}{9}}} .
$$

The constant value of two again shows up, and the reasoning behind the constant is also quite similar. Regardless of convective currents, heat will conduct out of the sphere with any temperature difference. Yet as discussed earlier, the value is very conservative.

A somewhat similar convection case comes about in the food processing industry, in heating food pieces. Awuah et al. (1993) provide one such example using carrots and potatoes. Their experiment consisted of food items (carrots \& potatoes) contained in a cylinder in a bath. Nonforced heat transfer occurred within the cylinder, but the fluid phase was by no means stagnant. These types of experiments differ fundamentally in size scale, time scale, and void fraction. Due to the nature of the process, natural convection was the overwhelming means for heat transfer.

Major differences are present between all of these cases of natural convection and the heat transfer occurring during a dwell period. Most predict a steady state heat transfer coefficient with a constant driving temperature difference from a single direction. Transient temperature differences, irregular voids, and heat from all directions make the heat transfer during a dwell period unique.

\section{4 - Objectives}

The main objective of this work is to analytically describe and to experimentally measure the transient heat transfer occurring during a dwell period between the MCM and the working fluid. The MCM is embodied as a packed irregular particle bed.

First, the usefulness of a dwell period must be proved. This will be done by simulating a basic cycle qualitatively, both with and without a dwell period. Next, the dominant mode of heat transfer must be determined during the dwell period, as either conduction or convection. This will facilitate the creation of an analytical model, which can be used to determine the time and temperature scales of interest. In addition, an existing magnetocaloric test fixture must be characterized to assess physical measurement capabilities. The existing fixture will be modified as needed to measure interstitial fluid temperature within the regenerator over time during a dwell period after magnetization. This test will be performed across a design space of different particle diameters, magnetocaloric materials, and working fluids. An additional pressure drop test will be used to independently estimate the surface area, or irregularity, of each set of particles.

Using the results from the experiment, the temperature profile of the fluid over time can be used to determine heat transfer rate for each case. This heat transfer rate will be compared 
with the predictions of the analytical model, and the model will be modified if necessary. If possible a single correlation will be determined to model heat transfer rate for both stagnant and flowing conditions. 


\section{3 - THEORY AND MODELING}

\section{1 - Demonstrating the Usefulness of a Dwell Period}

A large assumption often made with the typical cycle is that heat is transferred nearinstantaneously from MCM to fluid, and that both fluid and solid can be considered equal in temperature. This approximation cannot be made for all cases and it can skew results significantly. In fact, regenerators with slower heat transfer rates may benefit by waiting for some period of time before displacing fluid. This time allows the fluid and solid to approach thermal equilibrium.

\subsection{1 - Basic Two-Mass Time Stepping Model Setup}

To demonstrate, a simple thermal model was created for a half cycle using basic square-wave input profiles; shown in figure 13.

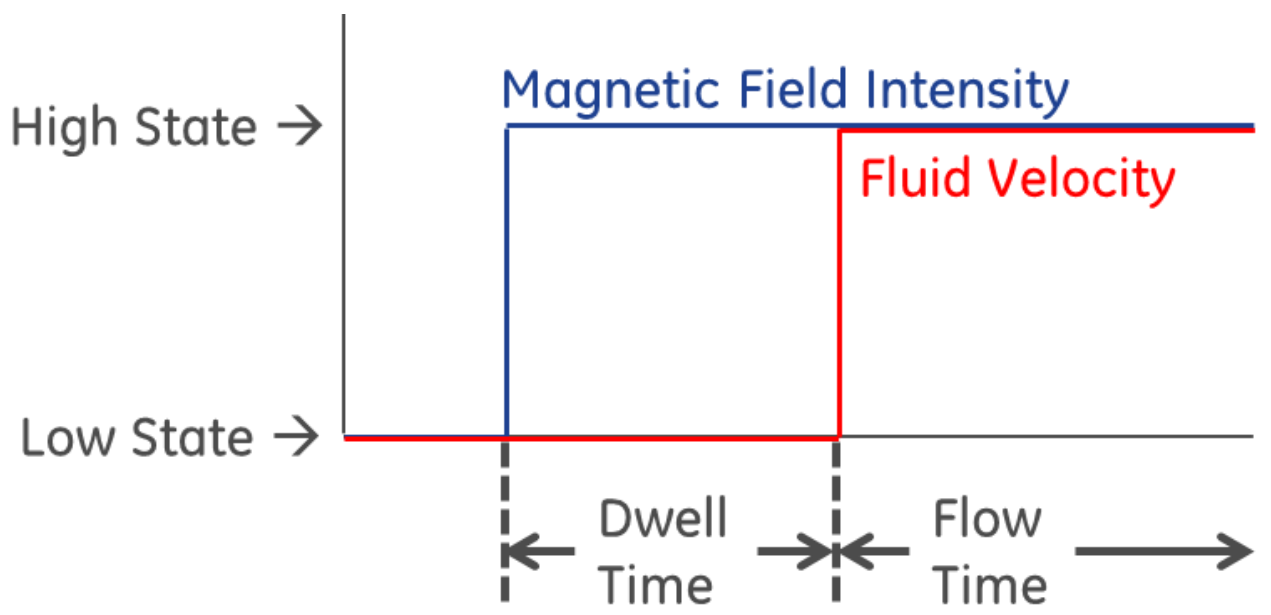

Figure 13: Half cycle state diagram using square-wave input

The step-change in magnetic field was set to produce an arbitrary $3 \mathrm{~K} \mathrm{MCM}$ (magnetocaloric material) change in temperature from $300 \mathrm{~K}$. This comes from typical material response to a magnetic field. The regenerator was split into 10 segments axially, and time stepping was set such that a full segment's fluid volume is displaced during each step forward in time. The length of each segment was set to $0.010 \mathrm{~m}$, and the time step length was set to $0.01 \mathrm{~s}$. This makes the simulation extremely simple to set up and run for qualitative results. Each axial position is occupied by both a fluid and solid node mass, as is shown in figure 14. 


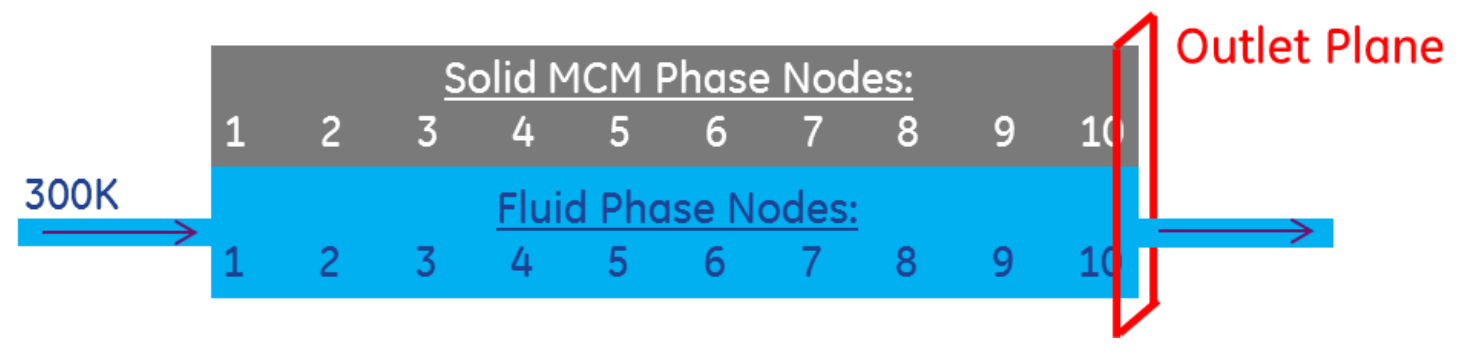

Figure 14: Demonstrative model layout

Void fraction was set to 0.37 for a close random pack of spheres. MCM density and thermal heat capacity were set to $7900 \frac{\mathrm{kg}}{\mathrm{m}^{3}}$ and $1000 \frac{\mathrm{J}}{\mathrm{kgK}}$ respectively. Working fluid density and thermal heat capacity were set to $981 \frac{\mathrm{kg}}{\mathrm{m}^{3}}$ and $4200 \frac{\mathrm{J}}{\mathrm{kgK}}$ respectively. Heat transfer coefficient multiplied by area, or UA, between fluid and solid for each segment was set arbitrarily at $100 \frac{\mathrm{W}}{\mathrm{K}}$ and $50 \frac{\mathrm{W}}{\mathrm{K}}$ for flow and dwell conditions respectively. Temperature change between time steps was then determined according to fluid flow condition. For no fluid flow, the following applies for each segment.

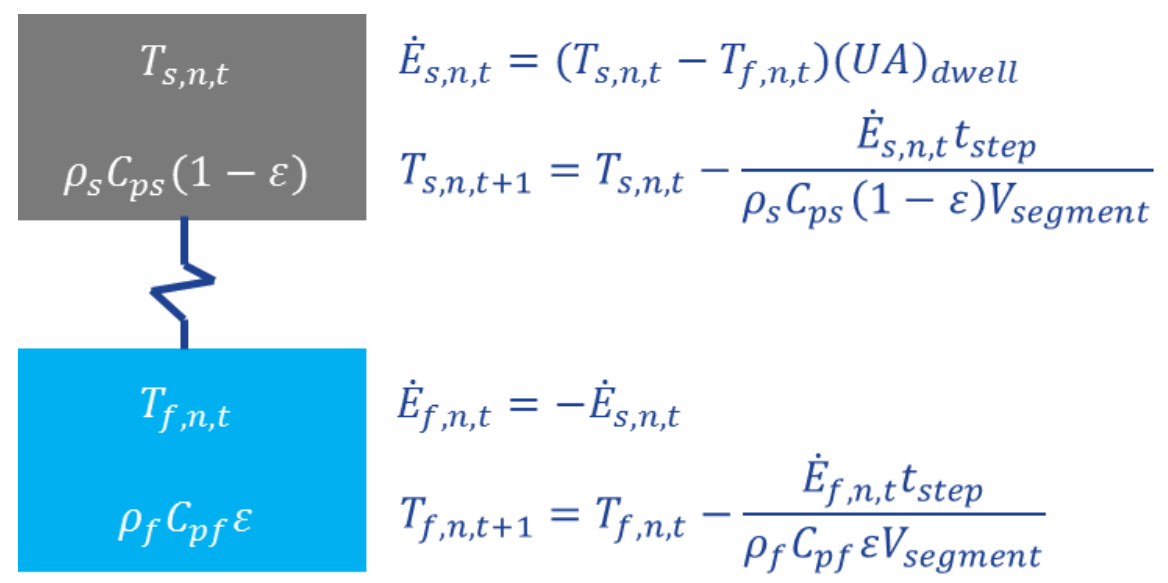

Figure 15: Rate of heat transfer during dwell period

where $\dot{E}$ is heat transfer rate into the subscripted node at the subscripted relative axial position and time step, $T$ is temperature of the subscripted node at the subscripted relative axial position and time step, $(U A)_{d w e l l}$ is heat transfer coefficient multiplied by heat transfer area during dwell, $t_{\text {step }}$ is time step size in seconds, $C_{p}$ is heat capacity, $\rho$ is density, $\varepsilon$ is void fraction, $V_{\text {segment }}$ is volume of regenerator segment, $s$ or $f$ as subscripts indicate solid or fluid phases respectively, $n$ or $n+1$ as subscripts indicate relative axial position to current location, and $t$ or $t+1$ as subscripts indicate relative time step position to current time step.

Each segment has an established thermal mass which remains constant. The temperature difference between the fluid and solid within the same axial location drives heat transfer. This heat transfer rate determines the temperatures in the following time step, $t+1$. Axial 
conduction and heat leak are neglected to maintain model simplicity. The condition of fluid flow is similarly defined, as follows.

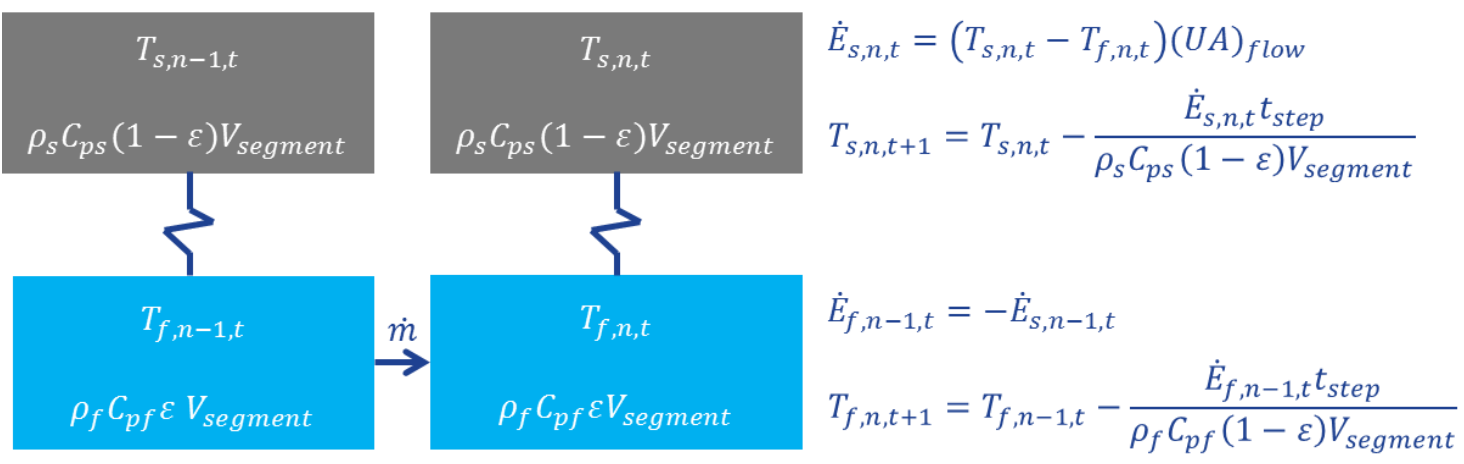

Figure 16: Rate of heat transfer during flow period

In this case heat transfer rate is still defined by the same temperature difference, but the resulting fluid temperature is shifted to the right by one node. Again, this creates an extremely basic heat transfer model to be used qualitatively. This makes the time and temperature scales arbitrary.

\subsection{2 - Two-Mass Time Stepping Model Output}

If the dwell period is set to zero, the outlet fluid temperature has the form shown in figure 17.

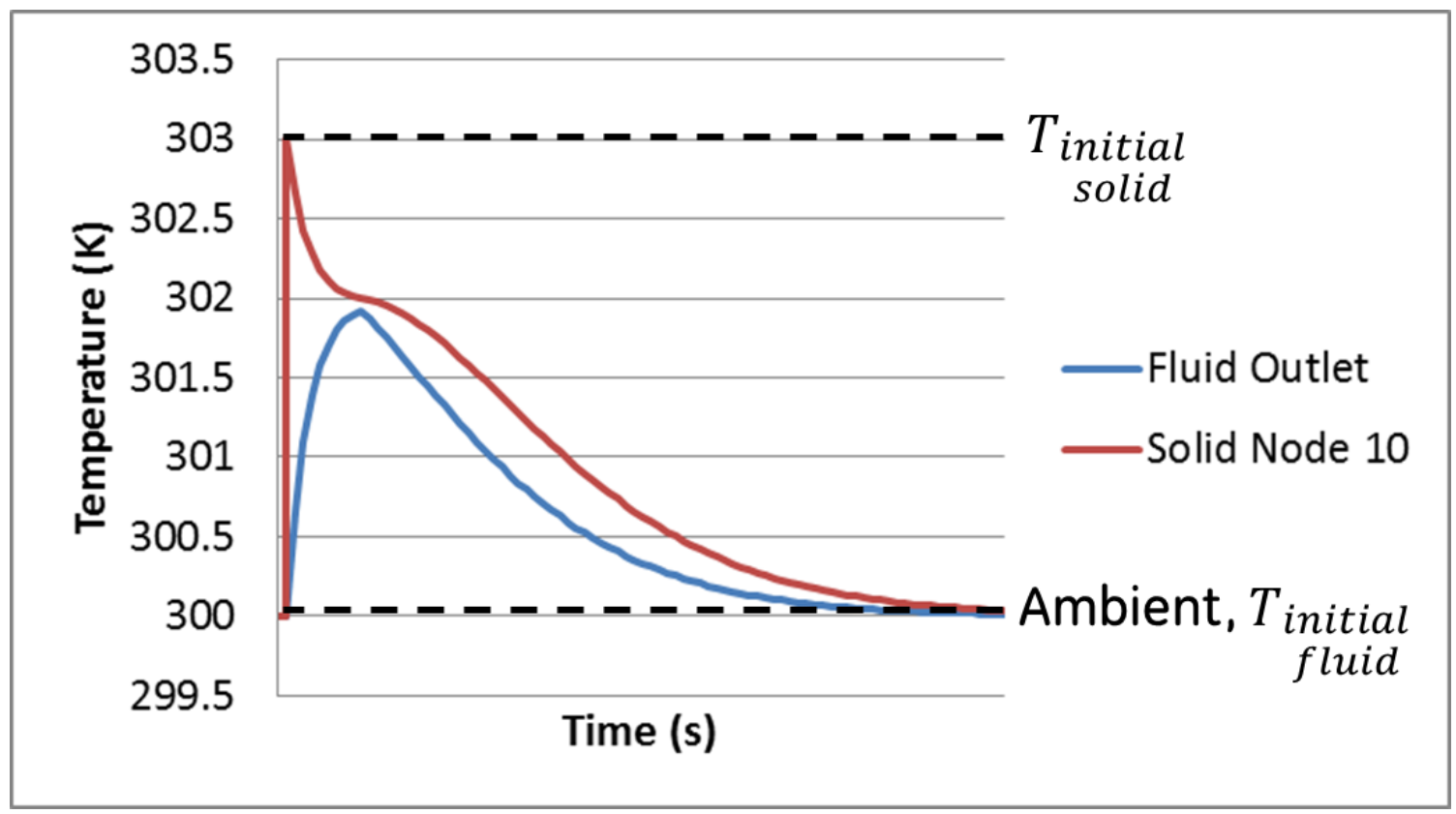

Figure 17: Outlet temperature profile with no dwell period

The first fluid to exit has not had any time to receive heat from the magnetocaloric material; therefore the starting fluid temperature is ambient. By not flowing fluid immediately after the 
solid material temperature change, the average outlet temperature can be increased. During dwell, the fluid will approach the thermal equilibrium temperature defined by the thermal masses of the solid and fluid materials. If an extended dwell period were used, achieving equilibrium prior to flow, the resulting temperature profile at the regenerator exit would look like that shown in figure 18.

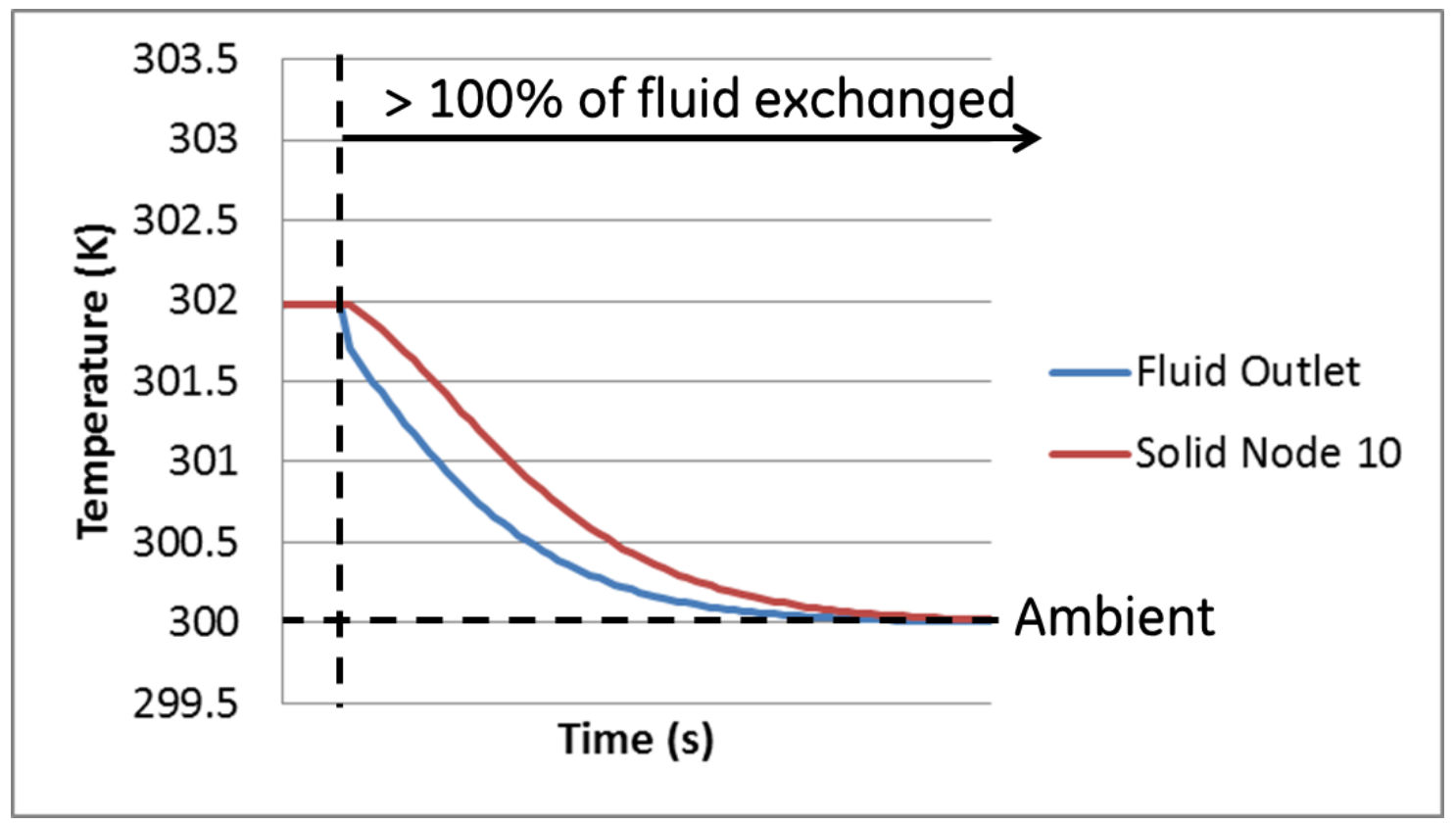

Figure 18: Outlet temperature profile with a dwell

The fluid volume that started out in the regenerator exits the regenerator at constant temperature, corresponding to the equilibrium temperature. After this fluid is flushed out, the outgoing fluid temperature begins dropping towards the ambient inlet temperature. A comparison of the two cases is shown in figure 19. 


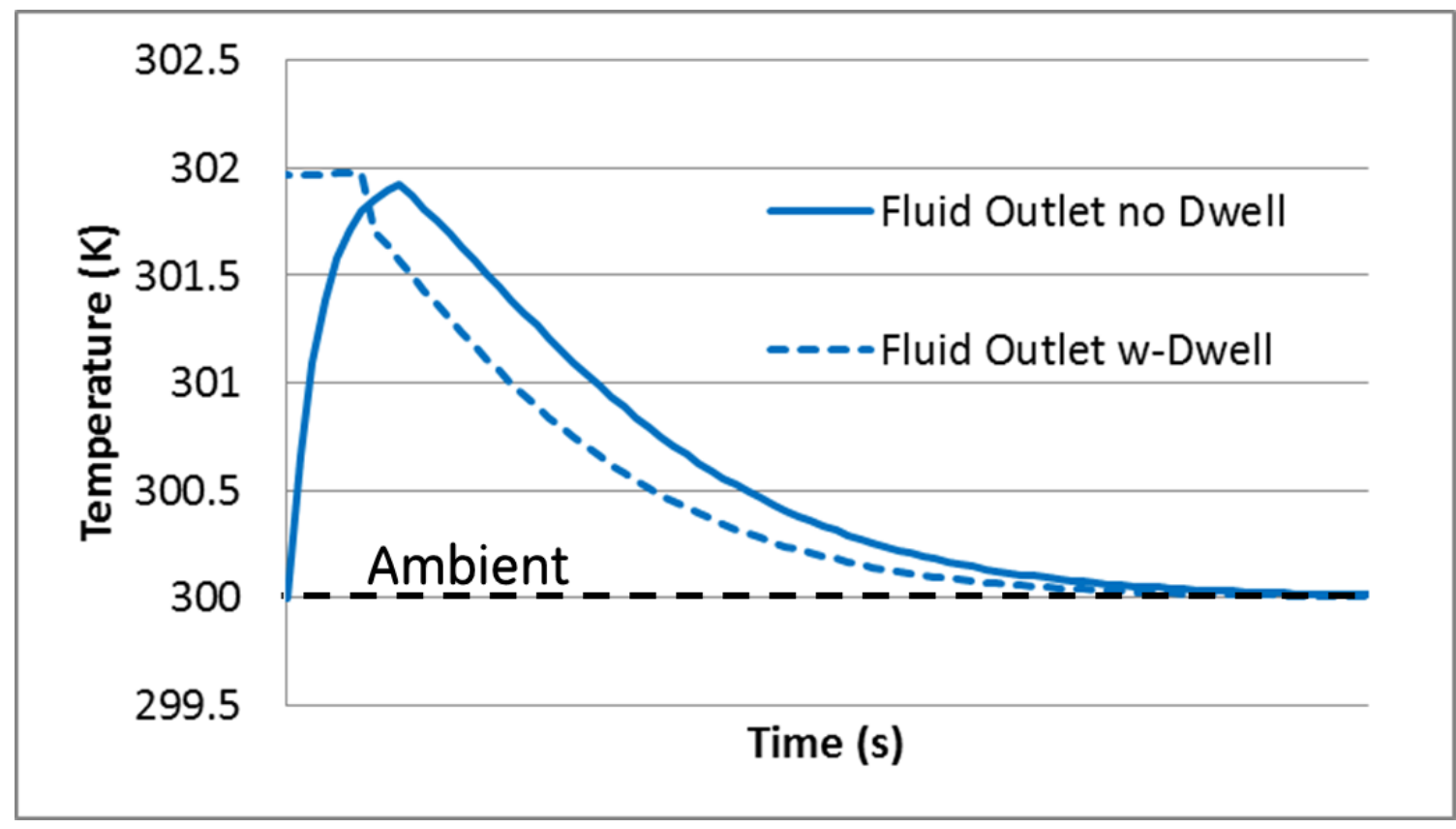

Figure 19: Outlet temperature profile comparison

The area under the dwell-inclusive temperature curve is initially higher than that of the cycle without a dwell period. This area below the curve corresponds to energy contained in the purged fluid. This integral will be equal for the two cases if taken out to equilibrium. Using a small amount of dwell prior to flow-through allows for higher average temperature of the exiting fluid, given that flow stops before ambient is reached. This can lead to an increase in thermal load per cycle for the same physical device. Likewise, more energy can be removed from each stage in the latter and moved. This can also lead to a decrease in the required number of stages to achieve a total temperature span. A reduction in stages and materials inherently leads to a more economical and manufacturable product.

With this in mind, a revised cycle could include six segments:

1. Material is magnetized; material temperature rises to high level

2. Dwell; fluid temperature rises

3. Fluid passes through material, transferring heated fluid to hot-side

4. Material is demagnetized; material temperature falls to low level

5. Dwell; fluid temperature falls

6. Fluid passes through material in opposite direction, transferring cooled fluid to cold-side

Another benefit of this type of cycle is that regenerator geometries with slower heat transfer rates can be used. These geometries tend to have less pressure drop, which is a very important component of overall machine efficiency. Even if a dwell period is not intentionally added there will be times in most cycles when there is no bulk fluid motion. An accurate model for heat transfer during this time is necessary for an accurate total system model. 
Now that the potential usefulness of a dwell period has been demonstrated, an analytical model can be made to calculate heat transfer coefficient. In order to make such a model, the dominant mode of heat transfer needs to first be determined.

\section{2 - CFD Study to Determine Dominant Heat Transfer Mechanism}

Is the heat transfer in the current case truly conduction limited, or are buoyant convection currents being forming within the interstitial voids? The driving force for creating a convective current is a temperature difference, and some amount of time is required for this force to accelerate the fluid to form a current. In this case the time scale is extremely rapid due to the short conduction lengths. The packed beds being used have particle diameters less than one $\mathrm{mm}$. In addition, the transient nature of the case means that the driving temperature difference decays with time. Finally, viscous resistance counters the buoyant force and any fluid motion; meaning that smaller cavities require much stronger buoyant forces to create convective currents than larger cavities. These facts qualitatively support the assumption of pure conduction.

\subsection{1 - Setup \& Assumptions}

In order to quantitatively address the question of convection versus pure conduction a particlescale model was built for use in a CFD (Computational fluid dynamics) study. Particles were approximated as spherical and positioned in a hexagonal close pack (HCP). The geometry of one particular case is presented in figure 20.

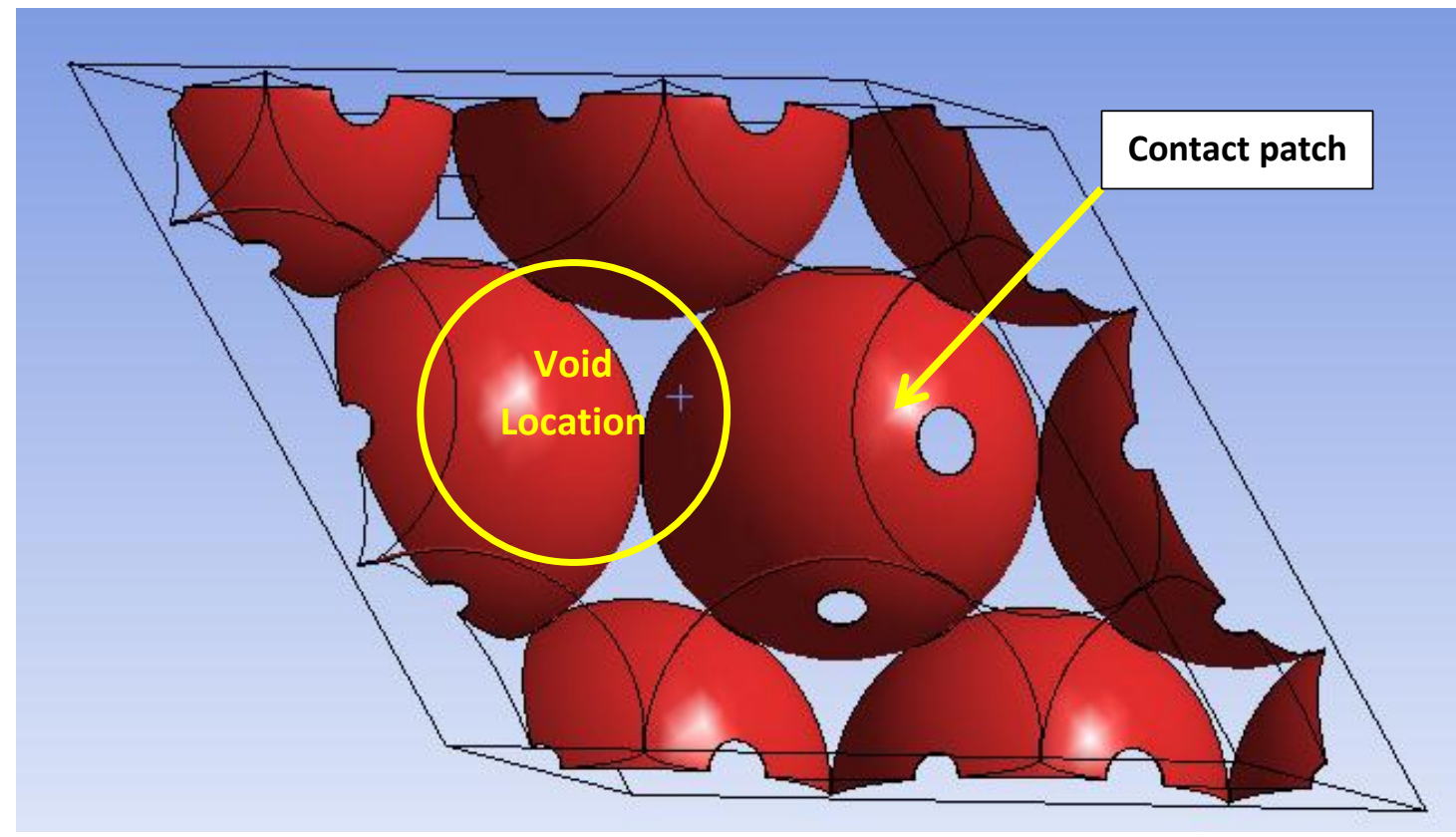

Figure 20: CFD Geometry 
A single particle location was left void to approximate the largest likely void in a random pack situation. This should create the scenario most likely to create free convection currents using the given particle diameter. This raises the overall simulated void fraction from 0.26 to 0.36 , which is approximately the lower limit for a close-random-pack of spheres (Scott and Kilgour, 1969).

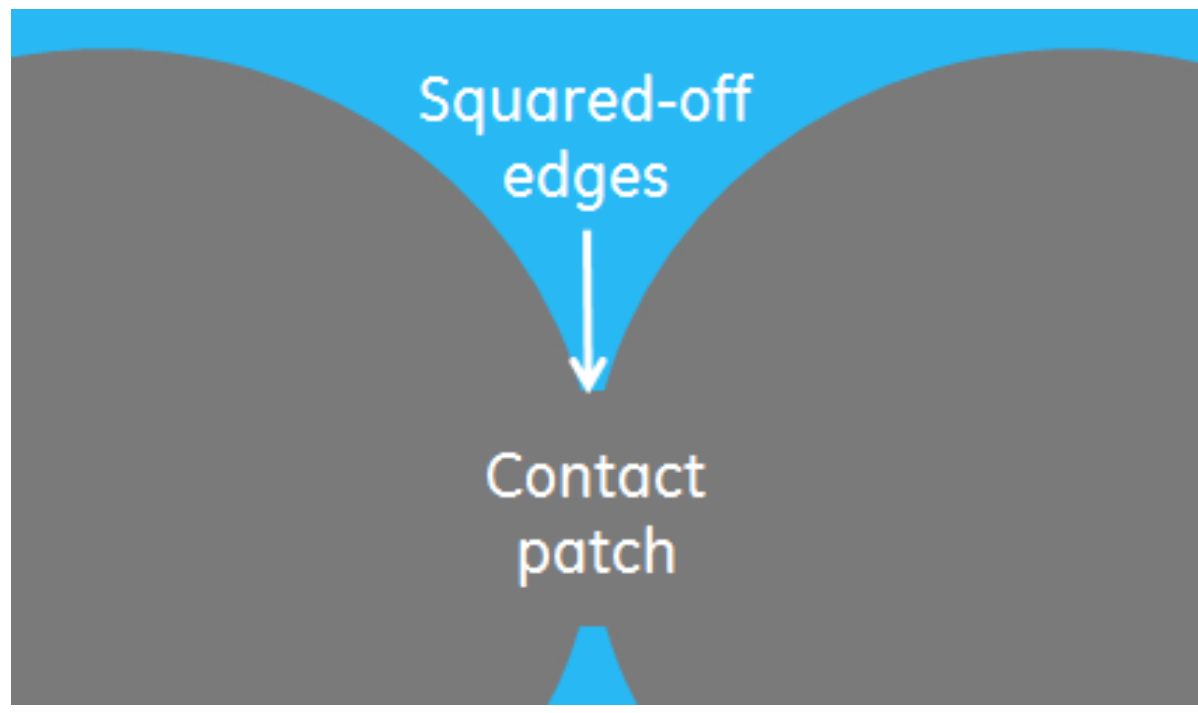

Figure 21: Contact patch treatment

The contact patches between touching particles were approximated as circular, with a squaredoff flat surface. This was done in order to improve simulation speed and mesh quality. The liquid trapped in the zone between particle contact patches does play an important role in interparticle conduction, but much less in particle to fluid heat exchange. The fluid in this zone changes temperature almost instantaneously due to a large surface area to mass ratio. The assumption is that it does not significantly alter the bulk temperature rise rate in the larger voids.

The model was set up with symmetry on the four vertical planes, and two walls on the top and bottom. This allows convection currents to form on the vertical boundaries, where it is expected. Symmetry planes mirror flow on the interface, while walls act as a physical obstruction. These boundary conditions were put in place with the assumption of pressuremirroring, or having the same pressure on all faces. Pressure mirroring will occur in an infinitely large packed bed of this condition. Pressure mirroring will not hold true near walls or when the time scale allows large-scale convection through the packed bed from void to void. As the intent of this simulation was to determine void-scale convection, this is an acceptable assumption.

Gravity was set perpendicular to the viewing plane (Front of figure 20). The intent was to monitor average bulk fluid velocity and temperature over time, and to visually determine the prevalence of convection currents. If currents and mixing are negligible a pure conduction 
assumption can be made for the fluid. Otherwise, the shape of convection currents may help in determining the best model to utilize as a starting point in creating an analytical model.

Water is set as the interstitial fluid using constant properties with the exception of density. Density must be modeled as a function of temperature for free convection to occur. Viscosity, heat capacity $\left(C_{p}\right)$, and thermal conductivity were approximated as constant due to the small temperature changes involved.

A $3.5 \mathrm{~K}$ step-change in temperature was used to approximate the magneto-caloric effect. This was used assuming that the time scale of magnetization is much smaller than that of heat transfer. Since a step-change is an unnatural phenomenon, it will lead to incredibly large heat flux values at the solid-fluid interface. This will likely create inaccuracies over the first few time steps, and for this reason the first time steps should not be considered.

In operation, the absolute temperature is likely to fluctuate with load and sink temperature variation. Experimentally determined temperature vs. thermal heat capacity for high and low magnetic field states for one particular magnetocaloric material is plotted in figure 22.

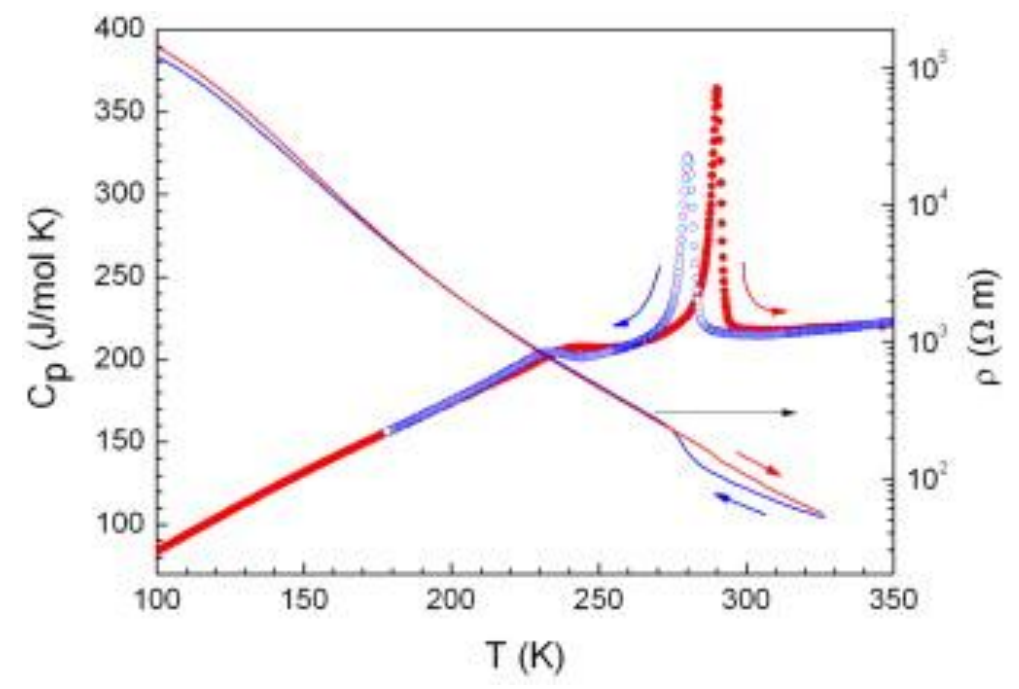

Figure 22: Cp vs. temperature curves for high and low magnetic states (Aliev et al., 2011)

The materials that are used have a highly variable thermal heat capacity, which peak near Curie temperature. With this in mind, the simulation was run using tabulated $C_{p}$ data near the peak region, and also $1 \mathrm{~K}$ off-peak. The curve is much flatter at this off-peak point. Each of these was run in both heating and cooling, corresponding to the low and high field states in the cycle. This yields four runs per case:

1. Heating, Peak $C_{p}$

2. Heating, Flat $C_{p}$

3. Cooling, Peak $C_{p}$

4. Cooling, Flat $C_{p}$ 


\subsection{2 - Model independence}

Several studies to ensure model independence were run. The monitored output for determination of independence was time to an average fluid temperature change of $1 \mathrm{~K}$. Each case was run four times, corresponding to the $4 \times 4$ matrix of heating/cooling and absolute starting temperature. The first check was for time step size independence, seen in figure 23.

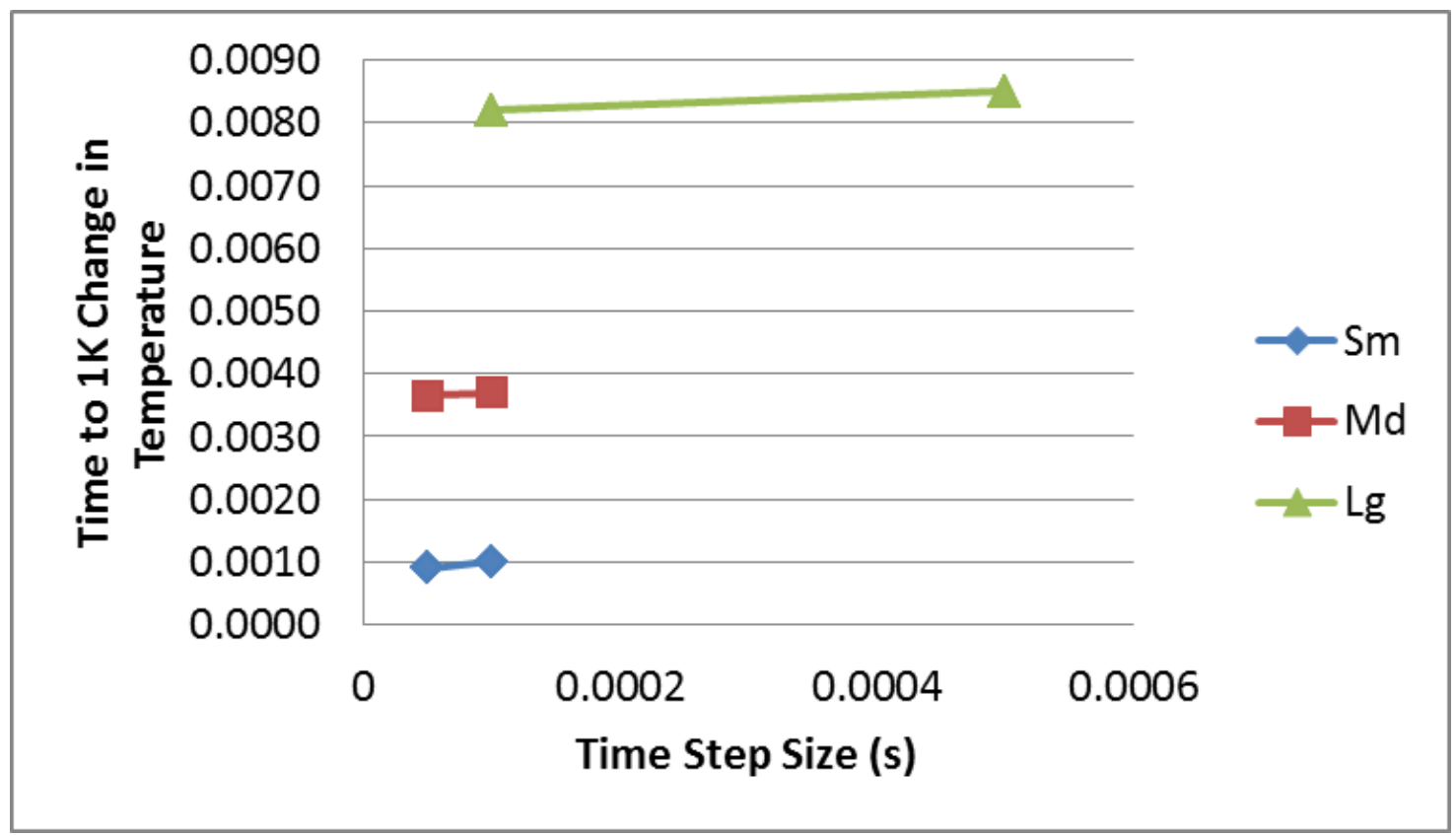

Figure 23: Model output vs. time step size for three particle diameters

The case of heating in the peak temperature zone is shown. In the figure, sm, md, and Ig refer to $300 \mu \mathrm{m}, 600 \mu \mathrm{m}$, and $900 \mu \mathrm{m}$ diameter particles respectively. These sizes correspond approximately with the range of expected particle diameters in the design space. The time step sizes were identical on the first run, at $0.0001 \mathrm{~s}$. After this, the time step size was altered to assess any change in output. The large size simulation transferred heat much slower than the other two, and the time step size was increased dramatically to decrease the time steps required to reach $1 \mathrm{~K}$ of fluid temperature difference. The other two cases used a reduced time step size on the following run using similar logic. The largest observed difference was around 3 $-4 \%$. This was deemed insignificant in this simulation. Going forward, this means that any of these time steps can be used.

The second check is for mesh scale. For the first run, the mesh was about 370,000 cells. The simulation was rerun with a much finer mesh, at over $1,000,000$ cells. A single case is shown for each in figure 24. 


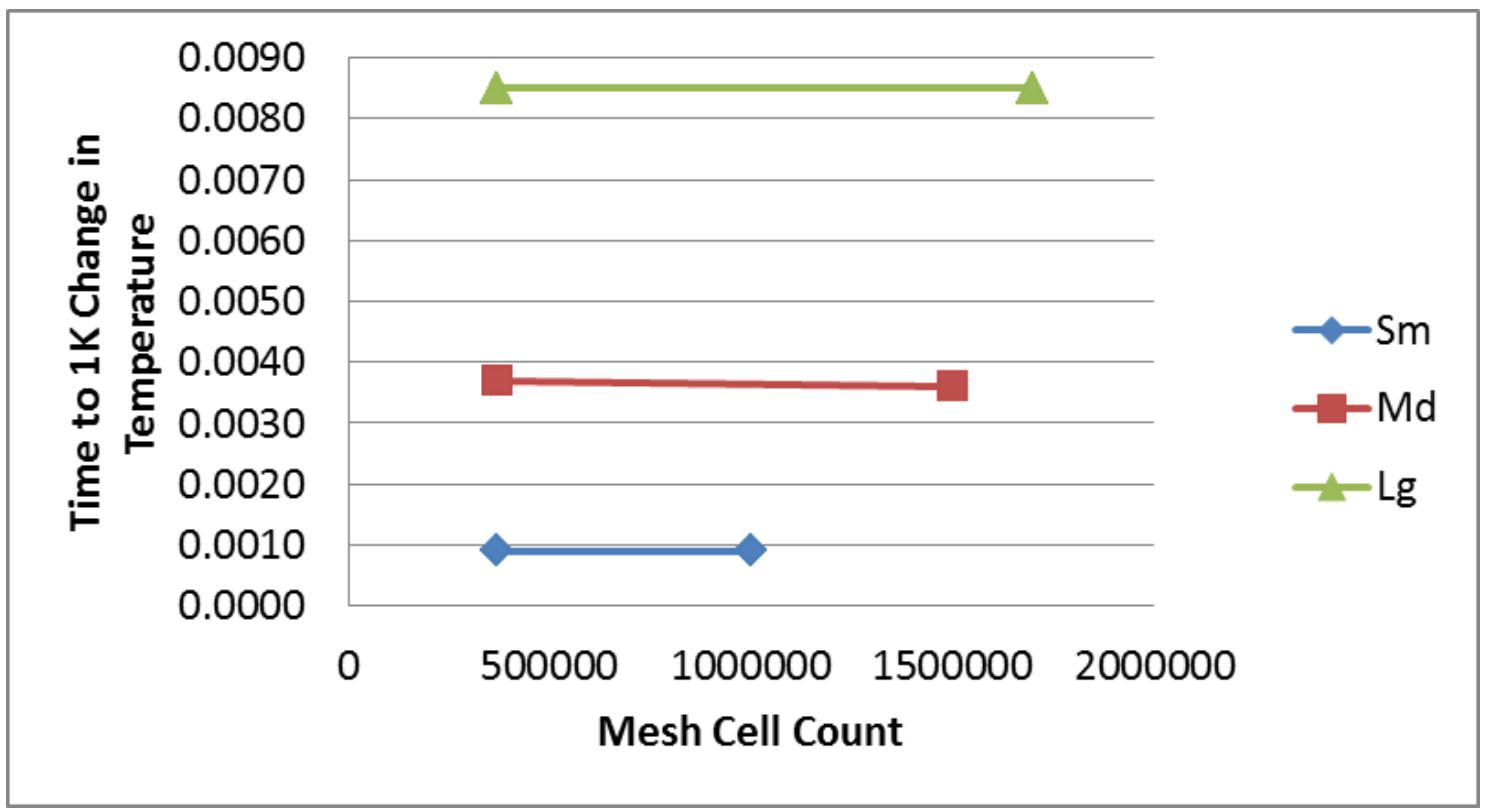

Figure 24: Model output vs. mesh size for three particle diameters

Again, resulting deviations in the output time value were negligible over the simulated range of mesh sizes. This means that the more compact model could be used going forward. The next check was for turbulence model independence. The initial runs were simulated with a laminar model (no turbulence factors). Turbulence generally increases mixing, so this could be a significant effect. The results between laminar and K-epsilon (enhanced wall treatment) turbulence models are depicted in figure 25 for each of the four run conditions.

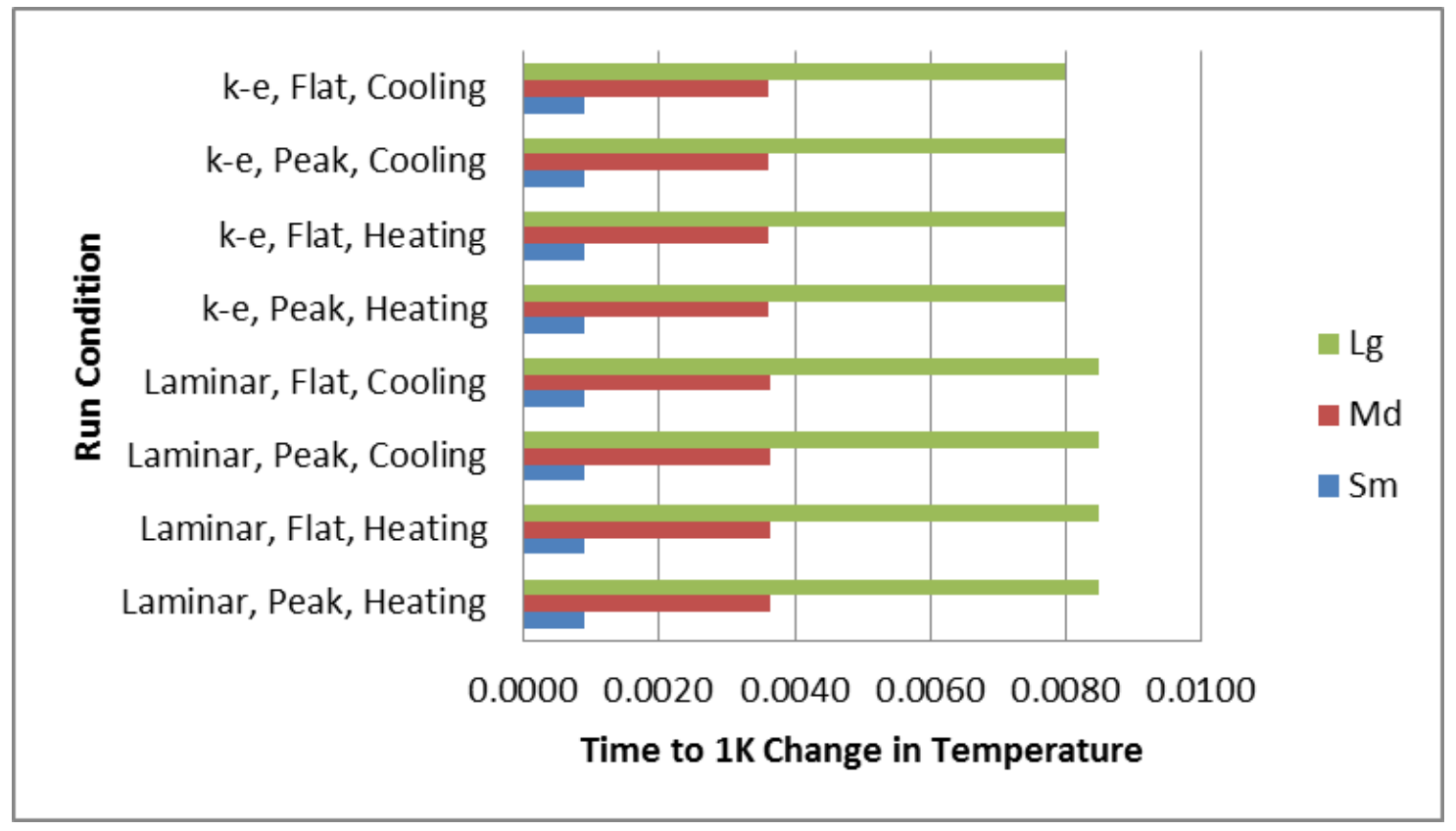

Figure 25: Turbulence model independence of model 
The turbulence model did affect the large-size model by about $5 \%$. Because the K-epsilon turbulence model did not increase the computation time significantly, it was used going forward to ensure that mixing is captured. Another take-away is that the differences between the four cases within each turbulence model group were negligible. This suggests that a single case properly represents the likely variations of starting temperature and heating versus cooling, even though material property data change significantly.

\subsection{3 - Fluid Velocity in Particle Void}

With model independence established a range of particle sizes was run. The hypothesis is that two regimes of heat transfer could span the particle size range of interest in this study. With the smallest sizes, the method of thermal equalization is primarily conduction. The time scale is such that convective currents aren't able to form before the driving temperature difference is negated. With the larger sizes, convection will become more and more prevalent. The bulk average velocity over time for the $300 \mu \mathrm{m}$ diameter particle case can be seen in figure 26.

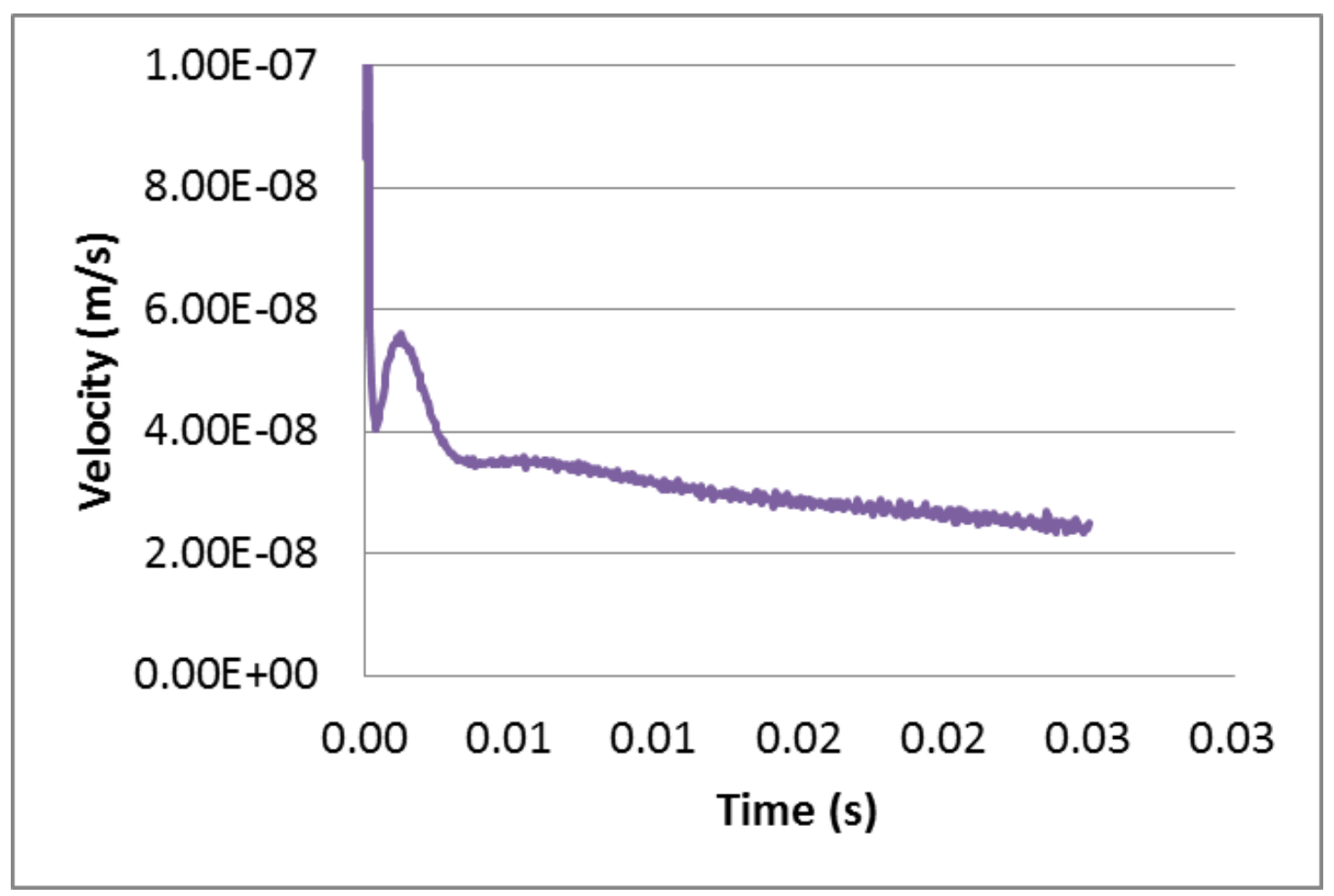

Figure 26: Average velocity vs. time for $300 \mu \mathrm{m}$ diameter particles

The initial velocity is high, which is an effect of the step-change in temperature discussed previously. This initial condition is artificial, and quickly reduced to realistic values. There is a small peak experienced near $0.005 \mathrm{~s}$, but it immediately begins to decay as the temperature difference decays. When the particle size is increased, this peak increases in size and breadth. The $900 \mu m$ particle results can be seen in figure 27 . 


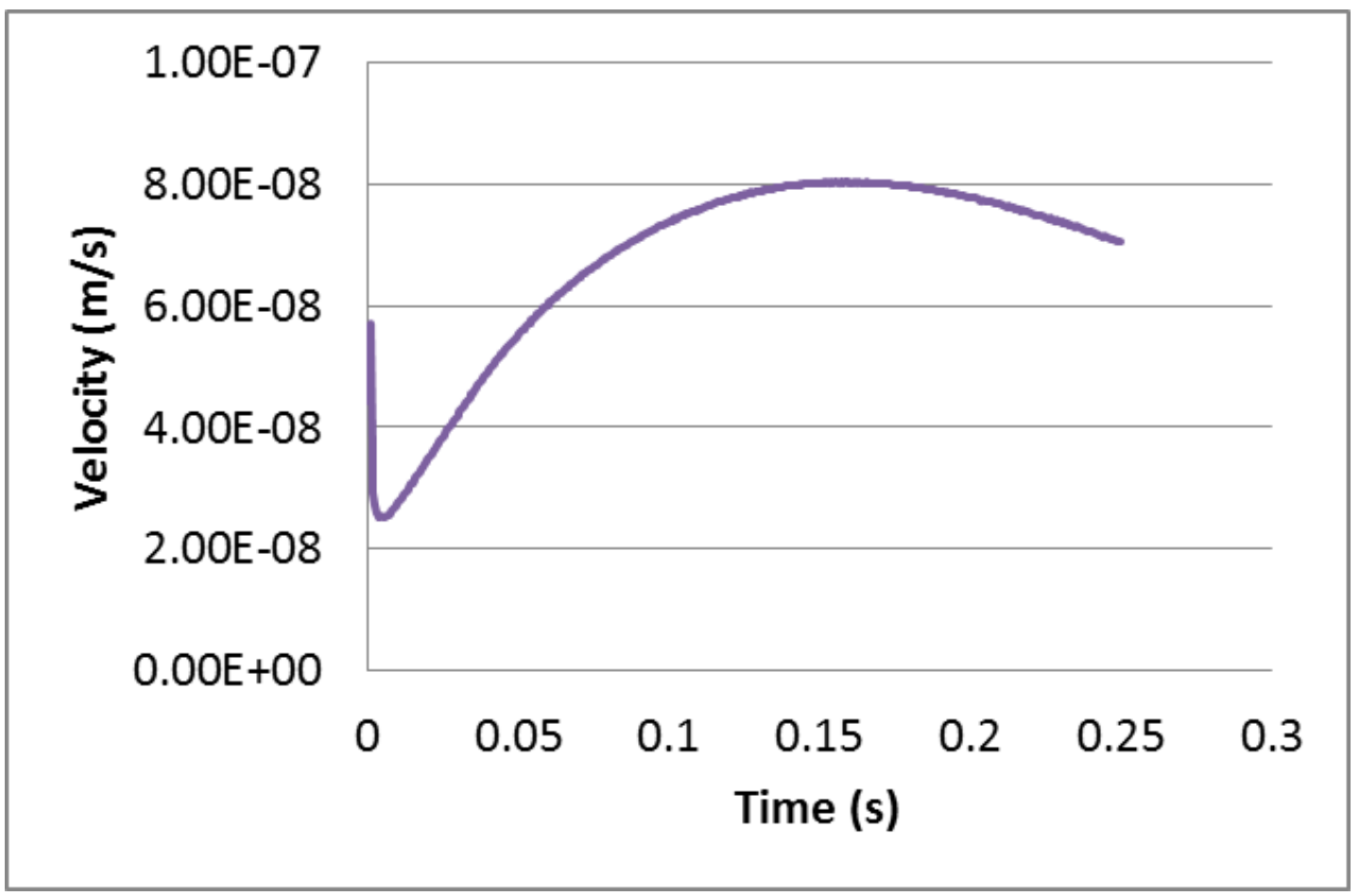

Figure 27: Average velocity vs. time for $900 \mu \mathrm{m}$ diameter particles

This simulation takes place over a much longer time span than the $300 \mu \mathrm{m}$ case, which allows a stronger current to form. The current only begins to decay toward the end of the simulation. Three larger sizes were simulated to further increase convection cell strength. These sizes all show convection current formation, with a basic toroid shape; shown in figure 28 for a $1800 \mu \mathrm{m}$ diameter void size. 


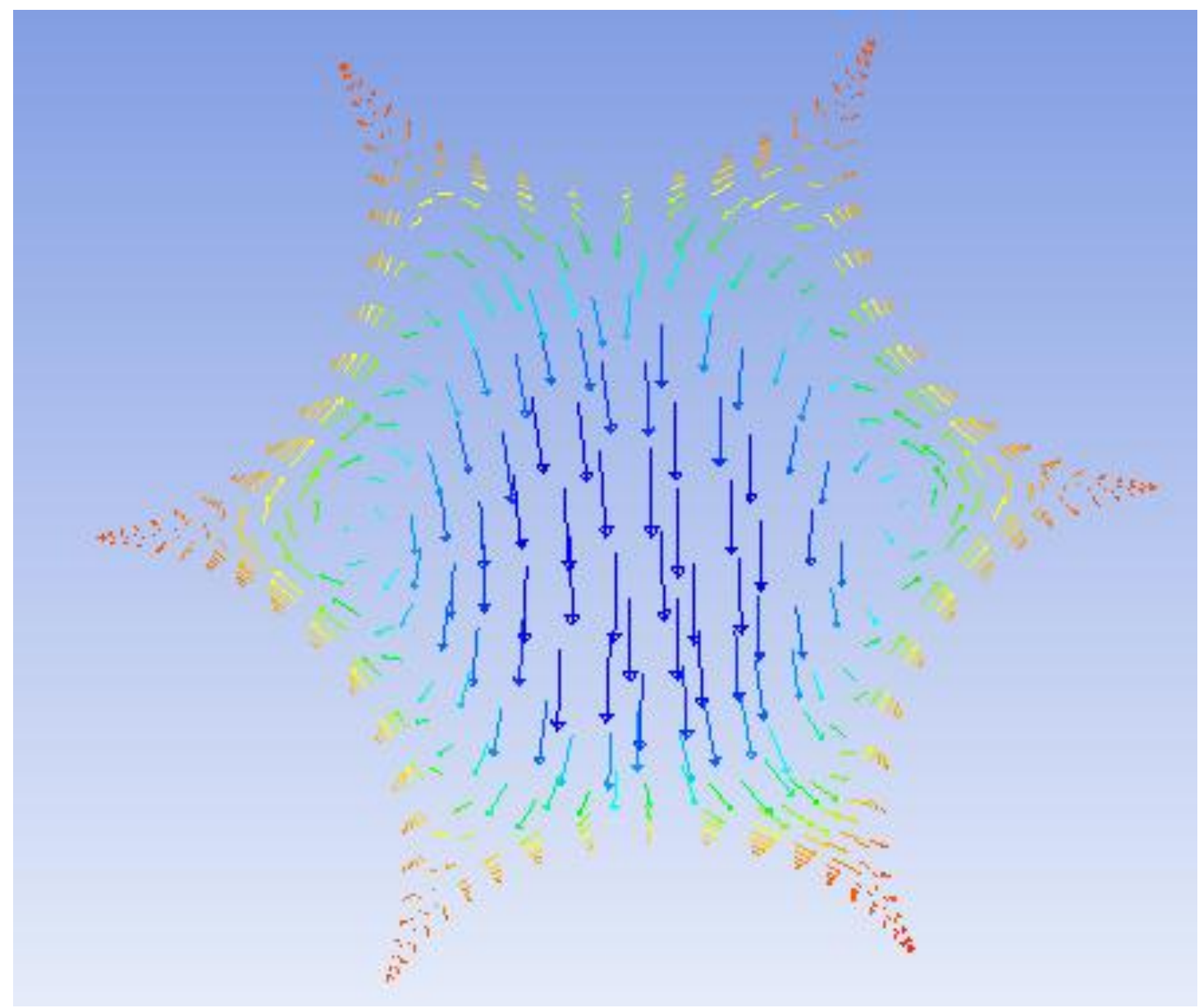

Figure 28: Convection cell cross-section in particle void

The arrows are colored by temperature, which has a range of $3 K$, with blue being the coldest. Fluid rises along the void edge and falls through the cool center of the void. The arrow length is scaled by velocity, which ranges from 0 to $1 \mathrm{E}-7 \mathrm{~m} / \mathrm{s}$. This low velocity suggests that the current is quite weak, and may not significantly influence heat transfer. In confirmation of this hypothesis, a path line through the convection circuit would take several days to cross completely through the void at the maximum rate. A particle diameter of $1800 \mu \mathrm{m}$ is much larger than the particles of interest in a physical machine, so it appears that pure conduction can be assumed for the design space.

Besides confirming the assumption of pure conduction this CFD study is useful for identifying the approximate time scale that the temperature change occurs on. This can be used for design guidance of the experimental apparatus. 


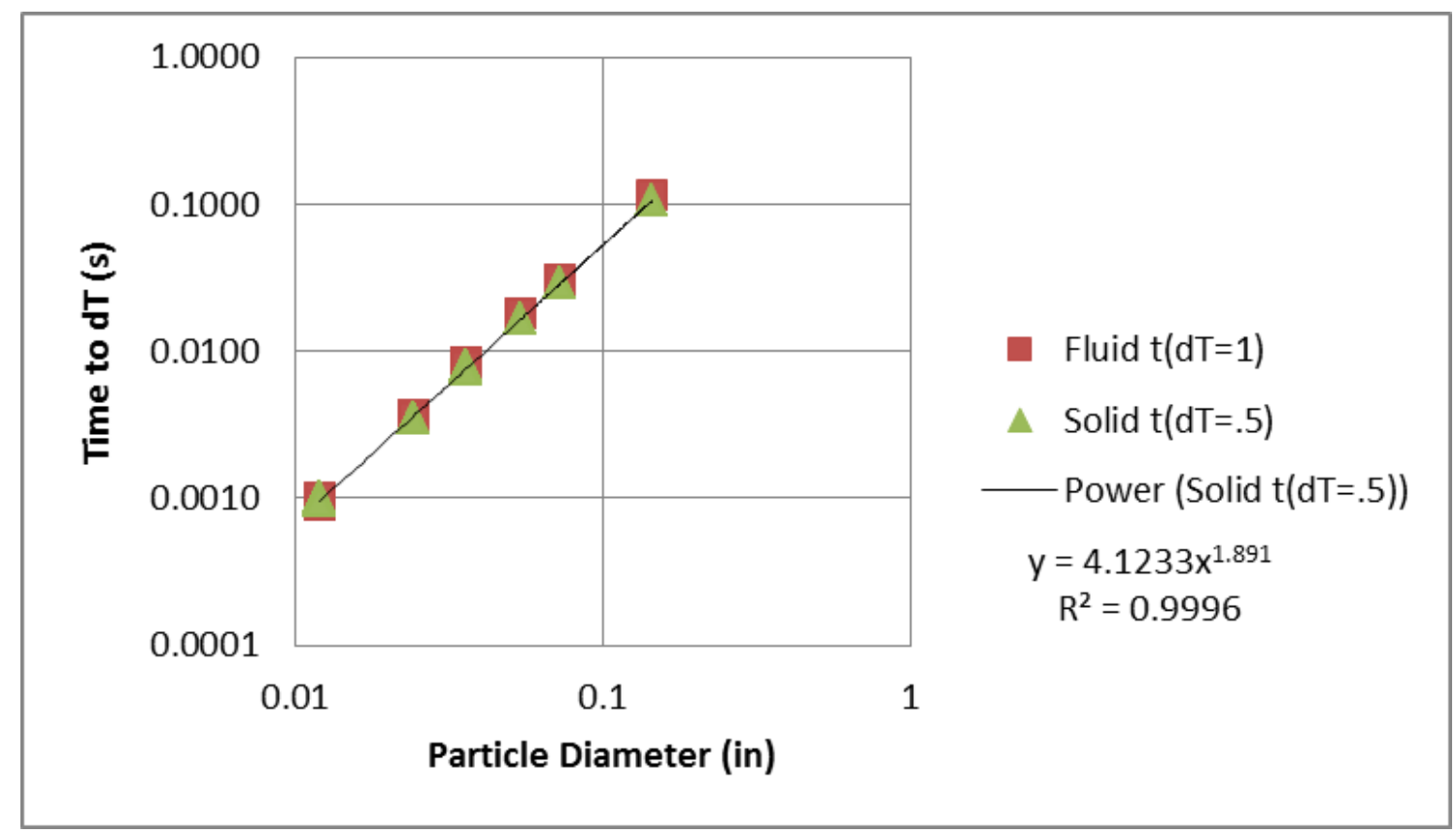

Figure 29: Particle diameter vs. Time to $1 \mathrm{~K}$ change in fluid temperature

Notice that the scale is log-log, and the data follows a power law. Figure 29 shows time to $1 \mathrm{~K}$ change in temperature for the fluid and $1 / 2 \mathrm{~K}$ change for the solid. They are almost identical because the thermal mass of the solid is double that of the fluid in these simulated cases. The main take-away from this plot is that a single trend can predict a very large range in particle sizes. In addition, the experiment must be able to capture trends from $0.001 \mathrm{~s}$ to $0.1 \mathrm{~s}$ in length.

\section{3 - Heat Transfer Model Development}

Knowing that pure conduction can be assumed, an analytical model can be developed to calculate heat transfer coefficient from material properties and geometry. This heat transfer coefficient can then be compared with experimental data to assess the validity of assumptions and simplifications.

\subsection{1 - Characteristic Length}

In order to simplify the problem a two lumped mass system was modelled. The lumped masses considered are the two phases contained in the regenerator, solid and fluid. The average internal resistance of each phase will be used to calculate a total series resistance. All temperatures used are bulk averages of either phase. 


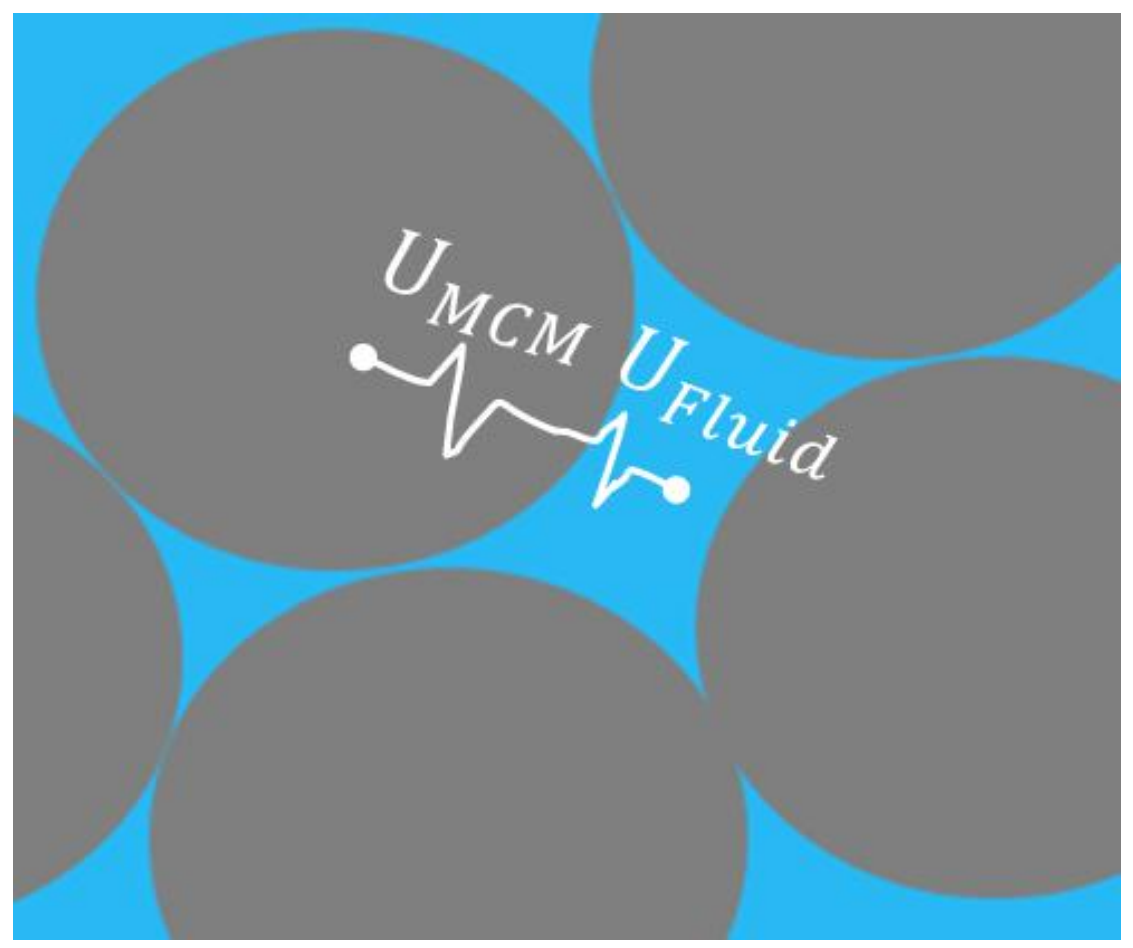

Figure 30: Thermal resistance network

In order to calculate thermal resistance a representative conduction length is needed. One approach to approximating a characteristic length for the fluid phase is to calculate the statistical void-size distribution which was studied by Alonso et al. (1995). They proposed and validated a function describing the void size distribution of randomly packed spheres using void fraction. The issue is that the function only includes voids larger than a hexagonal-close-pack interstitial void size. As this represents a large portion of the fluid volume, this work is not useful for this particular purpose. Additionally, this only applies to spherical particles, which is not the case.

A second approach for defining characteristic length is that of Engelbrecht (2005). By assuming even internal heat generation, an internal temperature gradient can be calculated. Using this gradient, the internal thermal resistance of the particle can be solved as a function of Biot number. The equivalent solid characteristic length for a sphere using this method is

$$
L_{c S}=\frac{D_{p}}{10}
$$

where $D_{p}$ is mean particle diameter (Using sieve hole sizes used in sorting) and $L_{c s}$ is solid phase characteristic length.

Although realistic for the spherical case, this method again neglects irregular geometry. A more general definition of characteristic length proposed by Incropera (2007) is

$$
L_{c}=\frac{\text { Volume }}{\text { Surface Area }} \text {. }
$$


where $L_{c}$ is generic characteristic length.

Given the irregular nature of the particles being studied, a variable is needed to adjust this ratio from known calculable geometric shapes. Sphericity is defined as the ratio of surface areas between a spherical particle and an irregular particle of equivalent volume (Wadell, 1935), or

$$
\theta=\frac{A_{\text {sphere }}}{A_{\text {particle }}}
$$

A sphericity of one indicates a perfect sphere, and sphericity drops from there as irregularity increases. As a point of reference, a regular tetrahedron has a sphericity of 0.671 . For solid particles, characteristic length can now be defined as

$$
L_{c s}=\frac{V_{\text {sphere }}}{A_{\text {particle }}}=\frac{\frac{1}{6} \pi D_{p}^{3}}{\frac{\pi D_{p}^{2}}{\theta}}=\frac{D_{p} \theta}{6} .
$$

where $V_{\text {sphere }}$ is volume of a sphere using particle diameter and $A_{\text {particle }}$ is surface area of a particle or surface area of a sphere divided by sphericity.

By the same method, the fluid phase characteristic length is

$$
L_{c f}=\frac{V_{f}}{A_{h x}}=\frac{D_{p \theta}}{6} \frac{\varepsilon}{(1-\varepsilon)} .
$$

where $V_{f}$ is volume of fluid phase per unit regenerator volume, $A_{h x}$ is surface area of the regenerator per unit regenerator volume (defined in 4.2.4 - Heat Transfer Area Calculations), $L_{c f}$ is fluid phase characteristic length, and $\varepsilon$ is regenerator void fraction (Fluid phase volume fraction)

When characteristic lengths for both phases (equations 17 and 18) are taken in series, the resulting heat transfer coefficient is

$$
U=\frac{1}{\left(\frac{L_{C S}}{k_{S}}+\frac{L_{c f}}{k_{f}}\right)}=\frac{1}{\left(\frac{D_{p} \theta}{6 k_{S}}+\frac{D_{p \theta} \varepsilon}{6(1-\varepsilon) k_{f}}\right)}
$$

where $\theta$ is sphericity (0 to 1 ), $D_{p}$ is mean particle diameter (Using sieve hole sizes used in sorting), $\varepsilon$ is void fraction in regenerator packing, $k_{s}$ is solid material conductivity, and $k_{f}$ is fluid material conductivity. 


\subsection{2 - Validity of an Exponential Model}

The next step is to calculate typical Biot numbers for each lumped mass, to determine whether internal thermal resistance can be neglected for either phase within the expected design space. Typically, Biot number is calculated as

$$
B i=\frac{h L_{c}}{k} \text {. }
$$

where $h$ is generic convection coefficient for the exterior of a thermal mass, $L_{c}$ is generic characteristic length for the thermal mass shape, $k$ is generic conduction coefficient for the interior of the thermal mass.

Since the case of interest includes two conductive components, the convection coefficient can be traded for an additional conductivity and characteristic length. A range of expected Biot numbers can be calculated for both the solid and fluid phases using the previously establish characteristic lengths as

$$
B i_{s}=\frac{k_{f} L_{c s}}{k_{s} L_{c f}} \quad \text { and } \quad B i_{f}=\frac{k_{s} L_{c f}}{k_{f} L_{c s}}
$$

where $k_{f}$ is fluid phase conductivity and $k_{s}$ is solid phase conductivity.

Typically, a resistance component with a Biot number less than 0.1 is considered negligible. In other words, the assumption of all mass being lumped at a geometric node with a basic constant thermal resistance is valid. In order to calculate Biot numbers, the design space must be defined. The available particle diameters range from 300-1250 $\mu \mathrm{m}$. The working fluid conductivity ranges from pure water to pure ethylene glycol, or $0.563-0.258 \frac{\mathrm{W}}{\mathrm{mK}}$ respectively. Solid conductivity for the MCM ranges from 2.5-7.5 $\frac{\mathrm{W}}{\mathrm{mK}}$ (Legait et al., 2014; Liu et al., 2012; Fujieda, 2004).

The range of Biot number values coming from all possible combinations of variables is 0.01-87, meaning certain cases will contain a negligible internal resistance component. For consistency all cases will include both components. Using the previously calculated thermal resistance between phases, a time stepping model can readily calculate energy transfer as a function of time during a dwell period. A time stepping model requires a large number of calculations to reach a solution. Ideally, a closed form solution can be found in order to reduce computation time.

An infinite series of terms can be used to calculate temperature versus time in a case with significant internal resistance within a thermal mass. This is the case for any of the parameter sets yielding a Biot number greater than 0.1. Even further simplifications of this model can be made in specific circumstances. 
Fourier number,

$$
F O=\frac{\alpha t}{L_{c}^{2}}
$$

where $\alpha$ is thermal diffusivity of the thermal mass and $t$ is heat transfer time.

is dimensionless heat transfer time. A single exponential term can be used to approximate the infinite series of terms if Fourier number is greater than 0.2 (Incropera, 2007). What this means qualitatively is that initially thermal resistance is highly variable because temperature gradients are being established spatially. The resistance then settles on a nearly constant value for the remainder of the transition to thermal equilibrium.

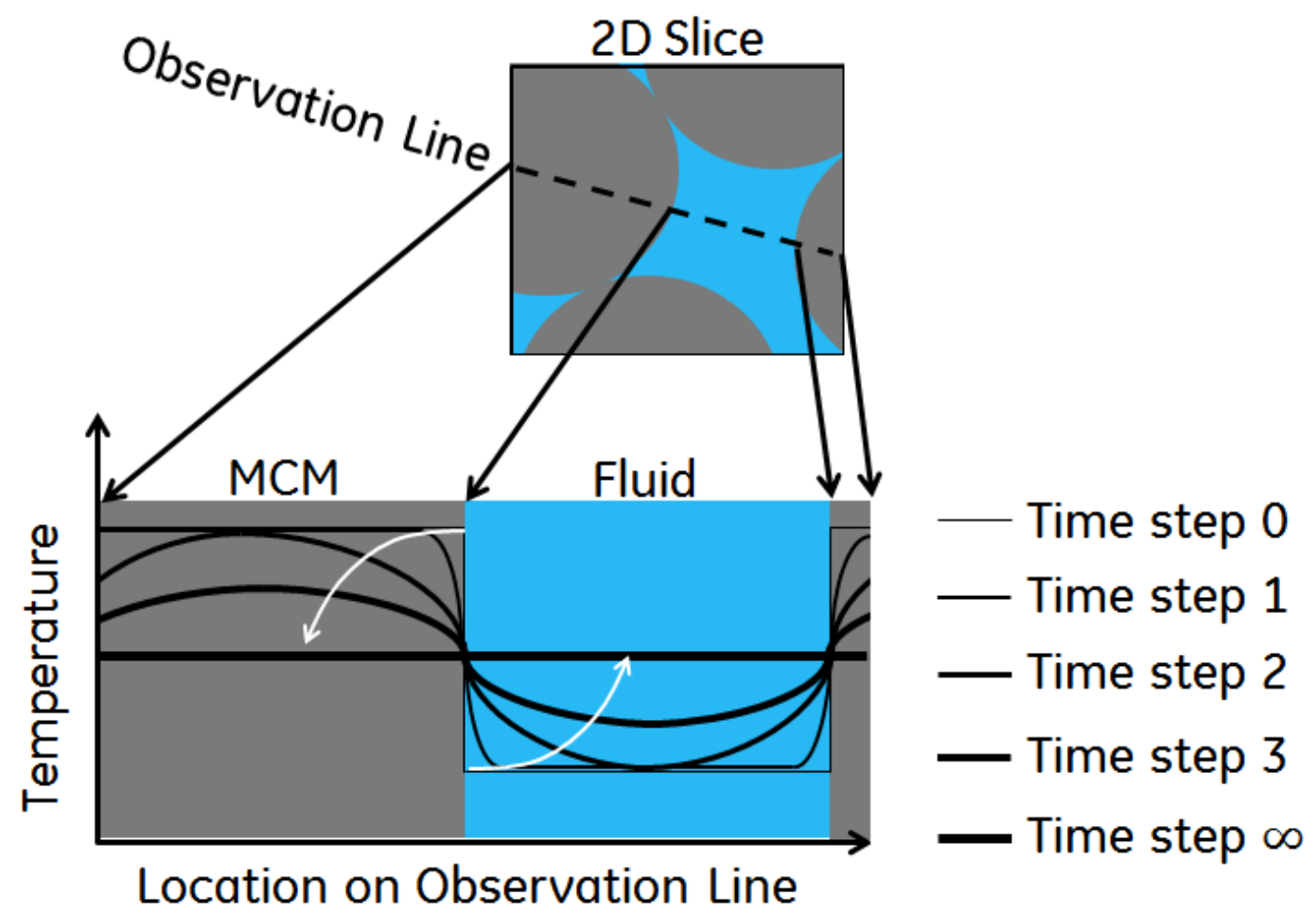

Figure 31: Spacial and temporal temperature distribution following a step change in MCM temperature

The spacial temperature distribution is shown in figure 31 at arbitrary points in time following a step change in temperature of the MCM. The initial heat transfer rate is extremely high, due to the infinite slope of the temperature distribution at the solid-fluid interface. This is a product of Fourier's law of conduction. A temperature difference with zero conduction length results in an infinite heat flux. What this means in reality is that this condition never truly exists if the time and space scales are small enough.

Until the spacial temperature distribution is fully established (time step 2, figure 31), the observed thermal resistance will be highly variable. This is the product of a changing conduction 
length. Beyond time step 2 in figure 31, conduction distance is effectively constant, and thermal resistance can also be approximated as constant. This is qualitatively when Fourier number is large, and a single exponential term approximation can be used in lieu of an infinite series.

Going forward, the assumption is made that spacial temperature distributions are developed during the magnetization period, and any measurements made after this period can reasonably use the single term approximation. This becomes especially import in the Heat Transfer Experiments chapter, where the initial heat transfer rates were not observed.

\subsection{3 - Exponential Behavior of a Coupled Two Mass System}

If a single term exponential approximation is to be used, a time constant must be selected. In a typical single mass quenching problem, time constant is defined as

$$
\tau_{c \text { quench }}=\frac{m C_{p}}{U A}
$$

where $\tau_{c \text { quench }}$ is generic time constant for a lumped mass quenching problem, $m$ is mass of lumped mass, $C_{p}$ is heat capacity of lumped mass material, $U$ is heat transfer coefficient, and $A$ is heat transfer area.

This definition cannot be used in this case, as there are two thermal masses, and therefore two different time constant possibilities. The system time constant needs to be calculated assuming that the two masses are coupled. The time constant of this coupled two mass system will be considered an unknown for the time being. The overall temperature profile of the coupled solid phase is defined as

$$
T_{s}(t)=T_{\infty}+\left(T_{i s}-T_{\infty}\right) \exp \left(\frac{-t}{\tau_{c s}}\right)
$$

where $T_{S}(t)$ is thermal mass average solid temperature as a function of time, $T_{i s}$ is initial temperature of the solid thermal mass, $T_{\infty}$ is final temperature of thermal mass (Infinite time has passed), $t$ is heat transfer time, and $\tau_{c s}$ is solid phase time constant.

Likewise, the temperature profile of the fluid phase is define as

$$
T_{f}(t)=T_{\infty}+\left(T_{i f}-T_{\infty}\right) \exp \left(\frac{-t}{\tau_{c f}}\right) .
$$

where $T_{f}(t)$ is thermal mass average fluid temperature as a function of time, $T_{i f}$ is initial temperature of the fluid thermal mass, and $\tau_{c f}$ is fluid phase time constant.

The form of the desired exponential functions is shown in figure 32 . 


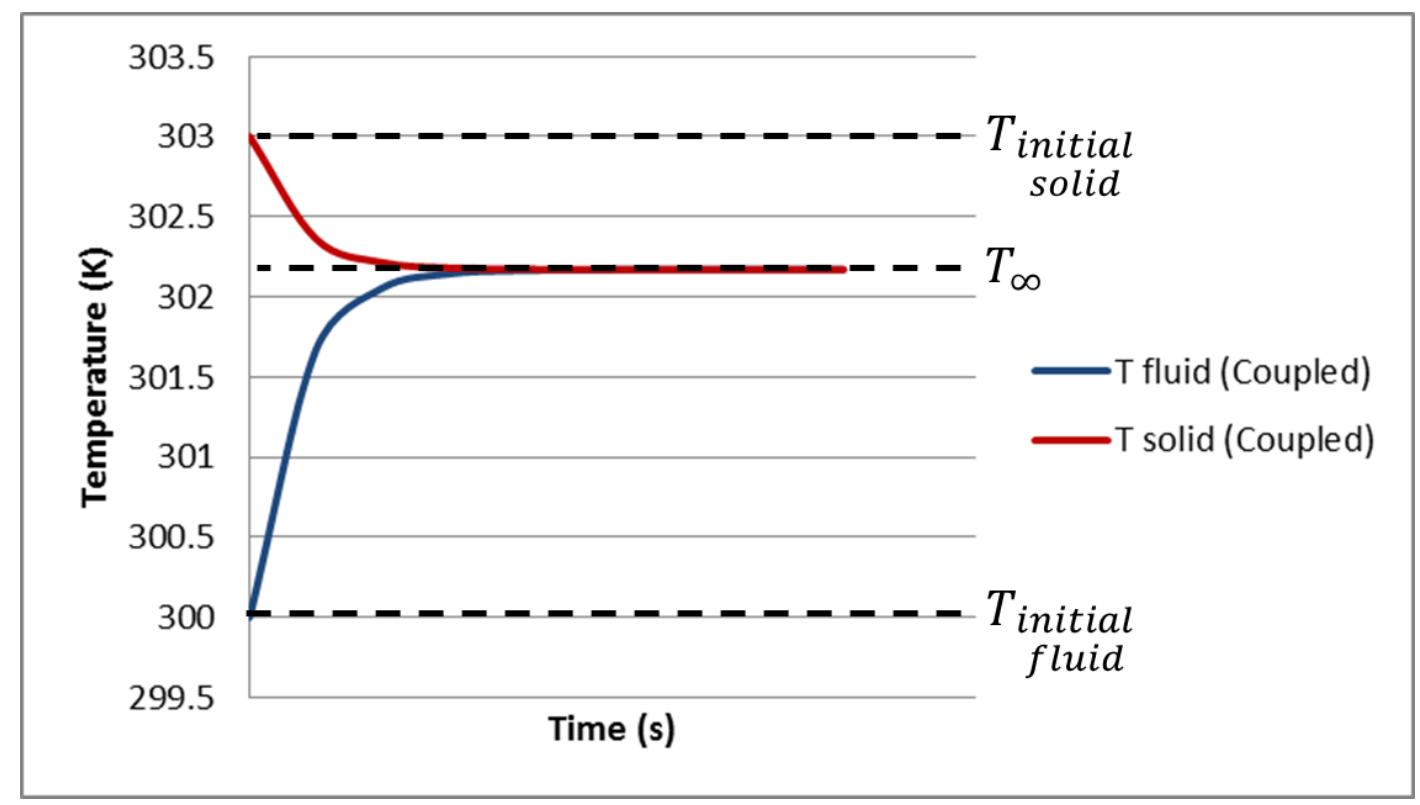

Figure 32: Exponential model of temperature change for two coupled masses

In this function the time constant is unknown. However, initial temperatures are known and the final temperature can be calculated using energy conservation. Thermal energy per unit volume of regenerator contained in the fluid phase at $t=0$ is

$$
\underset{\text { fluid } t=0}{E_{\text {thermal }}}=\varepsilon\left(\rho C_{p}\right)_{f} T_{i f}
$$

where $\rho_{f}$ is fluid phase material density and $C_{p f}$ is fluid phase material heat capacity.

Likewise, energy contained in the solid phase at $\mathrm{t}=0$ is

$$
\underset{\text { solid } t=0}{E_{\text {thermal }}}=(1-\varepsilon)\left(\rho C_{p}\right)_{s} T_{i s}
$$

where $\rho_{s}$ is solid phase material density and $C_{p s}$ is solid phase material heat capacity.

Thermal energy per unit volume of regenerator contained by the system at equilibrium is

$$
\underset{\text { sys } \infty}{E_{\text {shermal }}}=\left((1-\varepsilon)\left(\rho C_{p}\right)_{s}+\varepsilon\left(\rho C_{p}\right)_{f}\right) T_{\infty}
$$

With no heat leak, conservation of energy dictates that

$$
\underset{\text { sys } t=0}{E_{\text {thermal }}}=\underset{\text { sys } \infty}{E_{\text {thermal }}}=E_{\text {fluid } t=0}^{\text {fhermal }}+E_{\text {solid } t=0}
$$

Substituting and rearranging equations 26,27 , and 28 into equation 29 yields 


$$
T_{\infty}=\frac{\varepsilon\left(\rho C_{p}\right)_{f} T_{i f}+(1-\varepsilon)\left(\rho C_{p}\right)_{s} T_{i s}}{(1-\varepsilon)\left(\rho C_{p}\right)_{s}+\varepsilon\left(\rho C_{p}\right)_{f}} .
$$

This leaves only time constants of equations 24 and 25 as unknown. These cannot be calculated using the typical lumped mass quenching definition found in equation 23 . In order to find these time constants the most basic qualitative definition of time constant must be evaluated. It is a value in an exponential function which corresponds to the rate at which the function converges. In fact it is the only parameter corresponding to rate in an exponential function, meaning a single boundary condition can be used to find its value for each curve.

Knowing the initial conditions, initial heat transfer rate is also known. This can be used as the boundary condition to determine time constant. This rate is defined by another set of exponential functions which come from a case of uncoupled single masses. If each mass were quenched individually using a constant opposing temperature, the curves would like those in figure 33.

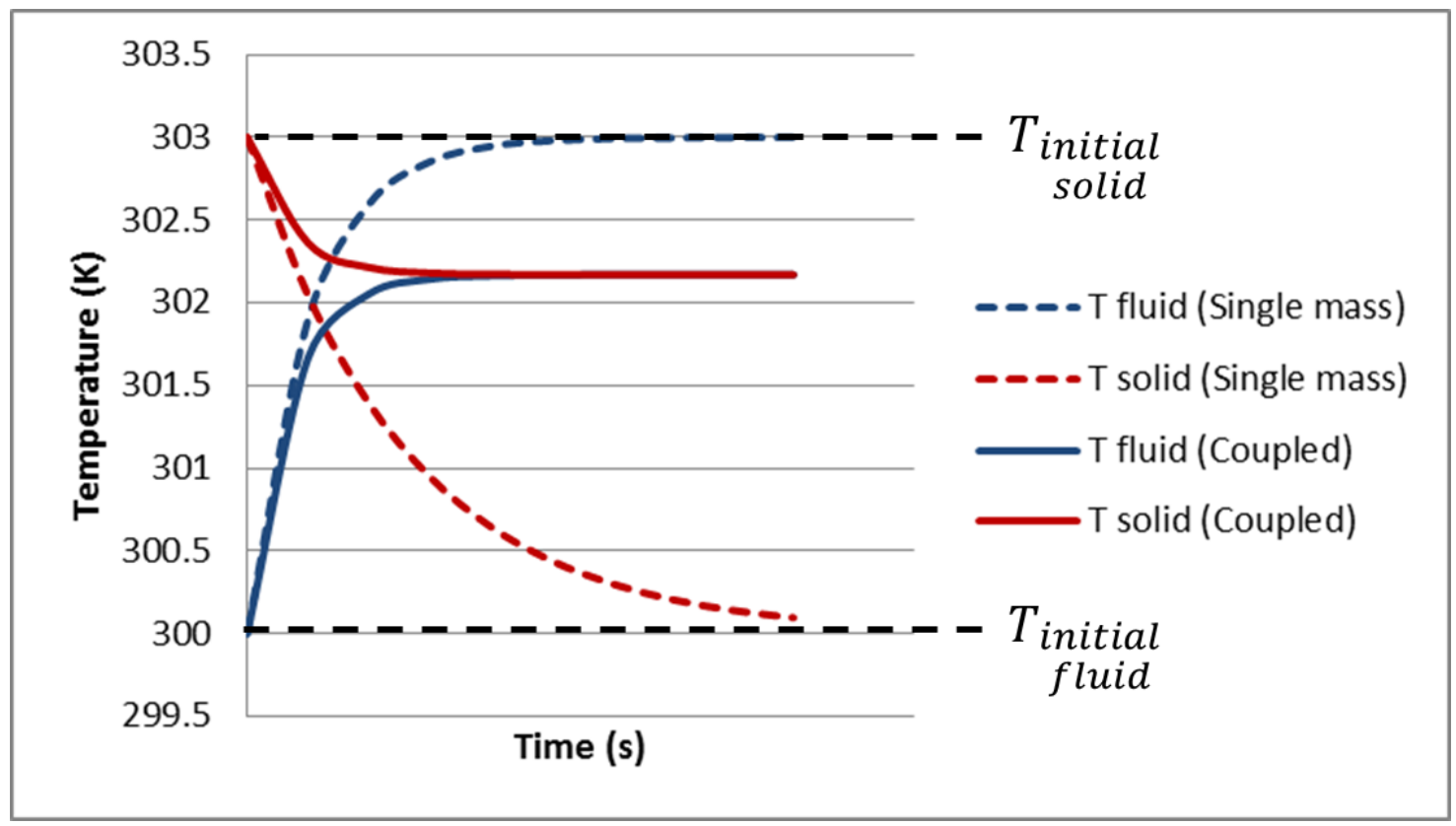

Figure 33: Exponential model of temperature change for independent and coupled masses

The uncoupled single mass curves both converge toward the opposing initial temperature. Although the initial slopes are correct, the curves cross after some time. This is because the function assumes the opposing temperature to be constant. The time constants used for each uncoupled equation use the same heat transfer coefficient and area, but different thermal masses. The fluid initial (single uncoupled mass) time constant is

$$
\tau_{c i f}=\frac{\varepsilon\left(\rho C_{p}\right)_{f}}{U A_{h x}} .
$$


Likewise, the solid phase initial (uncoupled) time constant is

$$
\tau_{c i s}=\frac{(1-\varepsilon)\left(\rho C_{p}\right)_{s}}{U A_{h x}} .
$$

The only valid portion of this uncoupled profile is the initial heat transfer rate, or slope. Using the time constants in equations 31 and 32 for each individual mass, the initial (single uncoupled mass) functions shown in figure 33 are

$$
T_{i s}(t)=T_{\infty}+\left(T_{i s}-T_{\infty}\right) \exp \left(\frac{-t}{\tau_{c i s}}\right)
$$

and

$$
T_{i f}(t)=T_{\infty}+\left(T_{i f}-T_{\infty}\right) \exp \left(\frac{-t}{\tau_{c i f}}\right)
$$

The derivative of equation 34 is

$$
T_{i f}^{\prime}(t)=-\left(\frac{T_{i f}-T_{i s}}{\tau_{c i f}}\right) \exp \left(\frac{-t}{\tau_{c i f}}\right)
$$

Likewise, the solid phase (equation 33) derivative is

$$
T_{i s}^{\prime}(t)=-\left(\frac{T_{i s}-T_{i f}}{\tau_{c i s}}\right) \exp \left(\frac{-t}{\tau_{c i s}}\right) .
$$

At $t=0$, these become

$$
T_{i f}^{\prime}(t)=-\left(\frac{T_{i f}-T_{i s}}{\tau_{c i f}}\right)
$$

and

$$
T_{i s}^{\prime}(t)=-\left(\frac{T_{i s}-T_{i f}}{\tau_{c i s}}\right)
$$

The derivative of the coupled solid function (equation 24) is

$$
T_{s}^{\prime}(t)=-\left(\frac{T_{i s}-T_{\infty}}{\tau_{c}}\right)
$$

Likewise, the derivative of the coupled fluid function (equation 25) is

$$
T_{f}^{\prime}(t)=-\left(\frac{T_{i f}-T_{\infty}}{\tau_{c}}\right) .
$$

Setting equations 38 and 39 to be equal and rearranging yields 


$$
\tau_{c S}=\tau_{c i s}\left(\frac{T_{i s}-T_{\infty}}{T_{i s}-T_{i f}}\right) .
$$

Likewise, setting equations 37 and 40 to be equal yields

$$
\tau_{c f}=\tau_{c i f}\left(\frac{T_{i f}-T_{\infty}}{T_{i f}-T_{i s}}\right),
$$

This is the time constant of a coupled two mass system using known initial and final temperature points. It can also be expressed in terms of thermal masses of the two phases. Manipulating equation 30,

$$
\left(\frac{T_{i f}-T_{\infty}}{T_{i f}-T_{i s}}\right)=\frac{\varepsilon\left(\rho C_{p}\right)_{f}}{\left(\varepsilon\left(\rho C_{p}\right)_{f}+(1-\varepsilon)\left(\rho C_{p}\right)_{f}\right)} .
$$

The left-hand side of the equation is identical to the time constant multiplier shown in equation 42. It can be manipulated in a similar way to match equation 41 . This means that time constants can be redefined by thermal masses, as

$$
\tau_{c s}=\tau_{c i s} \frac{\varepsilon\left(\rho C_{p}\right)_{f}}{\left(\varepsilon\left(\rho C_{p}\right)_{f}+(1-\varepsilon)\left(\rho C_{p}\right)_{s}\right)}
$$

and

$$
\tau_{c f}=\tau_{c i f} \frac{(1-\varepsilon)\left(\rho C_{p}\right)_{s}}{\left(\varepsilon\left(\rho C_{p}\right)_{f}+(1-\varepsilon)\left(\rho C_{p}\right)_{s}\right)} .
$$

Furthermore, conservation of energy dictates that the two coupled masses must converge at the same rate, or

$$
\tau_{c s}=\tau_{c f}=\tau_{c \text { coupled }} .
$$

As proof of this method this equality holds true; equations 44 and 45 are equal with equations 31 and 32 substituted in. From equation 24, the final exponential function for the solid phase is

$$
T_{S}(t)=T_{\infty}+\left(T_{i s}-T_{\infty}\right) \exp \left(\frac{-t}{\frac{\varepsilon\left(\rho C_{p}\right)_{f}}{U A_{h x}} \frac{(1-\varepsilon)\left(\rho C_{p}\right)_{s}}{\left(\varepsilon\left(\rho C_{p}\right)_{f}+(1-\varepsilon)\left(\rho C_{p}\right)_{s}\right)}}\right)
$$

From equation 25 , the corresponding fluid phase function is 


$$
T_{f}(t)=T_{\infty}+\left(T_{i f}-T_{\infty}\right) \exp \left(\frac{-t}{\frac{\varepsilon\left(\rho C_{p}\right)_{f}}{U A_{h x}} \frac{(1-\varepsilon)\left(\rho C_{p}\right)_{s}}{\left(\varepsilon\left(\rho C_{p}\right)_{f}+(1-\varepsilon)\left(\rho C_{p}\right)_{s}\right)}}\right)
$$

Recall that the basic function for time constant in a thermal quenching problem is

$$
\tau_{c \text { quench }}=\frac{m C_{p}}{U A} .
$$

From this, time constant can be described as a thermal mass $\left(m C_{p}\right)$ multiplied by a thermal resistance $(1 / U A)$. The time constant from equation 46 is

$$
\tau_{c \text { coupled }}=\frac{1}{U A_{h x}} \frac{\varepsilon\left(\rho C_{p}\right)_{f}(1-\varepsilon)\left(\rho C_{p}\right)_{s}}{\left(\varepsilon\left(\rho C_{p}\right)_{f}+(1-\varepsilon)\left(\rho C_{p}\right)_{s}\right)} .
$$

From equation 49 , the equivalent thermal mass of a coupled system is

$$
\left(m C_{p}\right)_{s y s}=\frac{\left(\varepsilon\left(\rho C_{p}\right)_{f}\right)\left((1-\varepsilon)\left(\rho C_{p}\right)_{s}\right)}{\left(\varepsilon\left(\rho C_{p}\right)_{f}+(1-\varepsilon)\left(\rho C_{p}\right)_{f}\right)}
$$

Another way of expressing this is

$$
\left(m C_{p}\right)_{s y s}=\frac{1}{\left(\frac{1}{\varepsilon\left(\rho C_{p}\right)_{f}}+\frac{1}{(1-\varepsilon)\left(\rho C_{p}\right)_{f}}\right)}=\frac{1}{\left(\frac{1}{\left(m C_{p}\right)_{f}}+\frac{1}{\left(m C_{p}\right)_{s}}\right)} .
$$

The form is that of a series of a two thermal masses in a heat transfer network. This also illustrates similarities with electrical circuits, where this system would be represented as two capacitors (storage), and one resistor (resistance). Subjecting a capacitor to a step change in charge is equivalent to creating a step change in temperature of one thermal mass. 

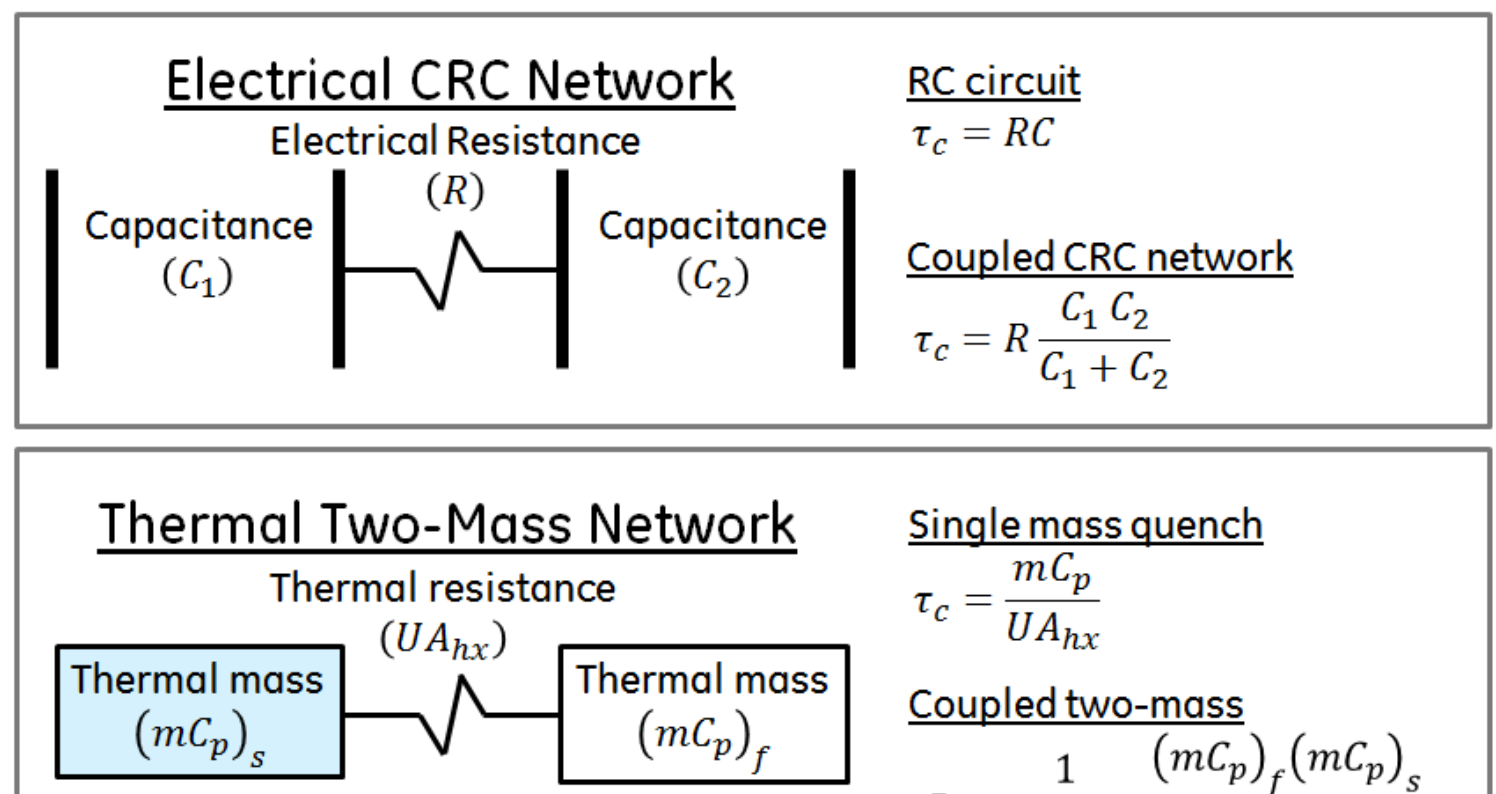

Single mass quench

$\tau_{c}=\frac{m C_{p}}{U A_{h x}}$

\section{Coupled two-mass}

$\tau_{c}=\frac{1}{U A_{h x}} \frac{\left(m C_{p}\right)_{f}\left(m C_{p}\right)_{s}}{\left(m C_{f}+\left(m C_{p}\right)_{s}\right.}$

Figure 34: Time constants for equivalent thermal and electrical networks.

The time constant of an electrical system can be defined by equivalent electrical impedance, whereas a thermal system time constant is defined by equivalent thermal impedance. As an additional proof, this function is compared with the previously described time stepping model. The results are shown in figure 35 for the fluid temperature using identical properties and constant UA. 


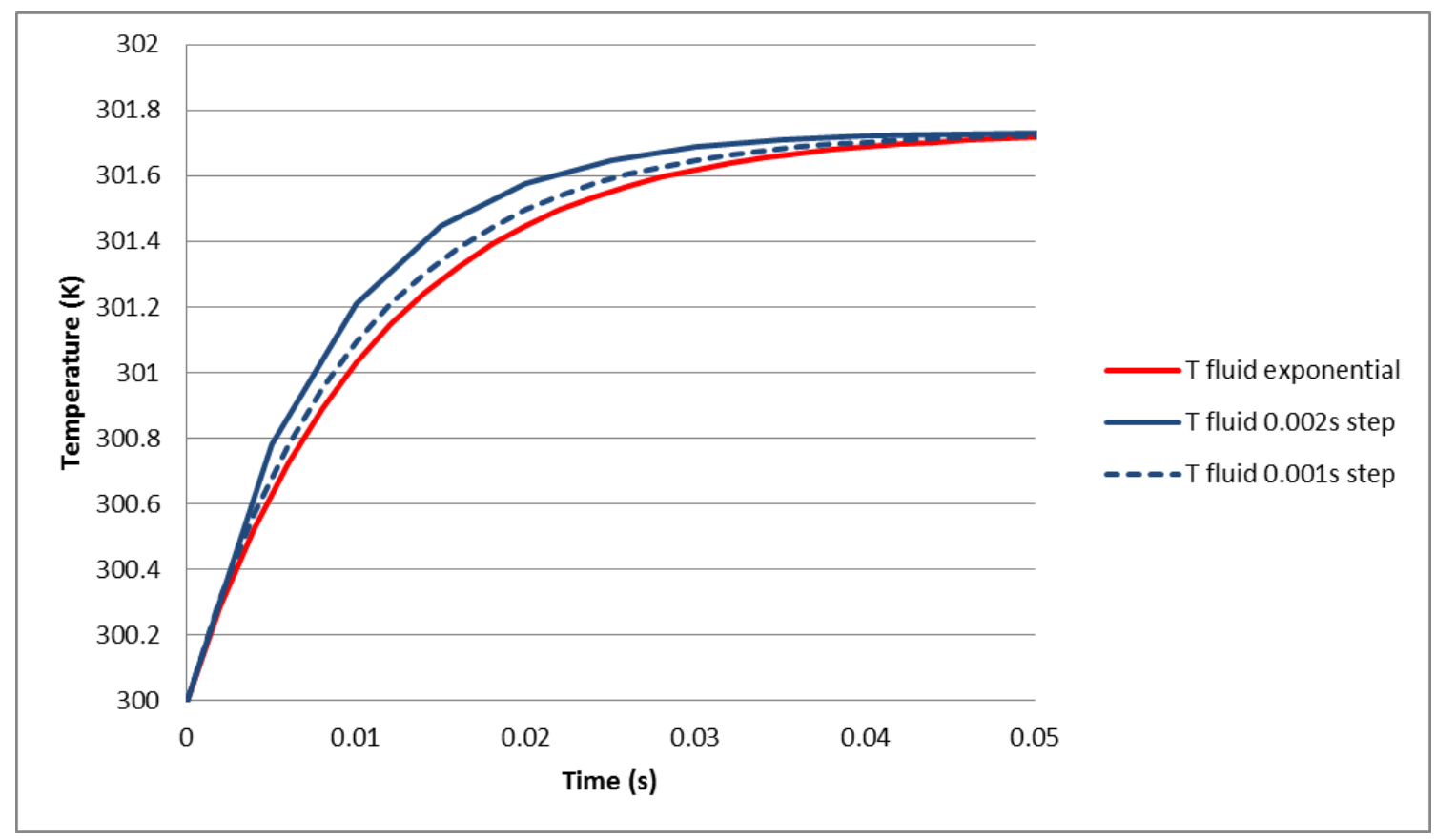

Figure 35: Exponential vs. time stepping model for fluid temperature change over time

As time step size is reduced the models match more and more closely, which validates the exponential two-mass methodology. In this particular case a series of 50 calculations is replaced by a single calculation, showing the utility of a coupled exponential model. Coupled exponential methodology can also be used in reverse, to calculate a UA value from an exponential curve fitted to measured data. The method will be applied in the Heat Transfer Experiment chapter.

\subsection{4 - Heat Transfer Area Calculations}

The final piece which has not yet been discussed is heat transfer area, or the surface area of the regenerator. Area will be calculated on a per regenerator volume basis, just as thermal mass was calculated previously. The approach is to use void fraction to calculate the number of particles per unit regenerator volume,

$$
N_{\substack{\text { particles } \\ \text { volume } \\ \text { volume }}}=\frac{V_{\text {solid }}}{V_{\text {particle }}}=\frac{(1-\varepsilon)}{\frac{\pi}{6} D_{p}^{3}} .
$$

Surface area for an individual particle,

$$
A_{\text {particle }}=\frac{\pi D_{p}^{2}}{\theta}
$$

is found using sphericity and diameter. Finally, regenerator surface area per unit volume is found by combining the equations 52 and 53 , 


$$
A_{h x}=A_{\text {particle }} N_{\begin{array}{c}
\text { particles } \\
\text { per regen } \\
\text { volume }
\end{array}}=\frac{6(1-\varepsilon)}{D_{p} \theta} .
$$

Diameter, void fraction, and sphericity are the only parameters required to calculate surface area per unit volume of a regenerator. In reality, diameter is controlled by screen sorting of particles. Void fraction is measurable using fluid displacement or packing weight and density. In order to find sphericity a more involved experiment must be performed using pressure drop. 


\section{4 - EXPERIMENTAL WORK}

\section{1 - Experimental Determination of Sphericity}

When a time constant is observed experimentally, there are three main driving components: thermal mass, heat transfer area, and heat transfer coefficient. Of these, thermal mass is known from material properties and the other two are unknown. In order to independently determine heat transfer coefficient, heat transfer area must be measured separately.

\subsection{1 - Methodology for Determining Sphericity Experimentally}

Using a method created by Ergun (1952), surface area can be calculated from pressure loss. All parameters except for sphericity are known in the surface area calculation, meaning sphericity can be determined from pressure drop.

Fundamentally, pressure loss is a product of viscous and inertial components. The viscous component should be most prevalent at lower Reynolds numbers, being driven by shear forces. The shear is between the bulk fluid velocity and the static solid phase. The viscous resistance is directly dependent on surface area of the solid phase. The inertial component will prevail at higher Reynolds numbers, being driven by turbulence and boundary layer separation. Reynolds number for a packed bed in this context is defined as

$$
R e=\frac{D_{p} v_{s f} \rho_{f}}{(1-\varepsilon) \mu_{f}} .
$$

where $R e$ is Reynolds number, $v_{s f}$ is superficial fluid velocity (fluid velocity assuming an unpacked container at the given flow rate), and $\mu_{f}$ is fluid dynamic viscosity.

The expected Reynolds numbers are well within the laminar regime for this study. Many pressure drop calculations are based on work done by Ergun and Orning (1949). Their original correlation is based around experimentation on spherical particles. Pressure drop is calculated as

$$
\Delta P=\frac{f_{p} L_{r e g e n} \rho_{f} v_{s f}^{2}(1-\varepsilon)}{\varepsilon^{3} D_{p}}
$$

where $L_{\text {regen }}$ is packed bed length and $f_{p}$ is friction factor. 
The viscous and inertial components are the two factors which must be added to find friction factor,

$$
f_{p 1}=\frac{150}{R e}+1.75
$$

Due to study to study variation, other coefficients are often used in practice, such as

$$
f_{p 2}=\frac{180}{R e}+1.8
$$

Going forward $f_{p 2}$ will be used, as it has been successfully used previously. Other correlations accounting for shape factor exist, but they vary widely, even within similar conditions (Ozahi et al. 2008). For these studies, sphericity is placed in the denominators of the friction factor equation,

$$
f_{p}=\frac{180}{\operatorname{Re} \theta^{2}}+\frac{1.8}{\theta}
$$

In summary, combining equations $55,56,59$ yields pressure loss,

$$
\Delta P=L_{b e d}\left(180 \frac{(1-\varepsilon)^{2}}{\theta^{2} \varepsilon^{3}} \frac{\mu_{f}}{D_{p}^{2}} v_{s f}+1.8 \frac{(1-\varepsilon)}{\theta \varepsilon^{3}} \frac{\rho_{f}}{D_{p}} v_{s f}^{2}\right)
$$

Sphericity can be used as a fit parameter in a pressure drop experiment with all other parameters known.

\subsection{2 - Pressure Drop Experiment}

The fixture for measuring pressure drop consists of a variable speed positive displacement pump, liquid feed and return lines, a regenerator, a pressure tap and transducer on both ends (Omegadyne PX309-300GI), and two in-line flow meters (GEMS 173931-C-10). 


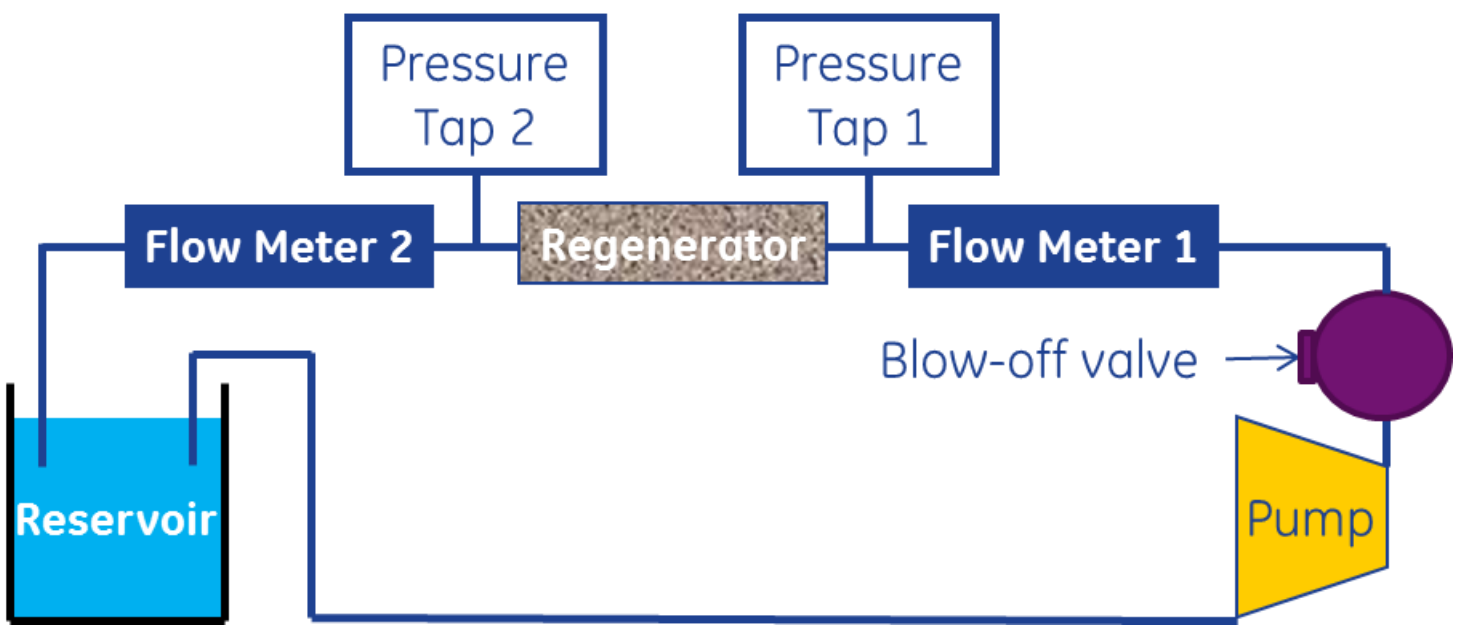

Figure 36: Diagram of pressure test apparatus

The intent is to measure steady state pressure drop across the regenerator at various flow rates. Two flow meters are used for redundancy as these meter-types are affected by any particulates in the fluid. A large discrepancy in the output of the two indicates that the system must be flushed and cleaned out. Figure 37 shows the geometry of the regenerator flow channel. This is the section through which pressure drop is being measured.

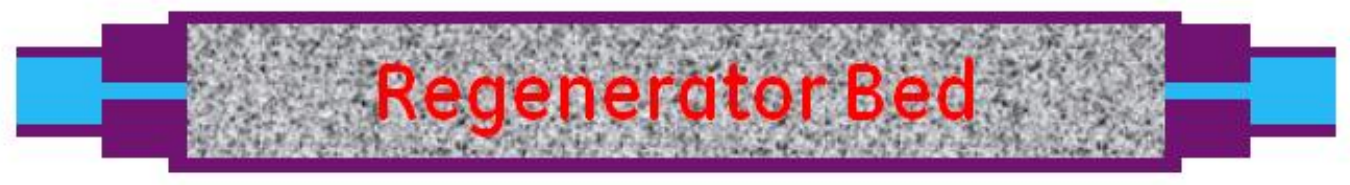

Figure 37: Regenerator cross section

Note the lack of transitional entrance length in figure 37. There are also highly restrictive flow channels at the inlet and outlet of the regenerator. Pressure drop through an empty regenerator container needs to be measured in order to separate regenerator pressure loss from inlet and outlet losses.

\subsection{3 - Critical Variables for Flow Resistance}

The main control variables are particle diameter, bed length, and particle shape. Diameter is controlled by screen sorting. Bed length is controlled directly by packing different length containers with particles. Particle sphericity is a fit parameter, to be generated from this experiment. A spherical particle baseline was performed for validation (sphericity of one). Sphericity was considered constant within each of two material families. Material family refers to the general composition and source of the magnetocaloric material. One material is chemically La-Fe-Co-Si-H, while the other is Mn-Fe-P-As. Void fraction was measured by weighing the particles in the container which has a known volume. Measured void fraction is 
about 0.42 for irregular particles, and about 0.36 for spheres. Water was used as the working fluid, and its properties were considered constant at room temperature for calculations.

Using these variables, a two by three test matrix was run for each material family. Test bed lengths were 1.4 in and 3.7 in, and particle diameters were 300-425, 425-600, and 1000-1400 $\mu m$. An additional two by three matrix was run to validate sphericity using spherical stainless steel shot. The three lengths used for these tests were 1.4, 3.4, and 12.5 inches, and the particle diameters were 363 and $635 \mu \mathrm{m}$.

\subsection{4 - Results of Sphericity Experiment}

First, the empty regenerator container was tested. It follows an inertial pressure drop curve that can be fit with a conventional model. The equation used is

$$
\Delta P_{\text {system }}=\frac{K_{\text {sys }} \rho v_{\max }^{2}}{2}
$$

where $K_{\text {sys }}=9$.

This loss coefficient was determined by minimizing the residual sum of squares for the empty container tests. The velocity was calculated at the minimum fitting diameter on the end of the regenerator, corresponding to the maximum velocity. By again minimizing the residual sum of squares, sphericity of each material family was determined. Sphericity of the Mn-Fe-P-As material was found to be 0.75 , while the La-Fe-Co-Si-H material sphericity is 0.4 . This is a large difference in shape, and could be influenced by be many different factors inherent to each process and/or chemical composition. 


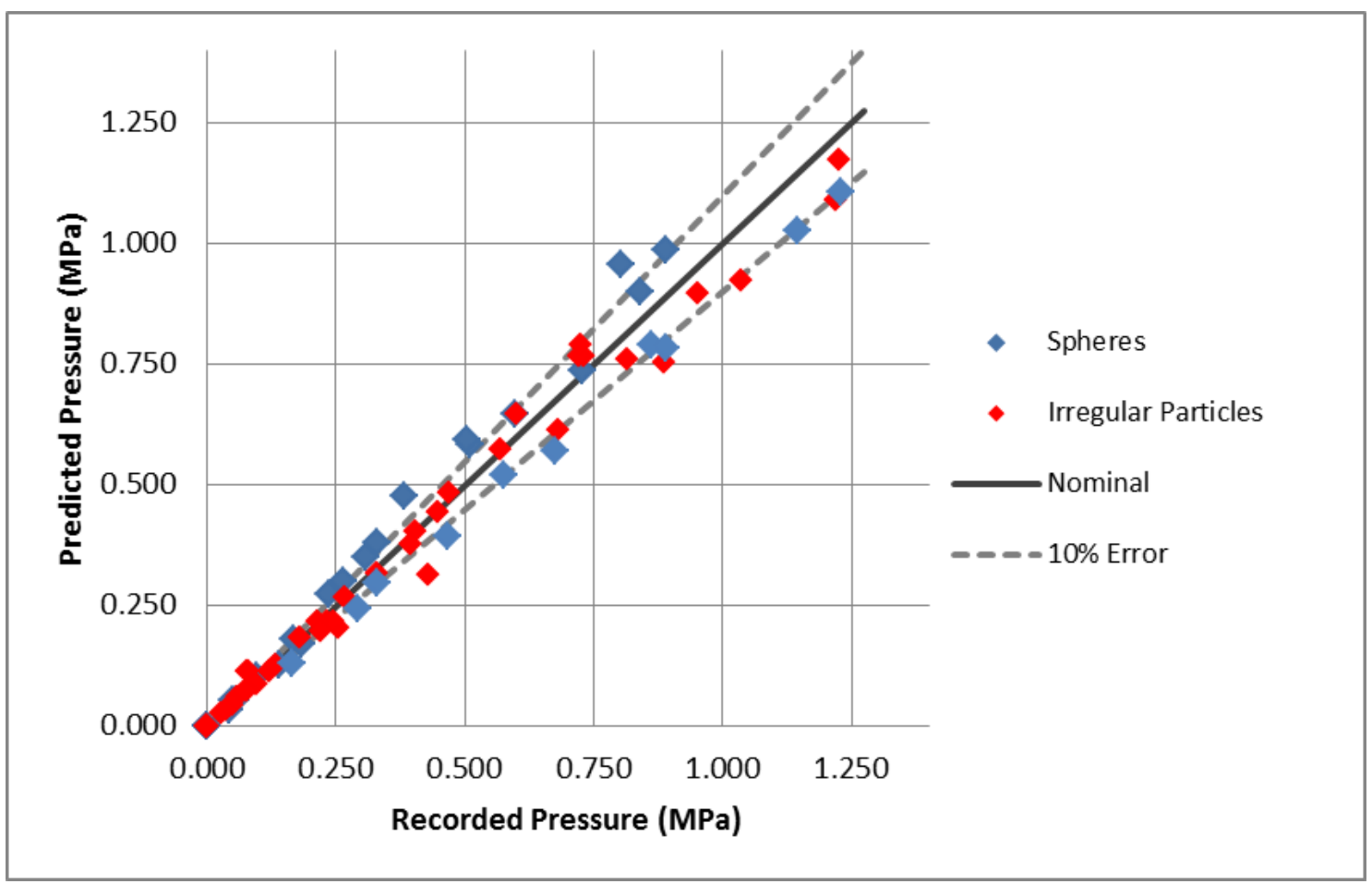

Figure 38: Regenerator pressure drop; predicted versus measured

The fit of the model is within $10 \%$ in most cases. Much of the error observed can be attributed to differences in random packing structure that cannot be avoided. Localities differ from the average properties assumed by this model. Properties such as void fraction and sphericity will vary from location to location. This amount of variation is typical for such a study on packed particle beds. 


\section{2 - Test Fixture Used for Heat Transfer Experiment}

\subsection{1 - Fixture Description}

Knowing particle sphericity, the next objective is to experimentally measure heat transfer rate. An existing test fixture was used to make said measurement and is depicted in figure 39.

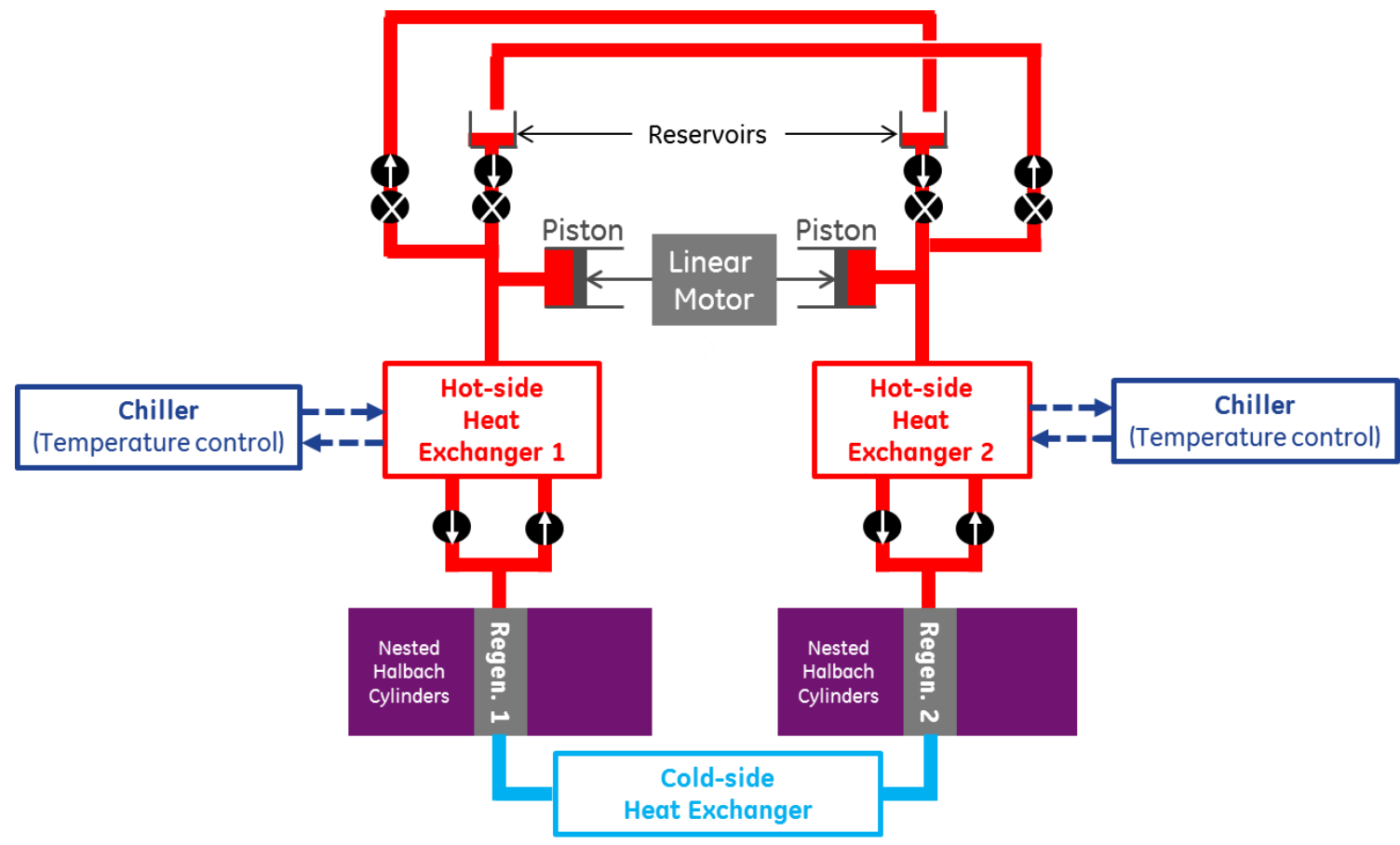

Figure 39: Magnetocaloric test fixture schematic

Although not shown, pressure taps are on the outlet of each piston cylinder. In addition temperature sensors are at the inlet and outlet of all heat exchangers. These temperature sensors are fiber optic, have extremely low thermal mass, and are specified to be accurate to $0.2 \mathrm{~K}$ absolute short term.

This machine relies on one-way valves and pistons to direct fluid flow. The pistons operate 180 degrees out of phase, such that total system fluid volume remains constant throughout each stroke. When open, the on/off valves allow flow to move in a single direction without reversal for filling and purging. In normal heat pump operation the on/off valves are closed. This means that during each stroke, one regenerator is on a cold flow cycle segment and the other on a hot flow segment. It is possible to use only one regenerator at a time by leaving the other empty. Doing this reduces the power by half, but retains the same operating condition within the single regenerator.

The hot-side temperature is controlled via a chiller. The hot-side heat exchanger is a counter flow concentric tube heat exchanger, of sufficient length to approach $100 \%$ effectiveness. A Halbach cylinder is used to magnetize and demagnetize the MCM. It is an array of magnets around the perimeter of a circle, whose cumulative field is uniform and one-directional through 
the center. The field in the nested cylinders is changed by rotating the outer cylinder, as is shown in figure 40 .
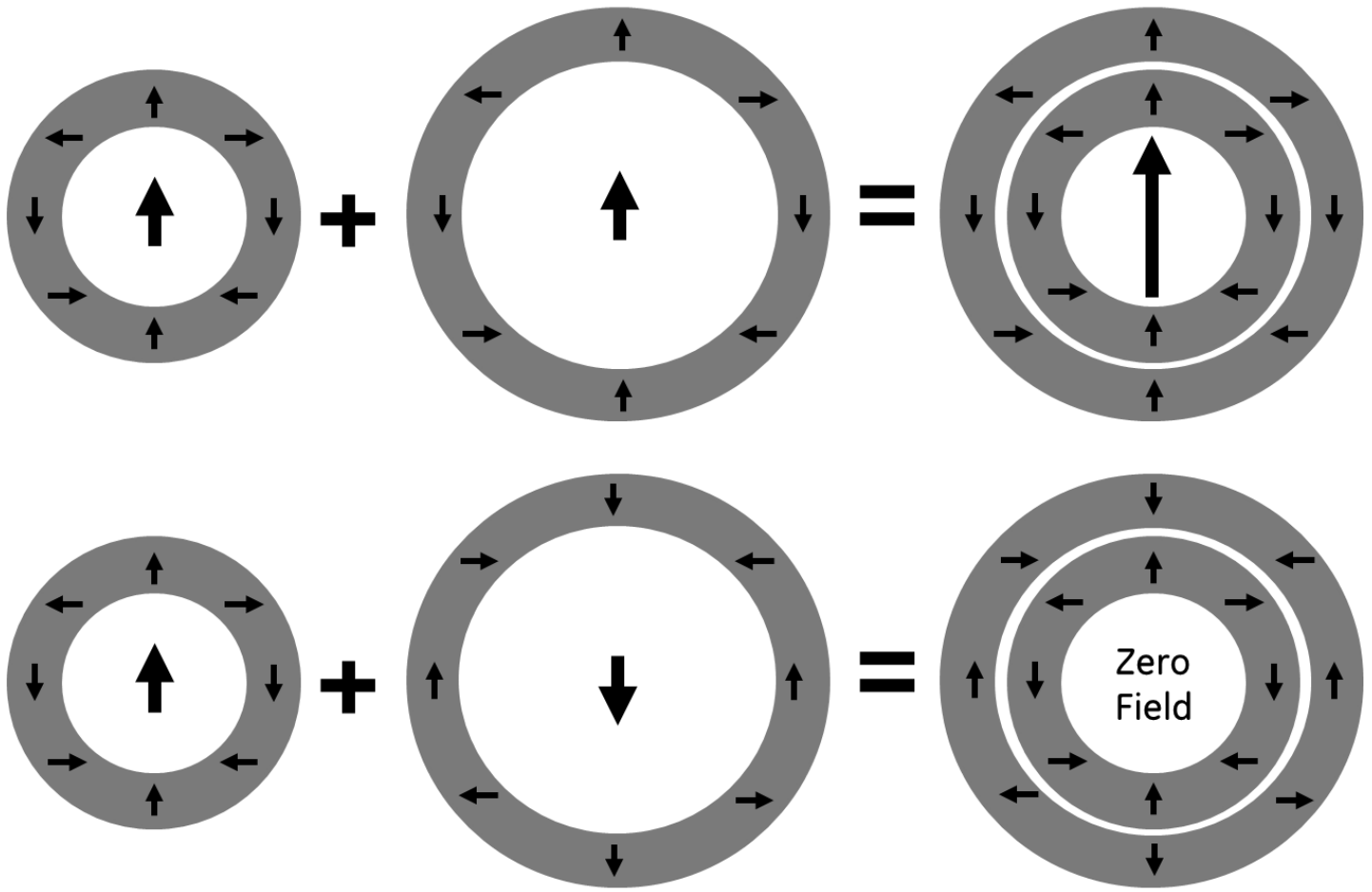

Figure 40: Nested Halbach arrays

The maximum field this system can achieve is 1.5 Tesla, while the minimum is zero. This brings up two major limitations of this device, which are magnetization intensity and time. The intensity is limited by the magnets and geometry of the arrays, while the transition time is limited by motor torque and array inertia.

\subsection{2 - Fixture Limitations}

In order to fully magnetize the material, the outer cylinder must be accelerated and stopped at 180 degrees. The minimum time to accomplish this task is determined by rotational inertia of the magnetic array and motor torque. The motor which drives both magnetic arrays can change position by 180 degrees in about $0.2 \mathrm{~s}$ at constant acceleration. By connecting the same motor to a single array, this time is cut down to $0.1 \mathrm{~s}$. This represents the fastest possible magnetization time for this machine, and is on the same order of magnitude as the expected time constants for heat transfer. This means that most of the temperature profile measurements will be made on the latter half of the transition to ambient.

Measurements cannot be made accurately while the field is changing, mainly because it introduces many more noise variables. These include accurate portrayals of magnetic array position versus time, magnetic field strength versus array position, temperature response of 
MCM versus field strength. This qualitatively means that measurements cannot be begun until the field is at full strength.

Another potential limitation is the hydraulic system. The motor which drives the hydraulic pistons is able to accelerate the pistons to over 4 inches per second in about $0.05 \mathrm{~s}$. This is double the velocity used in this experimentation. Flexibility in the system means that this displacement is not immediately translated into flow through the regenerator. Regenerator flow reaches steady state approximately 0.1 seconds after the piston begins moving. This will induce error in the initial portion of the flow period due to accelerating flow.

Both the magnetic transition time and transient flow time create a lower limit on measureable time scale, but a limitation on maximum time scale also exists. Heat leak and thermal storage of the system will skew results if a measurement takes place over a long enough time. The steady state heat leak from the regenerator has been measured to be about $0.2 \mathrm{~W} / \mathrm{K}$, which is negligible in the context of a short-term transient experiment. However, the thermal mass of the system is neglected in a steady state condition. This thermal mass is very important to a short term transient experiment as it will dilute the measured change in temperature. In order to reduce this effect, all thermal mass in contact with the working fluid and regenerator is plastic, with low conductivity and heat capacity. The correction for this effect is discussed in detail in the Heat Transfer Experiment chapter.

\section{3 - Experiment to Determine Heat Transfer Rate during Dwell}

\subsection{1 - Heat Transfer Design of Experiments}

Recall that the purpose of the experiment is to measure heat transfer rate during a dwell period, after magnetization. Both thermal transport and thermal storage related material properties are critical to a transient heat transfer problem such as this. Heat capacity and thermal conductivity must be well known for each case. In addition, certain geometric properties need to be quantified accurately. From the analytical model, particle diameter, shape, and void fraction are the key geometric variables. Of these, particle diameter is the only one that is easily controllable, via sieve sorting. Void fraction and shape can be measured, but not readily controlled.

There are two magnetocaloric materials available for testing, La-Fe-Co-Si-H and Mn-Fe-P-As. The La-Fe-Co-Si-H material has a thermal conductivity of about $8 \mathrm{~W} / \mathrm{mK}$ (Legait et al., 2014; Liu et al., 2012), while the Mn-Fe-P-As material thermal conductivity is about $2.5 \mathrm{~W} / \mathrm{mK}$ (Fujieda, 2004). Thermal heat capacities also differ slightly, but these will be considered constant and identical. Heat capacity is largely temperature dependent and was integrally averaged across the test temperature span to obtain the constant value of $900 \mathrm{~J} / \mathrm{kgK}$. Material densities are $7150 \mathrm{~kg} / \mathrm{m}^{3}$ (Legait et al., 2014) and $6200 \mathrm{~kg} / \mathrm{m}^{3}$ (Brück et al., 2013) for La-Fe-Co-Si-H and MnFe-P-As respectively. 
In order to vary working fluid properties, deionized water, ethylene glycol, and a 50-50 mix was used for a total of three different working fluids. This effectively changes fluid density, thermal conductivity, and heat capacity simultaneously. Density of the working fluid ranges from 981 to $1110 \mathrm{~kg} / \mathrm{m}^{3}$, thermal conductivity ranges from 0.58 to $0.26 \mathrm{~W} / \mathrm{mK}$, and heat capacity ranges from 4200 to $2200 \mathrm{~J} / \mathrm{kgK}$.

\begin{tabular}{|c|c|c|c|}
\hline & $\begin{array}{l}\text { Density } \\
\mathrm{kg} / \mathrm{m}^{\wedge} 3\end{array}$ & $\begin{array}{c}\text { Thermal } \\
\text { conductivity } \\
\mathrm{W} / \mathrm{mK}\end{array}$ & $\begin{array}{c}\text { Heat } \\
\text { capacity } \\
\text { J/kgK }\end{array}$ \\
\hline Water & 981 & 0.58 & 4200 \\
\hline $50-50$ & 1045 & 0.42 & 3339 \\
\hline thylene Glycol & 1110 & 0.26 & 2200 \\
\hline
\end{tabular}

Figure 41: Tabulated working fluid properties

The La-Fe-Co-Si-H material has a measured sphericity of 0.4 , while the $\mathrm{Mn}-\mathrm{Fe}-\mathrm{P}-\mathrm{As}$ has a sphericity of 0.75 . Qualitatively this means that sphericity and MCM thermal conductivity are confounded in the results. Particles sizes available range from 300 to $1000 \mu \mathrm{m}$, but the smallest tested size was determined by the measurement system limitations. The smallest particles exchange heat too rapidly to be measured by the fixture. In these cases the temperatures of the fluid and solid have approached equilibrium during the finite magnetization time. This limit in measurability corresponds to a limit in practicality of a dwell period. With such rapid heat transfer occurring, fluid temperature can be approximated to be the same as material temperature in a model with little error.

With all of this considered, the final test matrix contains two magnetocaloric materials, three different working fluids, and a maximum of three particle diameters for each case. This is a total 18 test cases at maximum. 


\begin{tabular}{|c|c|c|c|c|c|c|c|c|c|}
\hline \multirow[b]{2}{*}{ Run } & \multicolumn{6}{|c|}{ Magnetocaloric Material (MCM) } & \multicolumn{3}{|c|}{ Working Fluid } \\
\hline & Material & \begin{tabular}{|c|} 
Mean particle \\
diameter \\
$\mathrm{m}$ \\
\end{tabular} & Sphericity & $\begin{array}{l}\text { Density } \\
\mathrm{kg} / \mathrm{m}^{\wedge} 3\end{array}$ & $\begin{array}{c}\begin{array}{c}\text { Thermal } \\
\text { conductiv } \\
\mathrm{W} / \mathrm{mK}\end{array} \\
\end{array}$ & $\begin{array}{c}\text { Heat } \\
\text { capacity } \\
\text { J/kgK }\end{array}$ & $\begin{array}{l}\text { Density } \\
\mathrm{kg} / \mathrm{m}^{\wedge} 3\end{array}$ & \begin{tabular}{|c|}
$\begin{array}{c}\text { Thermal } \\
\text { conductiv } \\
\mathrm{W} / \mathrm{mK}\end{array}$ \\
\end{tabular} & $\begin{array}{c}\text { Heat } \\
\text { capacity } \\
\text { J/kgK }\end{array}$ \\
\hline 1 & $\mathrm{Mn}-\mathrm{Fe}-\mathrm{P}-\mathrm{As}$ & 0.0009 & 0.75 & 6300 & 2.5 & 900 & 981 & 0.58 & 4200 \\
\hline 2 & $\mathrm{Mn}-\mathrm{Fe}-\mathrm{P}-\mathrm{As}$ & 0.0009 & 0.75 & 6300 & 2.5 & 900 & 1045 & 0.42 & 3339 \\
\hline 3 & $\mathrm{Mn}-\mathrm{Fe}-\mathrm{P}-\mathrm{As}$ & 0.0009 & 0.75 & 6300 & 2.5 & 900 & 1110 & 0.26 & 2200 \\
\hline 4 & $\mathrm{Mn}-\mathrm{Fe}-\mathrm{P}-\mathrm{As}$ & 0.0007 & 0.75 & 6300 & 2.5 & 900 & 981 & 0.58 & 4200 \\
\hline 5 & $\mathrm{Mn}-\mathrm{Fe}-\mathrm{P}-\mathrm{As}$ & 0.0007 & 0.75 & 6300 & 2.5 & 900 & 1045 & 0.42 & 3339 \\
\hline 6 & Mn-Fe-P-As & 0.0007 & 0.75 & 6300 & 2.5 & 900 & 1110 & 0.26 & 2200 \\
\hline 7 & $\mathrm{Mn}-\mathrm{Fe}-\mathrm{P}-\mathrm{As}$ & 0.0005 & 0.75 & 6300 & 2.5 & 900 & 981 & 0.58 & 4200 \\
\hline 8 & $\mathrm{Mn}-\mathrm{Fe}-\mathrm{P}-\mathrm{As}$ & 0.0005 & 0.75 & 6300 & 2.5 & 900 & 1045 & 0.42 & 3339 \\
\hline 9 & $\mathrm{Mn}-\mathrm{Fe}-\mathrm{P}-\mathrm{As}$ & 0.0005 & 0.75 & 6300 & 2.5 & 900 & 1110 & 0.26 & 2200 \\
\hline 10 & La-Fe-Co-Si-H & 0.0011 & 0.4 & 7150 & 8 & 900 & 981 & 0.58 & 4200 \\
\hline 11 & La-Fe-Co-Si-H & 0.0011 & 0.4 & 7150 & 8 & 900 & 1045 & 0.42 & 3339 \\
\hline 12 & La-Fe-Co-Si-H & 0.0011 & 0.4 & 7150 & 8 & 900 & 1110 & 0.26 & 2200 \\
\hline 13 & La-Fe-Co-Si-H & 0.0008 & 0.4 & 7150 & 8 & 900 & 981 & 0.58 & 4200 \\
\hline 14 & La-Fe-Co-Si-H & 0.0008 & 0.4 & 7150 & 8 & 900 & 1045 & 0.42 & 3339 \\
\hline 15 & La-Fe-Co-Si-H & 0.0008 & 0.4 & 7150 & 8 & 900 & 1110 & 0.26 & 2200 \\
\hline 16 & La-Fe-Co-Si-H & 0.0005 & 0.4 & 7150 & 8 & 900 & 981 & 0.58 & 4200 \\
\hline 17 & La-Fe-Co-Si-H & 0.0005 & 0.4 & 7150 & 8 & 900 & 1045 & 0.42 & 3339 \\
\hline 18 & La-Fe-Co-Si-H & 0.0005 & 0.4 & 7150 & 8 & 900 & 1110 & 0.26 & 2200 \\
\hline
\end{tabular}

Figure 42: Tabulated design of experiments

\subsection{2 - Heat Transfer Test Procedure}

Ideally a temperature probe would directly measure fluid and solid temperatures within the regenerator immediately following magnetization. Many of these probes would then be averaged to get a bulk average temperature for each phase over time. This ideal case is not practical due the size of particles and interstitial voids in the regenerator. A probe cannot be guaranteed to touch only fluid or only solid. Even if this were the case, the probe itself will have some amount of thermal mass, which induces error in this transient case.

The real output of interest is the outgoing fluid temperature and how it is affected by a dwell period. The temperature of fluid that has exited the regenerator can be measured to determine the heat transfer rate during a dwell. The experimental procedure is shown in figure 43. 


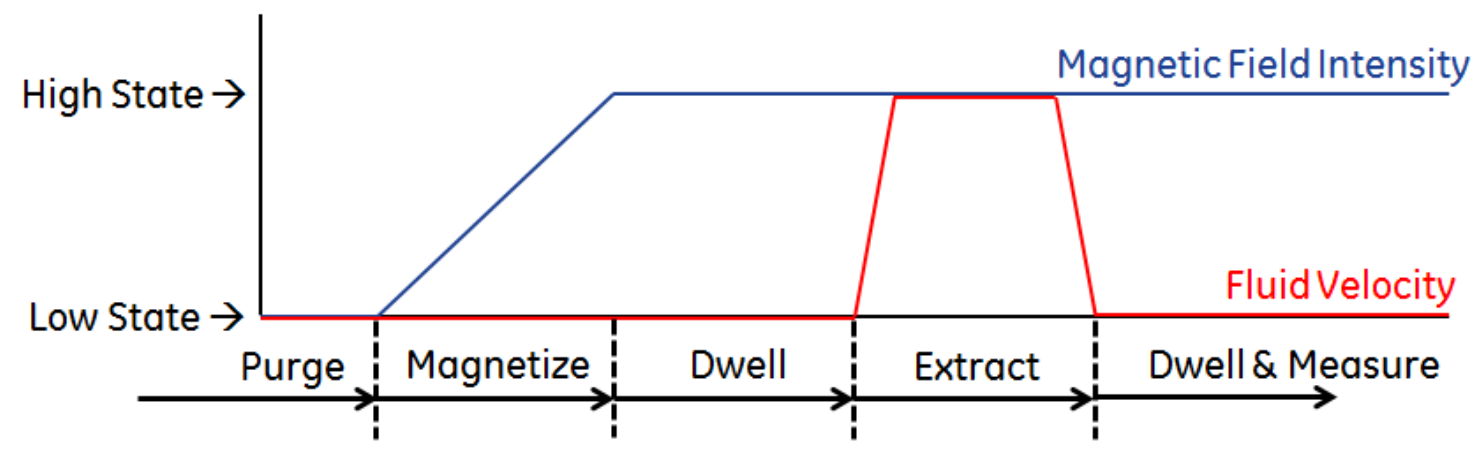

Figure 43: Experimental procedure state diagram

As shown in figure 43 the experiment starts with an extended purge period. Lasting for several minutes, this allows the MCM, working fluid, and surroundings to converge to a uniform temperature prior to the test. This is important for repeatability, as the thermal mass in contact with the system will always start at the same temperature. After the purge period the magnetic field is applied, which takes approximately $0.1 \mathrm{~s}$. At this point the MCM rises to a higher temperature, and the fluid begins receiving heat. The fluid then dwells for a period of time before being extracted from the regenerator. The fluid extracted from the very end of the regenerator is moved a short distance to a temperature probe, which records the temperature. 

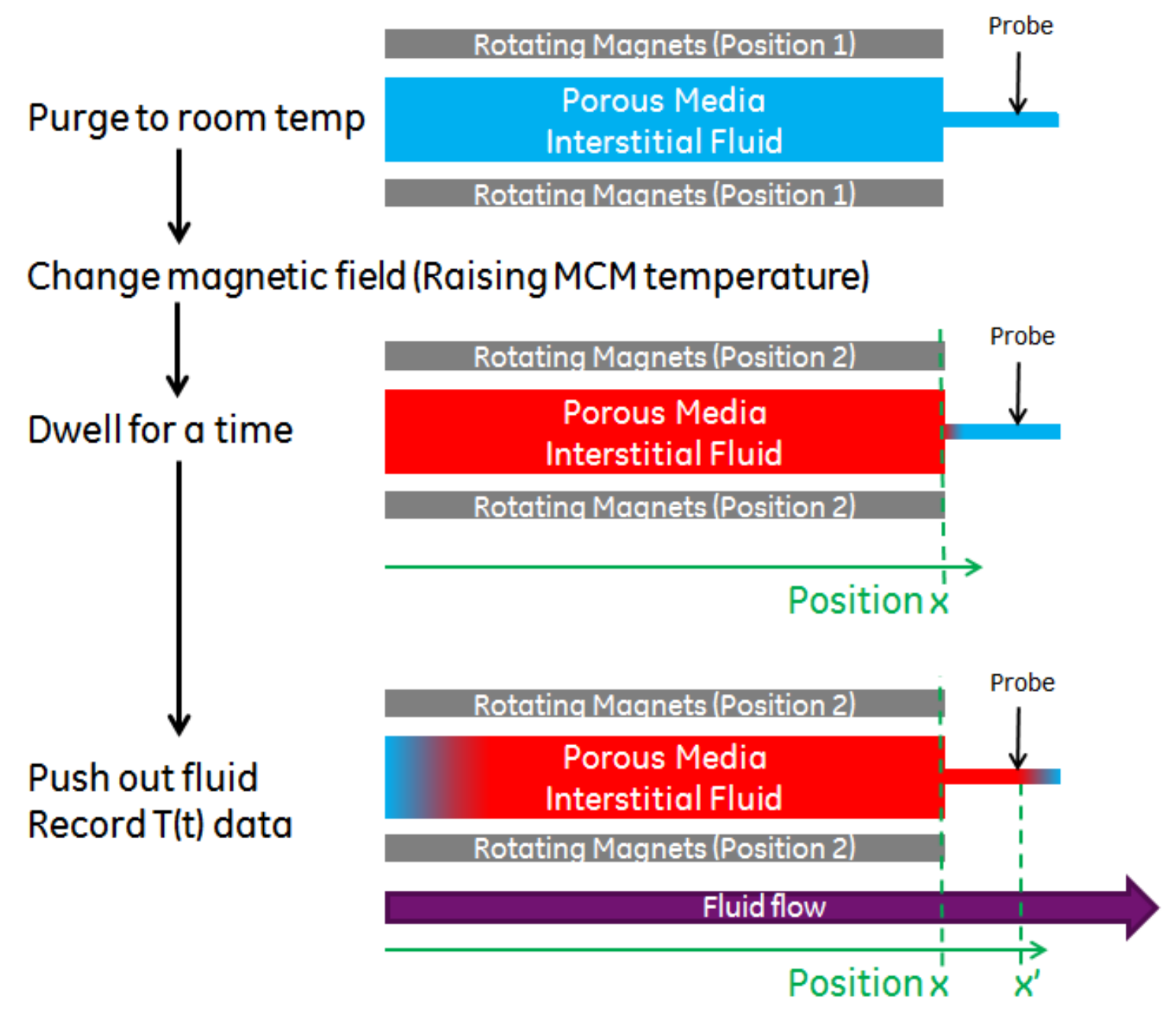

Figure 44: Regenerator during experiment

The reason for measuring the first fluid to exit the regenerator is that it does not have added contact time with the solid phase during the extraction portion of the experiment (forced convection). Any heat received by the fluid during the flow period skews results unless it's properly accounted for. This procedure needs to be repeated for several different dwell times for each case. After correcting for the thermal mass of the measurement system (tubing and sensor), the temperature versus time points can be used to back out a heat transfer time constant. This time constant can in turn yield a heat conductance (U) value. 


\subsection{3 - Processing Raw Data to Yield Heat Transfer Coefficient}

Typical fiber optic temperature sensor output for a test is shown in figure 45.

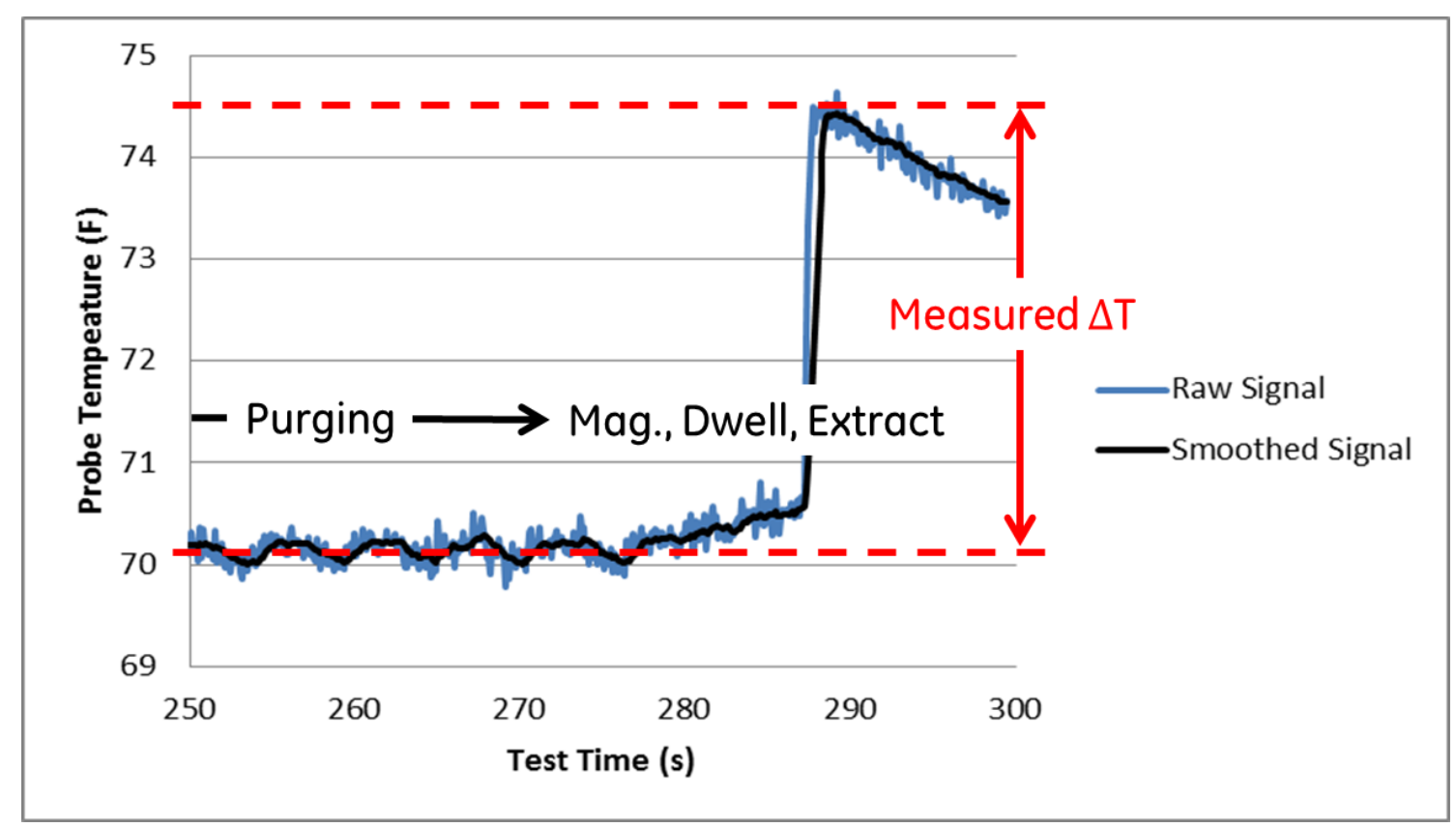

Figure 45: Raw and smoothed signal

The smoothing shown is a sliding averaging window of $1 \mathrm{~s}$, or 10 samples. More rapid sampling is not required to capture this data, as the peak is held for much longer than $0.1 \mathrm{~s}$. During the extraction period the temperature of the sensor rises, and during the measurement period the signal peaks and then begins dropping. This drop is associated with the dispersion of heat to the thermal mass surrounding the temperature sensor and fluid. Recall that all the thermal mass surrounding the sensing probe started at ambient temperature. The mass is absorbing energy from the fluid, which is at an elevated temperature leaving the regenerator. The volume of fluid being measured is extremely small, so the sensing system has enough thermal mass to influence the temperature reading transiently. The correction for this involves taking extra measurements designated for measuring the sensing system, shown in figure 46.

In reality this test is not measuring the fluid temperature. It is measuring fluid temperature after it has lost heat to the thermal mass of the measurement system. The measured temperature change is shown for a series of extraction strokes in figure 46. These all are measurements of a fluid which has reached equilibrium temperature prior to extraction. This means the fluid leaving the regenerator is at a constant temperature. 


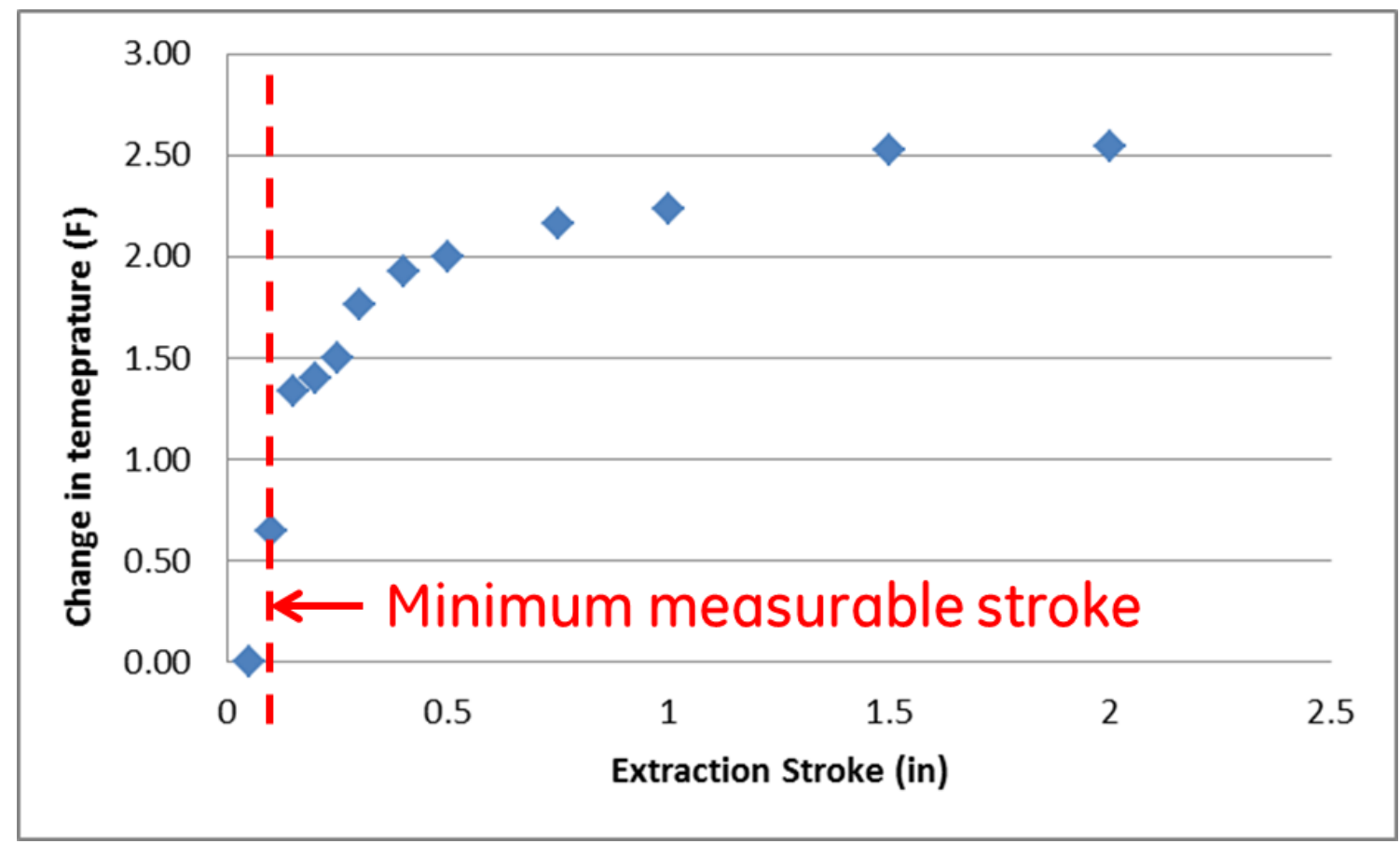

Figure 46: Measurement system thermal response

The relationship is approximately exponential, and varies with extraction velocity and working fluid composition. Convection coefficient between this outgoing fluid and the thermal mass of the system is constant because fluid velocity is constant; meaning that the only variable is time. This particular case was performed with pure water, using a stroke velocity of $2 \frac{\mathrm{in}}{\mathrm{s}}$. As the stroke increases, so does the measured change in temperature. Longer strokes at constant velocity provide more and more contact time, bringing the observed temperature closer and closer to true outgoing fluid temperature. If the full fluid volume of the regenerator is exceeded in an extraction stroke the profile will change. This case was avoided in all tests. The temperature change observed with a two inch stroke is effectively the true fluid temperature change for the case when the fluid is in thermal equilibrium with the MCM. This also illustrates the stroke required to capture the first fluid to exit the regenerator.

The time constant of this system response can be used as a transfer function, to translate a measured temperature change at minimum extraction stroke to an actual fluid temperature leaving the regenerator. A total of three points must be run for each test, as is shown in figure 47. 


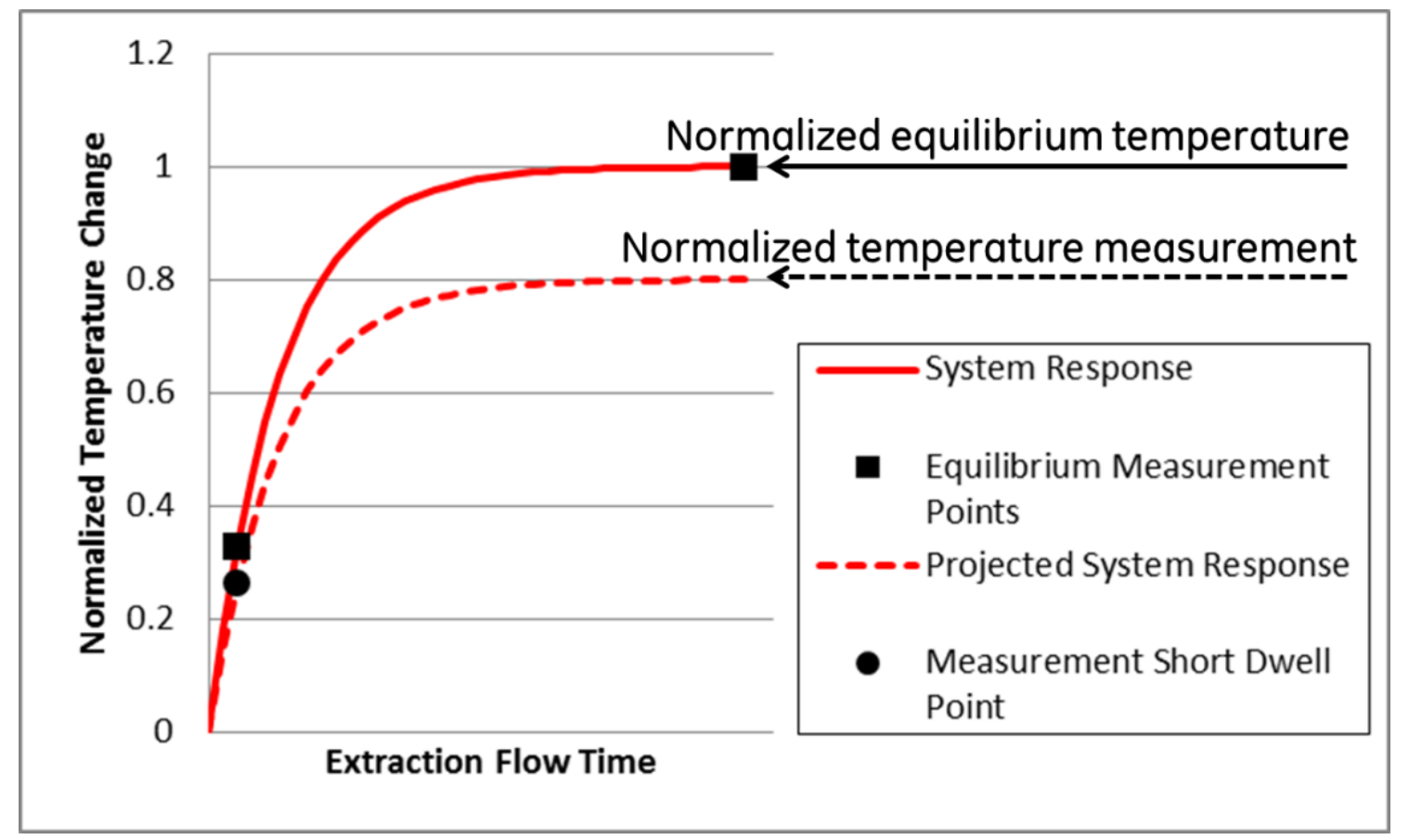

Figure 47: Finding fluid outlet temperature using system response

In the case shown in figure 47 , the dwell time utilized allowed the fluid to achieve $80 \%$ of possible temperature change. In order to find this value, three test points were determined. First, the minimum stroke length and long stroke length points were used to determine the measurement system time constant under the current conditions,

$$
\tau_{\text {c sys }}=-\frac{t_{\text {min }}}{\operatorname{ltroke}}
$$

where $\tau_{c \text { sys }}$ is measurement system time constant for the specific fluid type and flow rate, $t_{\text {stroke }}^{\min }$ is time required to move the minimum measureable stroke distance, $\Delta T_{\infty \mathrm{min}}$ is measured temperature change using a long dwell period and the minimum stroke

stroke, and $\Delta T_{\text {stroke }}^{\infty \max }$ is measured temperature change using a long dwell period and the longest stroke.

Next, the actual test point is run and normalized to the equilibrium long stroke point. The previously determined system time constant is applied to the measurement to calculate actual fluid temperature,

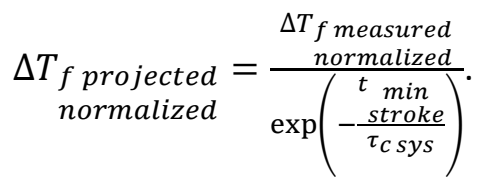


where $\Delta T_{f \text { projected }}$ is normalized fluid outlet temperature, projected from the normalized

measured point and $\Delta T_{f}$ measured is normalized fluid measured temperature. normalized

The reason for normalizing the temperature response is to maintain comparability between tests, regardless of material temperature response. Recall that this response is highly dependent on absolute temperature and magnetic field strength. This method of using normalized temperature change effectively eliminates ambient temperature as a noise variable, so long as temperature fluctuations occur slowly over time. Any drift in room temperature over time should not affect results.

This method of measurement also requires the approximation of constant outlet temperature. The system response is being measured with a constant temperature input. Because the measured fluid is the first fluid to leave the regenerator, a near constant temperature should hold true. If a measurement where taken of fluid deeper inside the regenerator, this would no longer hold true.

Running the test for several different dwell times yields a profile of points, as is shown in figure 48.

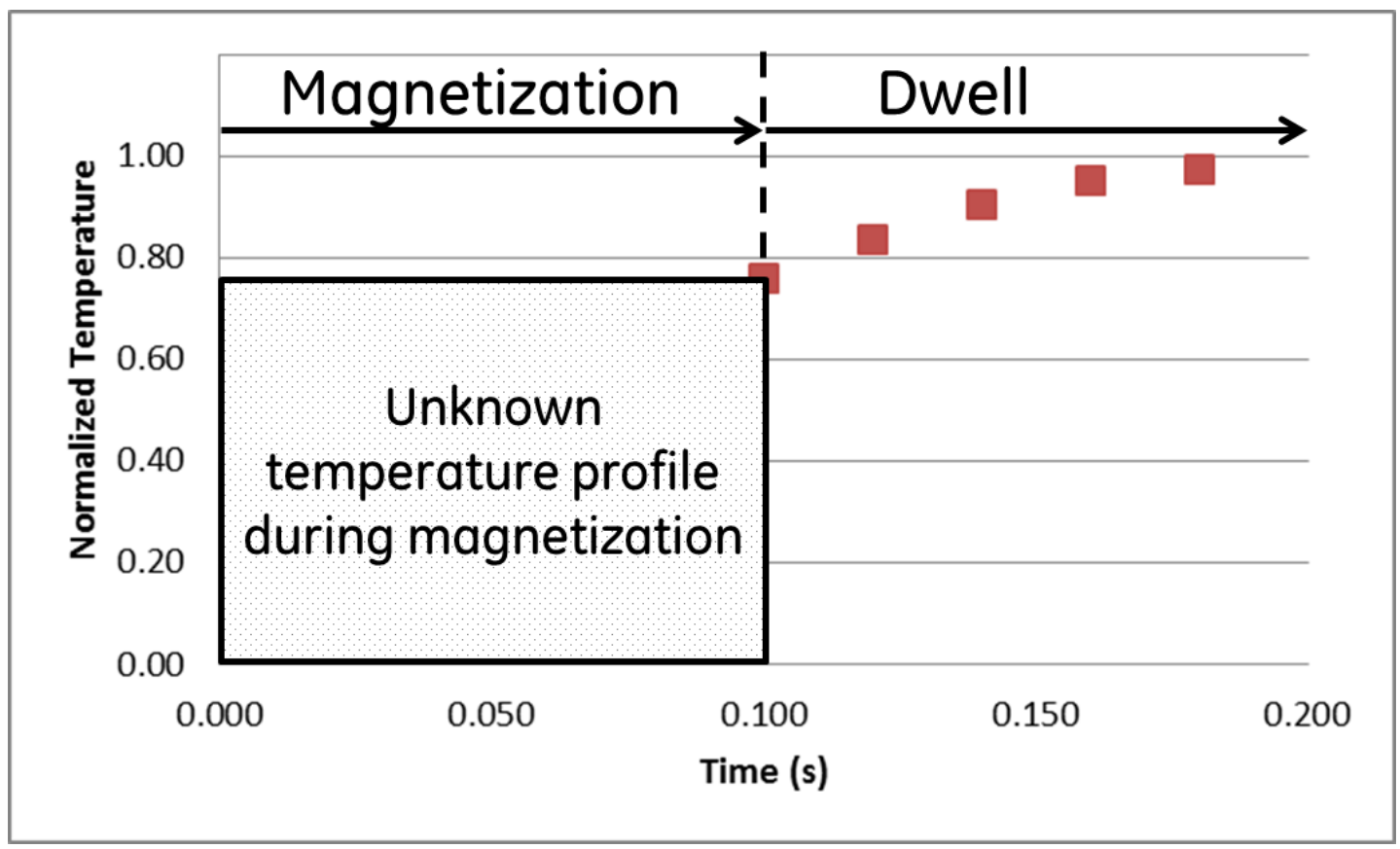

Figure 48: Normalized fluid outlet temperature vs. test time

The time scale on this set of results includes magnetization time. For this particular case $75 \%$ of heat was transferred during magnetization. The remainder of heat was transferred within the 
following $0.1 \mathrm{~s}$. To account for run to run variability, multiple sets of points were run and averaged for each test case.

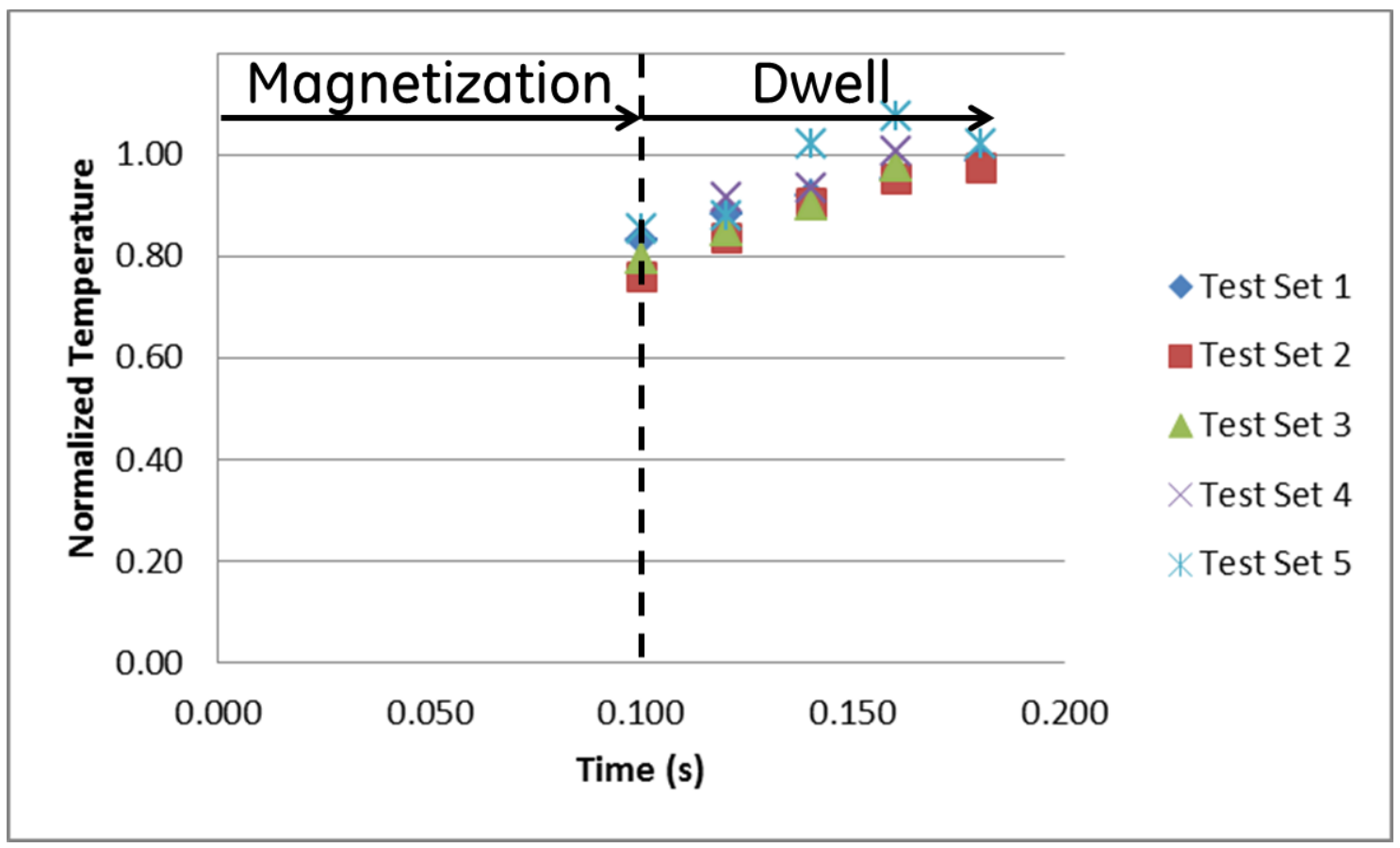

Figure 49: Combining multiple profile sets

The points for each particular dwell time in figure 49 were averaged, and an exponential curve was fitted to the points. Recall the assumption made in the analytical section, that a single exponential function can be used to model the temperature profile. Once the form of the equation is assumed with normalized output $(0-1)$, only two unknown parameters remain. These are time constant and starting time. This can be reduced to a single term, time constant, by using a floating starting point on the time axis, which allows the fit to be independent of heat transferred during the magnetization period. The only remaining fit parameter is observed time constant.

The final step is to translate the observed time constant into a $U$ value. Recall that

$$
\tau_{c \text { coupled }}=\frac{1}{U A_{h x}} \frac{\varepsilon\left(\rho C_{p}\right)_{f}(1-\varepsilon)\left(\rho C_{p}\right)_{s}}{\left(\varepsilon\left(\rho C_{p}\right)_{f}+(1-\varepsilon)\left(\rho C_{p}\right)_{s}\right)} .
$$

where $\tau_{c \text { coupled }}$ is observed time constant.

Using a measured (fitted) time constant, heat transfer coefficient for each test case is

$$
U=\frac{1}{\tau_{c \text { coupled }} A_{h x}} \frac{\varepsilon\left(\rho C_{p}\right)_{f}(1-\varepsilon)\left(\rho C_{p}\right)_{s}}{\left(\varepsilon\left(\rho C_{p}\right)_{f}+(1-\varepsilon)\left(\rho C_{p}\right)_{s}\right)}
$$


where

$$
A_{h x}=\frac{6(1-\varepsilon)}{D_{p} \theta} .
$$




\section{5 - HEAT TRANSFER RESULTS}

\section{1 - Comparison between Measured and Predicted UA Values}

Each calculated $U$ value comes from at least five temperature profiles, with at least five points each. In other words, all of the points shown in figure 49 produce a single $U$ value. These points amount to more than 25 dwell measurements per $U$ value. Of the possible 18 test cases, only nine were measurable using the equipment. The rest of the material transferred heat too quickly to get an accurate measurement using this method. This means that the resulting correlation is the result of just over 300 dwell measurements in total. The average measured UA values per unit regenerator volume are plotted for each of the nine test cases in figure 50 .

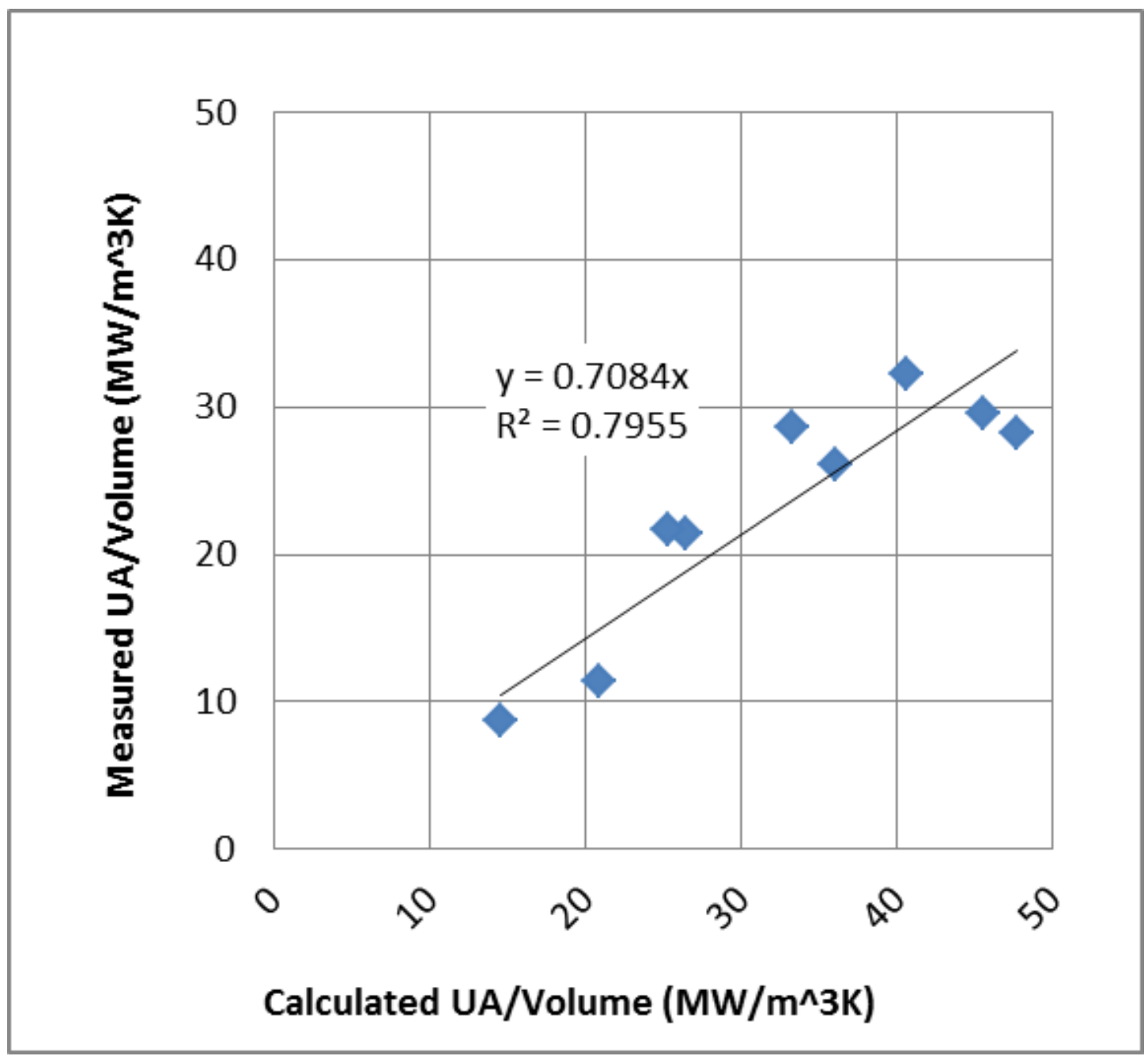

Figure 50: Average measured versus calculated specific UA values 
The R-squared value for the correlation is about 0.8 , suggesting that the model is capturing the critical variables and effects. However, the slope of the fit curve shows that the measured values are $30 \%$ lower than the calculated values using the model presented. This could be due to any number of noise variables. By applying a multiplied correction factor of 0.70 to the output of the analytical model, maximum model error becomes $20 \%$. This makes the final correlation for heat transfer coefficient

$$
U=\frac{0.70}{\left(\frac{L_{C S}}{k_{S}}+\frac{L_{c f}}{k_{f}}\right)}=\frac{0.70}{\left(\frac{D_{p} \theta}{6 k_{S}}+\frac{D_{p \theta} \varepsilon}{6(1-\varepsilon) k_{f}}\right)} .
$$

where $\theta$ is sphericity ( 0 to 1 ), $D_{p}$ is mean particle diameter (Using sieve hole sizes used in sorting), $\varepsilon$ is void fraction in regenerator packing, $k_{s}$ is solid material conductivity, and $k_{f}$ is fluid material conductivity.

As a point of reference, the equivalent fluid characteristic length using typical void fraction and spherical particles is

$$
L_{c f}=\frac{D_{p \theta}}{6} \frac{\varepsilon}{(1-\varepsilon)} \frac{1}{0.70}=\frac{D_{p}}{5.8}
$$

\section{2 - Creation of a Continuous Function Using a Flow Correlation}

The conductive heat transfer coefficient can be superimposed with flow correlations in order to create a continuous heat transfer rate function. Recall, the Wakao correlation,

$$
N u=2+1.1 \operatorname{Re}^{0.6} \operatorname{Pr}^{1 / 3}
$$

Using the definition of Nusselt number,

$$
N u=\frac{h D}{k}
$$

and the fluid portion of the newly acquired total $U$ value (equation 65 ),

$$
\underset{\text { only }}{U_{\text {fluid }}}=h=\frac{0.70}{\left(\frac{L_{c f}}{k_{f}}\right)}=\frac{0.70}{\left(\frac{D_{p \theta} \varepsilon}{6(1-\varepsilon) k_{f}}\right)},
$$

a new correlation constant can be determined for equation 9. This yields a modified Wakao correlation, of the form 


$$
N u=0.70 \frac{6(1-\varepsilon)}{\theta \varepsilon}+1.1 \operatorname{Re}^{0.6} \operatorname{Pr}^{1 / 3}
$$

In the context of this experiment, internal resistance of the solid material cannot be neglected. Therefore, the resulting convection coefficient $(h)$ from this correlation must be taken in series with the solid conduction component:

$$
U=\frac{1}{\frac{1}{h}+\frac{L_{C S}}{0.70 k_{S}}}
$$

\section{3 - Discussion on Variability within the Experiment}

The amount of variability observed in figure 50 is not uncommon for packed particle bed experimentation, as many local differences exist within a random packing of particles. Beyond geometric variability, magnetocaloric material properties vary dramatically within the temperature span of the test. An average $C p$ value was used in the thermal mass calculations, which will certainly induce some amount of error into the model. Other material properties, such as thermal conductivity, may vary due to the method of measurement. The thermal conductivity used for the MCM in the model was measured as a bulk average. When the material is granulated to near the order of size of a crystallite, bulk properties may no longer be valid. Grain boundaries and can create directional and local difference in properties such as thermal conductivity.

Another consideration regarding error is that this is really a measurement of the fluid exiting the regenerator upon starting flow. Although this is very representative of real machine performance, it may not be exactly represented by the analytical model. The analytical model is for the fluid as it dwells in the interstitial voids of the packed bed. Between the dwell and the measurement is a flow period, which expels and mixes the fluid to an average outlet temperature. The assumption is that when fluid is displaced to be read by the sensor, all fluid travels at the same axial velocity and is mixed prior to reaching the sensor. This means that the measured temperature is a true bulk average of the cross section of fluid. It's possible that the expelled fluid is mainly from the larger voids, where there is less resistance to flow locally. This fluid would also be the last to reach equilibrium, as the conduction distance is largest. Qualitatively, this effect would reduce the observed heat transfer coefficient, just as was observed.

One more effect that could pull the observed heat transfer rate down is corrosion. The magnetocaloric material has been observed to change color slightly over time, suggesting a reaction with the working fluid. Corrosion inhibitor was added to the working fluid, but corrosion effects cannot be ruled out completely without test repetition at regular time 
intervals. An oxide layer on the exterior of the particles would increase thermal resistance between the solid and liquid, effectively slowing heat transfer.

The same outcome would be apparent with erosion as corrosion. Void fraction could be increased over time by particles becoming more rounded. This would increase average conduction distance, again decreasing the observed heat transfer rate. If pressure drop data were taken over time this could be proven true or false. A falling pressure drop at constant flow rate would suggest erosion. 


\section{6 - SUMMARY OF FINDINGS}

In summary, the addition of a dwell period to the typical magnetocaloric cycle was shown to have a positive performance impact in specific conditions. These conditions correspond to regenerators that are heat transfer rate limited in operation. This allows regenerators to be used that offer lower flow resistance, potentially improving machine efficiency.

Next, the heat transfer between the solid and fluid phases of a packed bed active regenerator was explored. The heat transfer mechanism was shown to be pure conduction via a CFD study. Toroidal convective currents were present, but flow velocity was miniscule. Assuming pure conduction, a model was created to calculate average heat transfer coefficient from geometric parameters and material properties. This model relies on a generic characteristic length calculation for both the fluid and solid phases within the regenerator. This heat transfer coefficient was rolled into a coupled two mass exponential model, which was validated via a time stepping model. The coupled exponential model dramatically decreases the number of calculations required to run the model.

A pressure drop experiment was done to separately measure particle sphericity. The results allowed for an independent measurement of heat transfer area. Next, an experiment was created to measure the fluid temperature over time after magnetization. Parameters such as particle diameter, particle thermal conductivity, and fluid thermal conductivity were varied to assess the modeled heat transfer coefficient. The coupled exponential model was fitted to the output of these tests, leading to a final correlated model. The analytical model required a scaling factor of 0.70 to be applied to calculate heat transfer rate, making the final correlation

$$
U=\frac{0.70}{\left(\frac{L_{c S}}{k_{S}}+\frac{L_{c f}}{k_{f}}\right)}=\frac{0.70}{\left(\frac{D_{p} \theta}{6 k_{S}}+\frac{D_{p \theta} \varepsilon}{6(1-\varepsilon) k_{f}}\right)}
$$

where $\theta$ is sphericity (0 to 1 ), $D_{p}$ is mean particle diameter (Using sieve hole sizes used in sorting), $\varepsilon$ is void fraction in regenerator packing, $k_{s}$ is solid material conductivity, and $k_{f}$ is fluid material conductivity.

Finally, if a continuous function for heat transfer rate is required for both stagnant and flowing conditions, a superimposition can be made with an existing flow correlation. In a Nusselt versus Reynolds correlation, this means changing or adding a constant. If the solid material internal resistance is not negligible, then a series thermal resistance must be subsequently calculated. 


\section{1 - Future Work}

Several opportunities for additional future have come about while completing this work. The most obvious is the addition of more variables to the test matrix. Additional magnetocaloric materials could be used and sphericity could be separated from MCM conductivity experimentally. Along these same lines, spheres could be tested in order to baseline a more well-known geometry. Gadolinium would be an excellent candidate for this baseline, as its properties have been studied quite extensively. Another technology which has gained some leverage in the field is bonded beds. These beds are held together with a very thin layer of resin, which may affect heat transfer significantly. A heat transfer measurement of the effect of bonding would be a very useful point.

The concept of heat transfer with no bulk fluid motion equally applies to other regenerator geometries. It would be beneficial for the field to have similar heat transfer correlations for micro-channels, parallel plates, and other porous media used in regenerators. Understanding the heat transfer for such shapes will allow the optimal machine to be designed.

Regarding the experiment itself, some improvements could also be made. Using electromagnets to magnetize the material would allow the magnetization time to be reduced dramatically. With this in place, a larger portion of the temperature versus time profile could be sampled. This would also allow smaller particles to be sampled than those used in the current work. 


\section{REFERENCES}

Aliev, A. M., Gamzatov, A. G., Kalitka, V. S., \& Kaul, A. R. (December 01, 2011). Low field magnetocaloric effect and heat capacity of A-site ordered NdBaMn"2O"6 manganite. Solid State Communications, 151, 23, 1820-1823.

Alonso, M., Sainz, E., Lopez, F. A., \& Shinohara, K. (January 01, 1995). Void-size probability distribution in random packings of equal-sized spheres. Chemical Engineering Science, 50, 12, 1983-1988.

Aprea, C., Greco, A., \& Maiorino, A. (October 10, 2013). A dimensionless numerical analysis for the optimization of an active magnetic regenerative refrigerant cycle. International Journal of Energy Research, 37, 12, 1475-1487.

Awuah, G. B., Ramaswamy, H. S., \& Simpson, B. K. (February 01, 1993). Surface Heat Transfer Coefficients Associated with Heating of Food Particles in CMC Solutions. Journal of Food Process Engineering, 16, 1, 39-57.

Barcza, A., Katter, M., Zellmann, V., Russek, S., Jacobs, S., \& Zimm, C. (October 01, 2011). Stability and magnetocaloric properties of sintered $\mathrm{La}(\mathrm{Fe}, \mathrm{Mn}, \mathrm{Si})<\mathrm{sub}>13</$ sub $>\mathrm{H}<\mathrm{sub}>\mathrm{z}</$ sub $>$ alloys. leee Transactions on Magnetics, 47, 10, 3391-3394.

Bouchard, J., Nesreddine, H., \& Galanis, N. (February 01, 2009). Model of a porous regenerator used for magnetic refrigeration at room temperature. International Journal of Heat \& Mass Transfer, 52.

Brück, E.H., Van Dijk, N.H., \& Ou, Z.Q. (2013). Magnetic structure and phase formation of magnetocaloric Mn-Fe-P-X compounds. (doctoralThesis.) Uitgeverij BOXPress, 's-Hertogenbosch.

Churchill, S. W. (January 01, 1977). A comprehensive correlating equation for laminar, assisting, forced and free convection. Aiche Journal, 23, 1, 10-16.

Denton, W. H., Robinson, C. H., Tibbs, R. S., \& United Kingdom Atomic Energy Authority. (1963). The heat transfer and pressure loss in fluid flow through randomly packed spheres. Harwell, Berkshire, England: Scientific Administration Office, Atomic Energy Research Establishment. Engelbrecht, K. (2005). A numerical model of an active magnetic regenerator refrigeration system. 
Ergun, S. (February 01, 1952). Determination of Geometric Surface Area of Crushed Porous Solids. Analytical Chemistry, 24, 2, 388-393.

Ergun, S., \& Orning, A. A. (June 01, 1949). Fluid Flow through Randomly Packed Columns and Fluidized Beds. Industrial \& Engineering Chemistry, 41, 6, 1179-1184.

Fujieda, S., Hasegawa, Y., Fujita, A., \& Fukamichi, K. (March 01, 2004). Thermal transport properties of magnetic refrigerants $\mathrm{La}\left(\mathrm{Fex}^{\sim} \mathrm{Si}^{\sim} \sim \sim \mathrm{X}^{\sim}\right) 1 \sim 3 \sim$ and their hydrides, and Gd5 $\sim \mathrm{Si} \sim \mathcal{G e 2} \sim$ and MnAs. Journal of Applied Physics, 95, 2429-2431.

Gao, Q., Yu, B. F., Wang, C. F., Zhang, B., Yang, D. X., \& Zhang, Y. (January 01, 2006). Experimental investigation on refrigeration performance of a reciprocating active magnetic regenerator of room temperature magnetic refrigeration. International Journal of Refrigeration, $29,8,1274-1285$.

Globe, S., and D. Dropkin (1959). Journal of Heat Transfer, 81C, 24.

Gnielinski, V., Gleichungen zur Berechnung des Wärme- und Stoffaustausches in durchströmten ruhenden Kugelschüttungen bei mittleren und grossen Pecletzahlen, Verfahrenstechnik 12(6), 63-366, 1978

Gunn, D. J. (April 01, 1978). Transfer of heat or mass to particles in fixed and fluidised beds. International Journal of Heat and Mass Transfer, 21, 4, 467-476.

Incropera, F. P. (2007). Introduction to heat transfer. Hobokenm NJ: Wiley.

Kays, W. M., \& London, A. L. (1984). Compact heat exchangers. New York: McGraw-Hill.

Kuhn, L. T., Pryds, N., Bahl, C. R. H., Smith, A., \& Joint European Magnetic Symposia, JEMS 2010. (August 19, 2011). Magnetic refrigeration at room temperature - From magnetocaloric materials to a prototype. Journal of Physics: Conference Series, 303, 1.

Kunii, D., Levenspeil, O., 1969, Fluidization Engineering, Wiley, New York, NY, 534.

Lee, J. S. (June 01, 2004). Evaluation of the magnetocaloric effect from magnetization and heat capacity data. Physica Status Solidi (b), 241, 7, 1765-1768.

Legait, U., Guillou, F., Kedous-Lebouc, A., Hardy, V., \& Almanza, M. (January 01, 2014). An experimental comparison of four magnetocaloric regenerators using three different materials. International Journal of Refrigeration, 37, 147-155.

Liu, J., Moore, J. D., Skokov, K. P., Krautz, M., Lowe, K., Barcza, A., Katter, M., ... Viewpoint Set No. 51: Magnetic Materials for Energy. (September 01, 2012). Exploring La(Fe,Si)"1"3-based magnetic refrigerants towards application. Scripta Materialia, 67, 6, 584-589. 
Nielsen, K. K., Bahl, C. R. H., Smith, A., Bjørk, R., Pryds, N., \& Hattel, J. (September 01, 2009). Detailed numerical modeling of a linear parallel-plate Active Magnetic Regenerator.

International Journal of Refrigeration, 32, 6, 1478-1486.

Ozahi, E., Gundogdu, M. Y., \& Carpinlioglu, M. O. (January 01, 2008). A Modification on Ergun's Correlation for Use in Cylindrical Packed Beds With Non-spherical Particles, Advanced Powder Technology, 19, 4, 369-381.

Pacific Northwest National Laboratory (U.S.), United States., United States., Brown, D. R., Dirks, J. A., Fernandez, N., \& Stout, T. E. (2010). The Prospects of Alternatives to Vapor Compression Technology for Space Cooling and Food Refrigeration Applications. Washington, D.C: United States. Dept. of Energy.

Pecharsky, V. K., \& Gschneidner, J. K. A. (January 01, 1999). Magnetocaloric effect and magnetic refrigeration. Journal of Magnetism and Magnetic Materials, 200, 44-56, Chapter 1, figure 1.1.

Petersen, T., Pryds, N., Smith, A., Hattel, J., Schmidt, H., \& Hogaardknudsen, H. (May 01, 2008). Two-dimensional mathematical model of a reciprocating room-temperature Active Magnetic Regenerator. International Journal of Refrigeration, 31, 3, 432-443.

Rowe, A., \& Tura, A. (January 01, 2006). Experimental investigation of a three-material layered active magnetic regenerator. International Journal of Refrigeration, 29, 8, 1286-1293.

Scott, G. D., \& Kilgour, D. M. (June 01, 1969). The density of random close packing of spheres. Journal of Physics D: Applied Physics, 2, 6, 863-866.

Wadell, H. (May 01, 1935). Volume, Shape, and Roundness of Quartz Particles. The Journal of Geology, 43, 3, 250-280.

Wakao, N., Kaguei, S., \& Funazkri, T. (January 01, 1979). Effect of fluid dispersion coefficients on particle-to-fluid heat transfer coefficients in packed beds:Correlation of nusselt numbers.

Chemical Engineering Science, 34, 3, 325-336.

Wakao, N., \& Kagei, S. (1982). Heat and mass transfer in packed beds. New York: Gordon and Breach Science Publishers.

Whitaker, S. (March 01, 1972). Forced convection heat transfer correlations for flow in pipes, past flat plates, single cylinders, single spheres, and for flow in packed beds and tube bundles. Aiche Journal, 18, 2, 361-371. Yu, B. F., Gao, Q., Zhang, B., Meng, X. Z., \& Chen, Z. (September $01,2003)$. Review on research of room temperature magnetic refrigeration. International Journal of Refrigeration, 26, 6.) 


\section{APPENDIX I - LIST OF VARIABLES}
$\alpha$ : Thermal diffusivity
K: Pressure loss coefficient
$\varepsilon$ : Void fraction
$\mathrm{k}$ : Thermal conductivity
$\mu$ : Dynamic viscosity
L: Length
0: Sphericity
$\mathrm{L}_{\mathrm{c}}$ : Characteristic length
$\rho$ : Density
m: Mass
$\tau_{c}$ : Time constant
$\mathrm{N}$ : Count, number
A: Area
$\mathrm{Nu}$ : Nusselt number
$A_{h x}$ : Heat transfer area per unit
P: Pressure
volume
Pr: Prandtl number
Bi: Biot number
COP: Coefficient of performance
Re: Reynolds number
$\mathrm{C}_{\mathrm{p}}$ : Heat capacity
S: Entropy
$\mathrm{D}_{\mathrm{p}}$ : Particle diameter (Average)
St: Stanton number
E: Energy or work
$\mathrm{T}$ : Temperature
$\dot{E}$ : Energy or work rate
$\dot{\mathrm{T}}: \frac{\mathrm{dT}}{\mathrm{dt}}, 1^{\text {st }}$ derivative of temperature
Fo: Fourier number
t: Time
$\mathrm{f}_{\mathrm{p}}$ : Friction factor
U: Heat transfer coefficient
V: Volume
H: Enthalpy
h: Convection coefficient
v: Velocity 


\section{APPENDIX II - LIST OF SUBSCRIPTS}

$\infty$ : Final, equilibrium, as $t$ approaches infinity

ad: Adiabatic

bed: Regenerator bed, packed bed

cool: Cold-side or heat absorption side

dwell: Referring to static fluid

condition

f: Fluid

flow: Referring to flowing fluid

condition

hot: Hot-side or heat rejection side

hx: Heat exchange

i: Initial, starting, at $\mathrm{t}=0$

in: Input

max: Maximum value

min: Minimum value $\mathrm{n}$ : Current node

$n+1$ : One node away in positive ' $x$ '

direction

normalized: Scaled to a value from 0

to 1

out: Output

s: Solid, MCM

segment: Referring to a node, or axial segment

sf: Superficial

step: Step size

stroke: Fluid displacement piston

stroke

sys: System

t: Current time step

$t+1$ : One time step in the future

useful: Useful, utilized 


\title{
CURRICULUM VITAE
}

\author{
NAME: $\quad$ Michael G Schroeder \\ ADDRESS: $\quad 7815$ Bridlewood Place \\ Louisville, KY 40228 \\ DOB: $\quad$ August $11,1988-$ Lima, $\mathrm{OH}$ \\ EDUCATION: B.S., Mechanical Engineering \\ University of Toledo \\ $2007-2011$
}

INDUSTRY

EXPERIENCE: Engineering co-op, General Electric Appliances - Louisville, KY

Fall, 2009 - Dishwasher energy usage modeling and optimization

Summer, 2010 - Dishwasher hydraulics design: 2 patents awarded for reversing spray arms and a novel water diverter

Spring, 2011 - Clothes washer dynamics and vibration reduction

Summer, 2011 - Magnetocaloric machine modeling and design

Edison Engineering Development Program, GE Appliances - Louisville, KY

January-June, 2012 - Front load clothes washer out-of-balance and load size detection

June-January, 2012 - Clothes dryer performance optimization

January-June, 2013 - Refrigeration sealed systems: Heat exchanger and capillary tube modeling and mixed refrigerant system design.

June-January, 2013 - Computer aided design team: Fluid and thermal system modeling and optimization.

January 2014 to present - Magnetocaloric team: System modeling and experimentation, heat transfer modeling and experimentation, machine design and optimization. 